\title{
COMBINING POSITRON EMISSION TOMOGRAPHY (PET) DATA WITH NEUROANATOMICAL CONSTRAINTS: COMPARING MODELS OF SINGLE-WORD PROCESSING
}

\author{
Erwin James Nikelski \\ Department of Psychology \\ McGill University, Montreal \\ December, 1996
}

\begin{abstract}
A Thesis submitted to the Faculty of
Graduate Studies and Research in partial

fulfillment of the requirements for the degree of Master of Arts
\end{abstract}

() Erwin J. Nikelski, 1996 
National Library of Canada

Acquisitions and Bibliographic Services

395 Wellington Street Ottawa ON K1A ON4 Canada
Bibliothèque nationale du Canada

Acquisitions et services bibliographiques

395, rue Wellington

Ottawa ON KIA ON4

Canada
The author has granted a nonexclusive licence allowing the National Library of Canada to reproduce, loan, distribute or sell copies of this thesis in microform, paper or electronic formats.
L'auteur a accordé une licence non exclusive permettant à la Bibliothèque nationale du Canada de reproduire, prêter, distribuer ou vendre des copies de cette thèse sous la forme de microfiche/film, de reproduction sur papier ou sur format électronique.

L'auteur conserve la propriété du droit d'auteur qui protège cette thèse. $\mathrm{Ni}$ la thèse ni des extraits substantiels de celle-ci ne doivent être imprimés ou autrement reproduits sans son autorisation. 


\section{Acknowledgments}

First and foremost, I would like to thank my advisors, Dr. Robert Zatorre and Dr. Laura Petitto for their guidance and encouragement. I would also like to express my gratitude to Dr. Denise Klein and Dr. Brenda Milner for allowing me to analyze two of their PET datasets.

I would also like to thank the staff of the Brain Imaging Center at the Montreal Neurological Institute for their kindness and assistance in helping me to better comprehend the complexities of PET data preprocessing. I also value the contribution of Mike Dama, who gave me a better understanding of structural equation modeling, in general, as well as the AMOS structural equation modeling software, in particular. The car rides with his dog were a great stress reliever. I also appreciate the assistance of Annie Le Bire and Patrick Bermudez for their help in translating the Abstract into French.

Special thanks go to Anat Geva for far-ranging and stimulating discussions, some of which were peripherally related to some of the issues addressed in this thesis, many of which were not. Thanks also go to Kelly Barlow for being a reliable source of coffee, an attribute that I value a great deal, and to Virginia Penhune for knowing who to ask to get an answer to absolutely any question.

Finally, I am thankful to Dr. M. P. Bryden for helping me to find my way, and giving me a gentle push to get me started.

This work was supported by a Natural Sciences and Engineering Research Council of Canada PGS-A scholarship, for which I am grateful. 


\begin{abstract}
The focus of this paper is to apply some of the new analytic techniques to positron emission tomography (PET) data in order to test the neuroanatomical plausibility of two very specific models of language processing: the model of Geschwind (1965), and that of Petersen et al. $(1988 ; 1989)$. Model plausibility was assessed by fitting both models to a word repetition and a synonym generation rCBF PET dataset using methods based on the examination of interregional correlations, and structural equation modeling.

Physiologically-based regions of interest were defined a priori through a review of PET activation literature. The current study demonstrated (1) the feasibility of using a priori defined physiological regions of interest in model evaluation, (2) the advantages of using structural equation modeling over interregional correlations, and (3) that neither model proved to be a particularly good fit to the data.
\end{abstract}


Résumé

Le but premier est de mettre en application de nouvelles techniques aux données de tomographie par émission de positrons (TEP) afin de démontrer la plausibilité de deux modèles très spécifiques d'assimilation du langage: le modèle de Geschwind (1965), et celui de Petersen et al (1988; 1989). La plausibilité des modèles à été évaluée en fittant les deux modèles à un dataset $\mathrm{rCBF}$ TEP de répétition de mots et de génération de synonymes en utilisant des méthodes basées sur l'examen de correlation interrégionale et le modelage d'équations structurées. Les régions d'intérêt à base physiologique ont été définies à priori à travers une revue de la littérature d'activation TEP. L'étude actuelle démontre (1) la faisabilité d'utiliser des régions d'intérêt physiologique définies à priori dans l'évaluation de modèles, (2) les avantages d'utiliser le modelage d'équations structurées aux correlations interrégionales, et (3) qu'aucun des deux modèles ne prouve à être un bon fit aux données. 


\section{Table of Contents}

Acknowledgments. $\mathrm{i}$

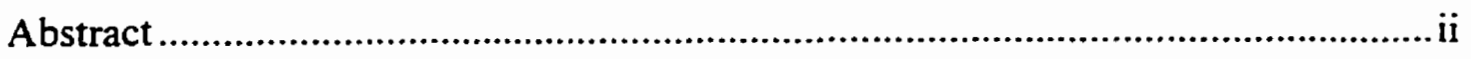

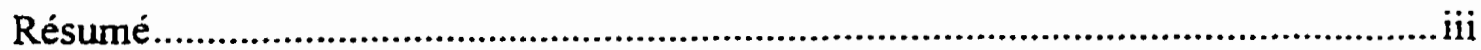

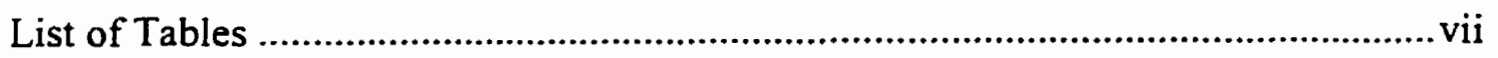

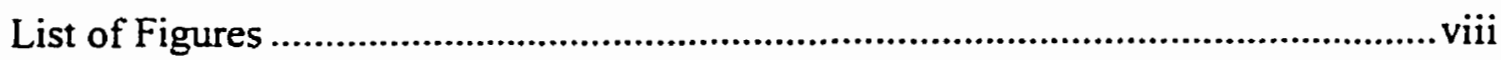

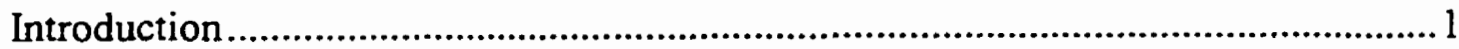

Positron Emission Tomography (PET) Data Analysis:

An Overview of Various Analytic Techniques ......................................2

Hierarchical Subtraction .....................................................2

Assessing Significant Change.................................................4

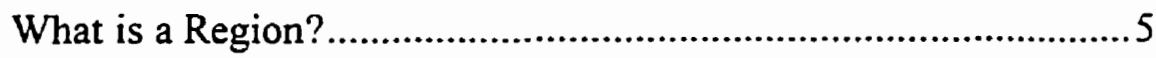

Association of Regions with other Regions ............................6

Neuroanatomical Regions Involved in Language ..................................9

Naming Neuroanatomical Regions ........................................9

Defining Functional Regions ................................................ 12

The Limbic System ........................................................... 15

Cingulate Gyrus (CG) ............................................ 15

The Frontal Lobe ................................................................21

Inferior Frontal Gyrus/Cortex - (IFG) ........................22

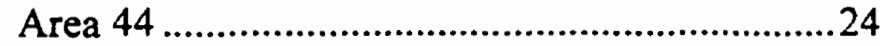

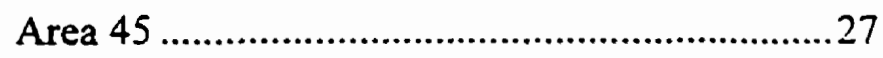

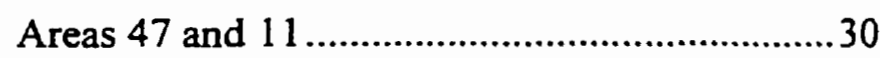

The Parietal Lobe.........................................................................31

Inferior Parietal Lobule (IPL): Supramarginal Gyrus.......32

Inferior Parietal Lobule (IPL): Angular Gyrus ................ 32

The Temporal Lobe ................................................................36

Superior Temporal Region (STR) ...............................36 
Primary Auditory Cortex (PAC).........................................37

Planum Temporale ........................................................... 37

Superior Temporal Gyrus (STG) .......................................39

Superior Temporal Sulcus (STS) .......................................43

Middle Temporal Gyrus (MTG) /

Temporo-Occipital Junction.........................................44

Models of Language Processing ..............................................................45

Serial Single-Route Model ............................................................46

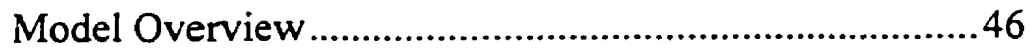

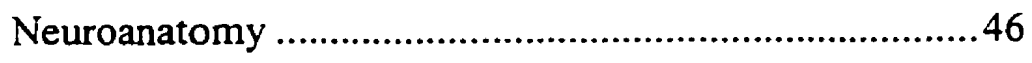

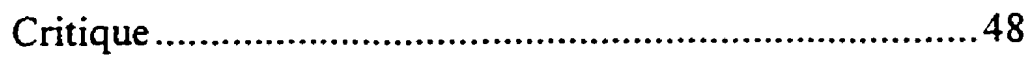

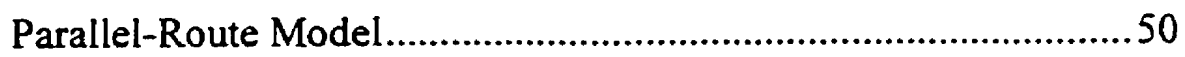

Model Overview ................................................................ 50

Neuroanatomy ....................................................................51

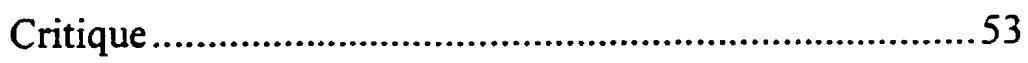

Using PET to Test Models of Single-Word Processing ................56

Experiment I: Assigning Substrate to Functional Processes........................................57

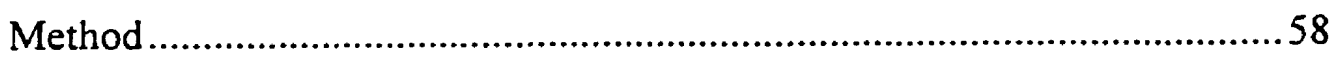

Criteria for Study Selection .......................................................5

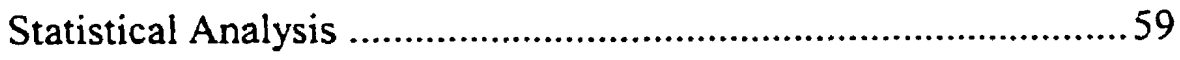

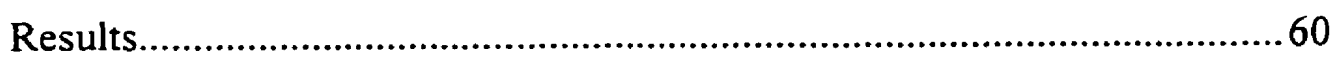

Frontal Lobe............................................................................62

Bilateral Clusters ...............................................................66

Unilateral Clusters ............................................................66

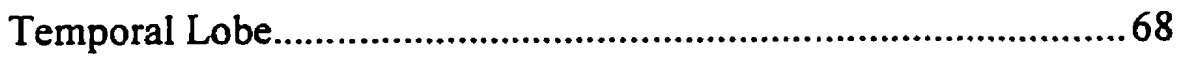

Bilateral Clusters .............................................................68

Unilateral Clusters ...............................................................69

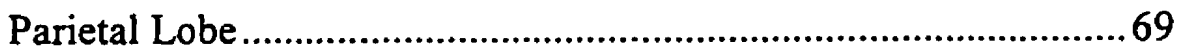

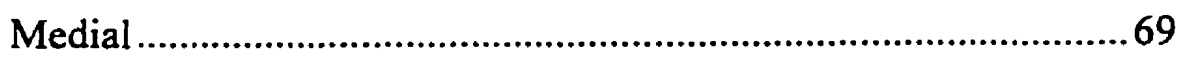

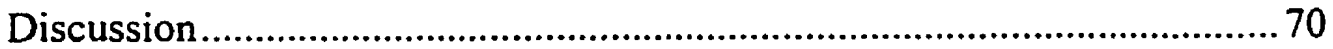


The Geschwind Model............................................................. 70

The Petersen et al. Model ..................................................... 72

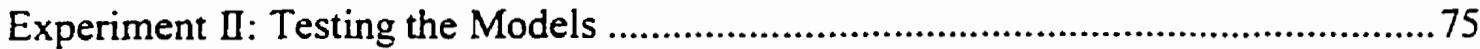

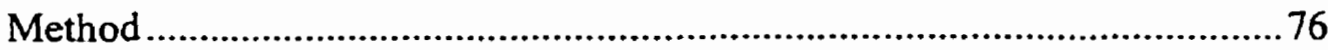

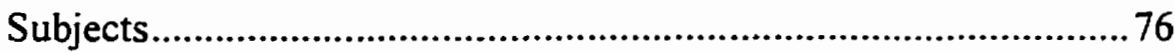

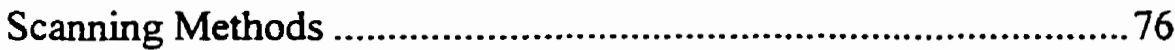

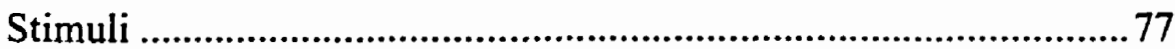

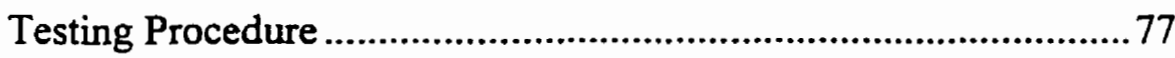

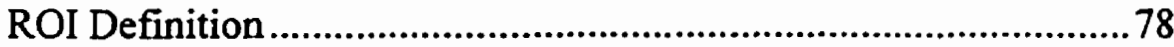

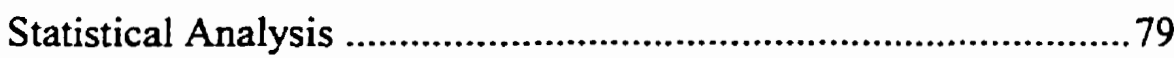

Interregional Correlations..........................................79

Structural Equation Modeling ...................................81

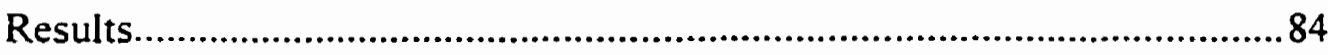

Interregional Correlations ....................................................8 84

The Geschwind Model ..............................................84

The Petersen et al. Model ........................................8 87

Structural Equation Modeling ..............................................8 87

The Geschwind Model .............................................88

The Petersen et al. Model ............................................91

Model Comparisons ..................................................94

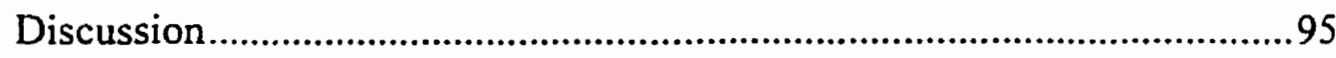

Interregional Correlations .......................................................95

Structural Equation Modeling ................................................. 97

Left vs. Right Hemisphere................................................... 100

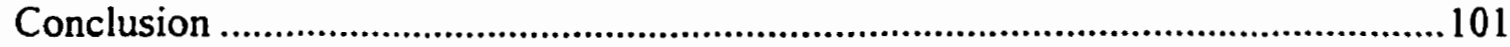

Future Directions ........................................................................ 104

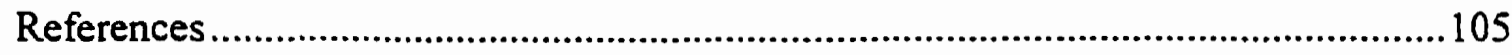

Appendix A: Significance Testing and the Correlation Coefficient ........................118 
Table 1: Modality and Processing Level Codes

Table 2: Task Specific Codes

Table 3: Classification Summary Information

Table 4: Cluster Information - Frontal and Temporal Structures

Table 5: Cluster Information - Parietal, Occipital, Medial, Cerebellar and Subcortical Structures .64

Table 6: Homologous Clusters.

Table 7: Inter-Cluster Distances Less than $20 \mathrm{~mm}$ .71

Table 8: Interregional Correlations for Repetition and Synonym Generation Conditions .85

Table 9: Differences in Interregional Correlations (Synonym Generation - Repetition) .86

Table 10: Significant Interregional Correlations Not Predicted by Either Model 


\section{List of Figures}

$\begin{array}{ll}\text { Page } & \end{array}$

Figure 1: The functional and anatomical connectivity of the cingulate gyrus.

Figure 2: The functional and anatomical connectivity of Brodmann's area 44.......26

Figure 3: The functional and anatomical connectivity of Brodmann's area 45.......29

Figure 4: The functional and anatomical connectivity of the angular gyrus............35

Figure 5: The functional and anatomical connectivity of the superior temporal gyrus.

Figure 6: The serial stage-based model of Geschwind (1965).

Figure 7: The parallel-route model of Petersen et al. $(1988,1989)$.

.52

Figure 8: Clusters, as determined by Experiment \# 1, superimposed upon a 305 brain aggregate.

Figure 9: Substrates assigned to the Geschwind (1965) model as a result of the cluster analysis.

Figure 10: Substrates assigned to the Petersen et al. $(1988,1989)$ model as a result of the cluster analysis.

Figure 11: Path coefficients assigned to the Geschwind (1965) model for both the repetition and synonym generation. .88 
Figure 12: Path coefficients assigned to the Petersen et al. $(1988,1989)$ model for the repetition condition

Figure 13: Path coefficients assigned to the Petersen et al. $(1988,1989)$ model for the synonym generation condition

Figure A-1: Stability of the bootstapped standard error of estimate of selected correlation coefficients as a function of the number of samples used in its calculation. 


\section{Introduction}

Serious study of the neural substrates involved in language processing began in the mid 19th century with the examination of aphasics, which allowed researchers to link symptomology observed prior to death with lesions found at post mortem. This technique led to findings of immense value with regard to substrates involved in language production (Broca, 1861; 1865), language comprehension (Wernicke, 1874), and reading and writing (Dejerine, 1891). Unfortunately, a backlash against these essentially localizationist findings in the early part of the 20th century effectively halted much of the research that was done in the area of the neuroanatomy of language, although clinicians continued to make use of the standard aphasia classifications as proposed by Lichtheim (1884). Study into the substrates associated with language was resurrected in large part due to the revival of the work of the classical authors by Geschwind in general, and in particular. by his disconnection theory (Geschwind, 1965). The cognitive revolution of the 1970's enabled researchers to study language processing by dissociating language processes from their substrate, thus proving to be a setback for study of the anatomical localization of language, but also allowing researchers to concentrate on language processing in normal non-lesioned, subjects. Today, a focus of research in the area of language processing by cognitive neuroscientists attempts to bring together cognitivelybased and neuropsychologically-based models of language processing with data provided from functional neuroimaging techniques, such as positron emission tomography (PET), as well as with new information with regard to anatomical connectivity in both humans and animals. This approach is, by definition, multidisciplinary.

In the following sections, the introduction will follow certain steps in order to explore how language processing, with emphasis on single-word processing, can be modeled using a systems approach. The first section will endeavor to give an appreciation for the capabilities of functional neuroimaging in general, and for the various analytical techniques that may be used to extract the information of interest, in particular. Next, the neuroanatomical and functional connectivity of regions that have been indicated as playing a significant role in language processing shall be explored. In particular, this section, rather than attempting to review all areas that might be implicated in language 
processes, will focus on the connectivity of neuroanatomical regions that serve a critical role in two specific models of language processing: (1) the model of Geschwind (1965), hereafter referred to as the Geschwind model, and (2) the model proposed by Petersen et al. $(1988 ; 1989)$, hereafter referred to as the Petersen et al. model. Anatomical connectivity shall be investigated via presentation of neuroanatomical data derived from the study of both human and non-human primates; functional connectivity via presentation of findings primarily derived from functional studies of humans. The final section will compare and contrast the Geschwind and Petersen et al. models with each other, as well as discuss the degree to which they reflect the neuroanatomical and functional findings presented in the previous section.

Positron Emission Tomography (PET) Data Analysis:

An Overview of Various Analytic Techniques

PET is a functional technique that makes it possible to investigate potential links between a given behaviour and the neural substrate associated with that behaviour. It is similar to other techniques, such as MRI, in that PET is capable of providing threedimensional images of the brain, albeit with a spatial resolution that is not quite as high as that typically available with MRI. Unlike MRI, PET requires the injection of a radioactively tagged marker. Functional activation sites are localized by detecting emitted radiation while the subject is asked to perform a task. Although $\mathrm{H}_{2}{ }^{15} \mathrm{O}$ is commonly used as a marker in studies of regional cerebral blood flow (rCBF), many different substances may serve as markers (e.g. fluorodeoxyglucose (FDG), neurotransmitters, etc.). Many different analysis techniques may be used in order to extract the desired information from the scanning dataset, with different techniques revealing different dimensions of the data. One of the most commonly used techniques with $\mathrm{rCBF}$ data is hierarchical subtraction.

\section{Hierarchical Subtraction}

The use of hierarchical subtraction was first introduced by Petersen et al. (1988, 1989). Studies of this type are generally designed as a hierarchy of tasks, with the baseline task being the least complex, and each subsequent activation task adding one clearly defined level of complexity to the task that immediately precedes it in the 
hierarchy. The correspondence between substrate and added behavioral complexity is gained by subtracting from the activations observed during a given task, the activations that occurred in the task immediately preceding it. The subtraction procedure works on a voxel-by-voxel level, in that the counts stored at voxel $x, y, z$ in the baseline volume is subtracted from the counts stored at the corresponding voxel location in the activation volume. This subtraction occurs over all of the voxels in the volumes, with the differences stored in a difference volume. Each voxel in the difference volume thus contains difference scores, reflecting both the positive and the negative differences in rCBF between the baseline and the activation condition, i.e. a large positive score reflects a large increase in $\mathrm{rCBF}$ in the activation condition as compared to the baseline condition, whereas a large negative score represents a large rCBF decrease.

Now, if one were to engage in a subtraction of a single baseline volume from a single activation volume, one would probably not see a series of discrete foci representing areas of blood flow increase or decrease, but rather, one would probably be faced with an image so highly bathed in noise, that only with great difficulty might one be able to identify any foci at all. This is due to the fact that changes in rCBF which result from the execution of a cognitive task are usually quite small, typically ranging from 3 to $6 \%$, and, as such, the signal is easily overwhelmed by unwanted noise. The strength of the signal when compared to the intensity of the noise is best quantified by a measure called the signal to noise ratio, in which a signal with a high signal to noise ratio is most easily detected. Thus, the problem of not being able to detect foci in a PET volume as a result of the signal being overwhelmed by noise is a consequence of having a low signal to noise ratio. The solution to the signal-to-noise problem was first systematically investigated by Fox et al. (1988), who found that substantial increases in signal-to-noise could be achieved by creating averaged volumes, reflecting activations across multiple performances on a given task, and then subtracting the averaged volumes. For example, one could have a given subject perform a baseline and activation task three times each, and then average all three baseline volumes and all three activation volumes prior to performing the subtraction. Unfortunately, relatively low sensitivity of the scanners available at the time made within- 
subject averaging impractical, and researchers therefore turned to the process of intersubject averaging, which, in turn, introduced yet another problem.

Averaging volumes across subjects introduces a number of problems, due to the fact that no two brains are geometrically alike. Brains can differ in three major respects: (1) orientation in space at the time of the scan, (2) size, and (3) shape. The universal solution to this problem has been to transform each brain into a standardized coordinate system prior to averaging. The transformation involves the application of a number of linear transformations which rescale and reposition each brain to match the size and position of a standardized brain. The standardized space most frequently used is that of Talairach and Tournoux (1988). The transformation of volumes from native space, while having the advantage of allowing averaging across subjects and thus increasing the detectability of foci by increasing the signal-to-noise ratio, does, however, come at the price of decreased spatial resolution. The decrease in spatial resolution is introduced at image reconstruction by smoothing each volume via a reconstruction filter, which is necessary in order to account for between-subject differences in gyral anatomy. Any increase in signal-to-noise ratio must thus always come at the expense of decreased spatial resolution.

\section{Assessing Significant Change}

Once the signal-to-noise ratio has been increased via inter-subject averaging of volumes prior to subtraction, one is now in a position to display the resultant averaged subtraction volume and identify areas of $\mathrm{rCBF}$ increase or decrease related to the additional load associated with the activation task being studied. Although visual inspection of this volume can provide converging evidence with regard to localization of function, much would be gained by being able to objectively quantify and assess the significance of local minima and maxima. Fox et al. (1988) attempted to provide this needed level of quantification with their technique of change-distribution analysis. Change-distribution analysis uses an algorithm to search the inter-subject averaged difference volume for local minima and maxima via a two stage process. Firstly, the algorithm defines local minima and maxima to occur at all those voxels at which the mean $\mathrm{rCBF}$ change is greater than that of all $\mathrm{N}$ surrounding voxels, where $\mathrm{N}$ is a function 
of the width of the reconstruction filter used. Secondly, a center-of-mass algorithm, operating over a spherical region of interest (ROI) whose size is determined by the size of the reconstruction filter, is used to find the center of the potential activation focus. This procedure generates a list of ROIs, each identified by an $x, y, z$ coordinate and a magnitude. The degree of data reduction accomplished through the use of this procedure is demonstrated in that an average volume consisting of approximately 50,000 intracranial voxels can typically be reduced to between $350-700$ foci. This procedure also has the advantage of allowing for the calculation of an omnibus test statistic, thus enabling the researcher to state whether the conditions being compared actually did significantly differ in $\mathrm{rCBF}$.

An alternate method of assessing significance requires the creation of a statistical parametric map (SPM). An SPM is a three-dimensional volume similar to the difference volume discussed previously. The major difference is that each voxel, rather than containing a magnitude, contains a test statistic instead. The primary advantage of this over change-distribution analysis is that each voxel can be assigned a significance value. Assigning significance at the level of the voxel allows for testing much more complex hypotheses (see Friston et al. (1991) for a complete discussion and Worsley et al. (1992) for a more theoretical presentation on specific methods). For example, whereas change distribution analysis only allows for a test of the omnibus hypothesis, i.e. "Was there any significant change at all?", SPMs make it possible to test the following three types of hypotheses: (1) a test of significance at a particular predefined location, i.e., "Was there significant change at voxel $x, y, z$ ?", (2) a test of the omnibus hypothesis, i.e., "Was there any significant change at all?", and (3) a test of significance over all voxels, i.e., "Which pixels were significant at $\mathrm{p}=.05$ ". The increased power of SPMs, as defined by being able to identify the significance of a blood flow or metabolic increase or decrease, has resulted in the predominance of this method of significance assessment.

\section{What is a Region?}

Many of the techniques to follow, require that the analysis be done at the level of a region of interest (ROI). This requires us to first discuss exactly how ROIs are defined, since differently defined ROIs will undoubtedly impact the results of the analysis. 
Basically, there are two primary ways of defining ROIs: (1) anatomical, and (2) functional. Anatomical ROI definition, is an older technique which is currently the method of choice in studies of cerebral glucose metabolism (Horwitz et al., 1984, Horwitz et al., 1986; Azari et al., 1992). This technique typically uses a template to divide the brain a priori into a number of anatomically based ROIs. In the study by Horwitz et al. (1988), the brain was divided into 28 bilaterally defined ROIs and 3 midline ROIs, giving a total of 59 regions of interest. Voxel activation for all voxels contained within each ROI are subsequently averaged in order to provide a measure of mean ROI activation strength. Although this technique does result in a drastic reduction in data and, as such, aids in the interpretability of results, it is not without drawbacks. For example, the decision to sample some regions by declaring them to be of interest, while ignoring other areas, may introduce a selection bias. The effects of this selection bias can be attenuated or eliminated by using large ROIs, thus effectively allowing the entire brain to be sampled. Unfortunately, this solution to the selection bias problem introduces a somewhat different problem, since spatial resolution suffers as ROI size increases (see Fox (1991) for an in depth review). In contrast to the anatomical method, and in response to some of the problems highlighted by Fox (1991), the functional method defines ROIs a posteriori, i.e., after the data have been collected. An example of a technique which uses functional ROI definition is change-distribution analysis (Fox et al., 1988). As previously discussed, foci are identified through an examination of the local minima and maxima contained within the subtraction volume. Once identified, the voxel is then made the center of a spherical ROI. Although this method has a number of advantages over the anatomically based method, functional ROI definition is still used primarily in studies of cerebral blood flow.

\section{Association of Regions with Other Regions}

Another area of interest is the potential functional and / or anatomical connections that might exist between various different regions. That is to say, although it may well be of interest to be able to say that a synonym generation task results in the activation of a region located at the medial superior temporal gyrus, it would also be of great interest if one would be able to identify other regions, whose degree of activation varied in 
conjunction with that of the medial superior temporal gyrus. Regions whose degree of activation is correlated, might allow one to postulate the existence of either an anatomical or a functional pathway connecting the structures. A very common way to investigate possible associations between various areas is to create a correlation matrix of all of the ROIs, correlated across subjects. Horwitz et al. (1988), for example, used interregional correlations to investigate how autism might affect regional cerebral metabolic rates ( $\mathrm{gCMR}$ ). ROIs were anatomically defined (28 bilateral, 3 medial), and then intercorrelated with each other, across two separate groups of 14 subjects (one control group, one autistic group). The resultant two intercorrelation matrices were then subtracted from each other, creating a difference matrix. Analyses were done at both the level of the individual ROIs, as well as at the level of the lobular anatomy, made possible by grouping the ROIs. Analysis of the difference matrix enabled the authors to determine in which correlations the two groups most differed, e.g. (1) the autistics had fewer large positive correlations between pairs of regions in the frontal and parietal lobes, (2) the size of the correlations between the frontal/parietal regions and the thalamic nuclei was decreased in the autistic group, and (3) $70 \%$ of the correlations were lower in the autistic group.

The previously discussed study should serve to highlight a characteristic of most PET studies: a very small number of subjects. This is particularly problematic if one attempts to do correlations. since correlations become increasingly unstable as the number of subjects over which one correlates decreases. The previous study generated two correlation matrices, each created by correlating across only 14 subjects. Fortunately, the validity of the findings of this study are not in doubt, since the authors applied special procedures to help ensure the reliability of the correlation coefficients. The two procedures that were applied are called "bootstrap" and "jackknife" (Efron, 1981). The purpose of the jackknife procedure is to eliminate any correlation that was significant merely due to a single outlier. It works by recomputing the partial correlation coefficient with each subject, in turn, removed from the sample. Any correlation that has a large value due only to one particular subject, will show a large drop in the correlation when that subject is removed from the sample. The bootstrap procedure serves to eliminate 
correlation coefficients with large variances. This procedure works by randomly selecting, with replacement, $\mathrm{N}$ correlations from a sample of $\mathrm{N}$, and then computing the partial correlation coefficient. When this has been done $\mathrm{N}$ times, the partial correlation coefficients are examined and a decision is made about the stability of the correlation. One typically will only regard a correlation coefficient as stable if the partial correlation coefficients exceed 0.5 on $\mathrm{N}-1$ of $\mathrm{N}$ bootstraps. A similar procedure can also be applied to the estimation of standard error for each correlation.

Alternatively, it is also possible to compare the degree of association between regions on a voxel-by-voxel basis (Friston et al., 1992a; Zeki et al., 1991). Zeki et al. (1991) used this type of approach in attempting to localize areas functionally associated with area V4 (colour detection). This was accomplished via the following steps: (1) generate a difference volume by subtracting a baseline condition from an activation condition which would cause area V4 to be stimulated, (2) find area V4 in the subtraction volume and choose a "reference" voxel from within it, (3) create a covariance matrix by calculating, across subjects, how every other voxel covaries with the reference voxel, and (4) analyze the covariance matrix in order to determine which area(s) covaried with the area containing the reference voxel. This method has an advantage over the previous method in that the analysis is performed at the level of the voxel rather than at the level of an ROI, thus potentially providing much better spatial resolution, limited, of course, by the degree of spatial smoothing applied during image reconstruction..

A third method of assessing the degree of association between regions makes use of structural equation modeling (Horwitz \& McIntosh, 1993; McIntosh \& Gonzalez-Lima, $1992 ; 1993 ; 1994 ;$ McIntosh et al., 1994). The appeal of this method is that it allows functional interactions to be viewed in the context of a network of inter-associated regions, rather than simply as a series of pairwise comparisons. Although the use of structural equation modeling has long been applied in the social sciences, it is still in its infancy with regard to the analysis of functional data. The first step in the use of this technique is to create an anatomical network model of the system whose functional interactions are to be modeled. This will usually involve defining which anatomical areas are assumed to be of potential significance, the anatomical links which are known to exist 
between these areas, as well as the direction of these anatomical links. For example, one may be interested in investigating the functional associations contained within a network comprised of areas A, B, and C. Not only must one define that area A is linked to area B, and that area $B$ is linked to area $C$, but one must also state that area $A$ is linked to $B$ via a bi-directional link, whereas area $B$ is linked to $C$ via one link going from $C$ to $B$. After the anatomical network has been defined, the next step is to use the functional data (blood flow or metabolic) to create an intercorrelation matrix using the ROIs defined in the anatomical network. Once the pairwise correlation coefficients have been obtained, the final step is to combine the information contained in the anatomical network model with the functionally derived information, via the use of a specialized software package such as LISREL (Jöreskog and Sörbom, 1989), EQS (Bentler, 1985) or AMOS (Arbuckle, 1992), to create a functional network model. The functional network model effectively represents all the influences of the regions contained within the model on each other, constrained, however, by the anatomical connections defined within the anatomical network model (see McIntosh \& Gonzalez-Lima (1994) for a complete discussion). Once the functional network model has been generated, inter-regional associations can be assessed by examining the path coefficients assigned to each anatomical link. Based upon this information, the researcher may, in an attempt to fine tune the anatomical model, wish to exclude previously defined regions from or include new regions into the anatomical model and then regenerate the functional model from the revised anatomical model.

\section{Neuroanatomical Regions Involved in Language}

Assigning neuroanatomical substrate to a model generally involves defining the actual areas involved in the function of interest, such as language processing, and then defining the connectivity between those areas. Although this appears quite straightforward, the seemingly simple process of naming a specific neuroanatomical region can be a source of confusion.

\section{Naming Neuroanatomical Regions}

Naming the various neuroanatomical areas that may make a contribution to language processing is not an easy task. Somewhat surprisingly some of the problems arise from 
the simple fact that different areas of investigation, having different goals, have developed different naming conventions. Different regions of neocortex, for example, have been labeled according to cytoarchitecture (Brodmann, 1909), order of myelinization of axons (Flechsig, 1920), a standardized stereotaxic space (Talairach \& Tournoux, 1988), functionally defined processing modules, and gyral anatomy. As such, we find ourselves in a situation in which, for example, the primary cortical receiving area for audition can be defined as: (1) the primary auditory cortex, (2) area A I, (3) area 41 (Brodmann, 1909), (4) Heschl's gyrus, and (5) approximately located at coordinate [45, 24,16 ] (Talairach \& Tournoux, 1988). This is further complicated by the fact that the above mentioned maps do not only differ in that the defined areas are differently named, but more importantly, in that there may be no real correspondence between regions defined by the various maps. As such, translating from one system to another may either be impossible, or may require the application of some rather inspired assumptions. In addition to the above problems comes the fact that much of the clinically-derived aphasia literature has historically enjoyed reporting their findings based on pseudoneuroanatomical areas, such as Broca's or Wernicke's areas, whose actual boundaries are still a matter of some debate.

The above mentioned problems, though not insurmountable, do require us to impose some constraints and make some observations. Firstly, studies that have reported substrate using a nomenclature that only allows for a very general localization (e.g. Broca's area) will only be of use to investigators who are interested in making very general claims. Studies that aim to make more specific claims with regard to language localization, such as this one, need to make use of a nomenclature that allows for this level of specificity. It is for this reason, among others, that many studies have chosen to report their findings using cytoarchitectonic parcellation and/or gyral anatomy. This study will follow this course as well. Secondly, although many studies interested in functional-anatomical correlations report their findings using cytoarchitectonic maps, many clinical studies as well as studies of anatomical connectivity report their findings using gyral anatomy. It therefore becomes necessary to be familiar with this nomenclature as well. As an aid to comprehension, this paper will occasionally give both 
cytoarchitectonic and anatomical labels for a given region, although focus will remain on using cytoarchitectonic designations whenever possible.

Defining neuroanatomical regions via cytoarchitecture is preferred by many researchers due to the fact that (1) many studies have demonstrated a correlation between laminar organization and function, e.g. the clear difference in the laminar organization of primary receiving cortex when compared to that of adjacent association cortex, (2) a given area is more likely to send projections to another area if the receiving area has a similar laminar organization, thus allowing researchers to make inferences about connectivity, and (3) gyral anatomy quite often reflects cytoarchitectonic boundaries, since many (but not all) cytoarchitectonic boundaries are located in sulci, known as "limiting sulci" (see Rademacher et al., 1993 for an in depth discussion). As such, much work has been done with regard to better defining the cytoarchitecture of the cortex in human and non-human primates. Unfortunately, the detection and delimiting of subtle changes in laminar organization, as well as the naming of those areas, involves a certain degree of subjective judgment, and it is therefore not surprising that researchers have proposed a number of slightly different cytoarchitectonic maps. Thus, the most commonly used human-based brain atlas, that of Brodmann (1909), uses numbers to designate areas, whereas the atlas of von Economo and Koskinas (1925) uses a naming system based on alphabetic labels. Happily, the isolated cytoarchitectonic regions are quite similar, and it is thus not unduly difficult to translate from one area name to another. The same sort of dilemma exists for monkey-based brain atlases. The greatest problem, however, arises when one wishes to take a monkey-based area and find its homologous region in the human brain. This ability is of great utility since much more work has been done in the area of mapping function to anatomy and physiology in the monkey than in the human. A step towards being able to integrate human and monkey findings has resulted from the work of Petrides and Pandya (1994). The authors created cytoarchitectonic maps of the prefrontal regions of both monkey and human brains, and then noted areas of similarity. Most of the anatomical and physiological information to follow, detailing the structure of the brain will have been done primarily on rhesus monkeys. Prefrontal areas shall be converted to their human atlas equivalent (Brodmann, 
1909) using the tables provided by Petrides and Pandya (1994). Unfortunately, other areas present a bit of a problem, since an equivalent inter-species cross-index does not yet exist. As such, areas will assume to be similar across species if (1) the area in question is part of a primary sensory or parasensory area, since one would expect less inter-species variation at that level, or (2) if the known cytoarchitecture is similar between the two species.

On a point of accuracy, it should be noted at that, although activations, as reported from functional studies, are reported with a great deal of specificity, e.g. BA 44 or BA 45 , it should be remembered that functional imaging studies that use inter-subject averaging, as most of them do, by their very nature must sacrifice some degree of spatial specificity. Steinmetz and Seitz (1991) found that brains transformed into a given stereotaxic space show residual zones of variation of between 1.5 to $2 \mathrm{~cm}$ along defining sulci such as (1) the anterior ascending ramus, which defines the boundary between BA 44 and BA 45, (2) the Sylvian fissure, and (3) the posterior ascending ramus, which serves to define the supramarginal gyrus and thus, BA 40 . Nonetheless, it would seem prudent to retain the degree of specificity reported in the original studies, rather than simply attempting to group activations into some aggregate region (e.g. Broca's area), since such an aggregation would necessarily result in the loss of potentially crucial information. Spatial information must, however, be interpreted with care, and as such some activations that fall very close to a cytoarchitectonic boundary have been noted. Thus designations such as $44 / 45$ or $44 / 6$ (which are quite commonly observed) serve to reflect the fact that the observed activations occurred close to a cytoarchitectonic boundary.

\section{Defining Functional Regions}

The areas involved in language processing can be identified functionally via techniques such as PET, or clinically via the study of lesion to symptom correlations. Although both of these techniques have advantages and disadvantages, the disadvantages associated with the use of clinical data are of such importance, that we shall now briefly turn to the problems associated with the use of clinically-derived information as a source of information about language processing. 
Historically, language substrates have been inferred as a result of the post mortem study of aphasics. Although this approach has provided the field with valuable information over a great many years, this approach, unfortunately, does have a number of disadvantages. Firstly, one is relegated to the study of language in an abnormal population, i.e. a population in whom language is not functioning properly. This raises questions about the generalizability of findings to a normal population, since it is well known that cerebral reorganization can occur following trauma (Rasmussen \& Milner, 1977). Secondly, localization of a particular lesion does not necesarily mean that the lesioned area is critical, or even involved, in language processing. A lesion might cause a language disturbance by interrupting the connections between other, non-lesioned, areas. Thirdly, lesion data, at best, only gives information about areas that are both critical and spatially focal, i.e. not distributed. That is to say, any area that might have a redundant processor, or that might have its processing distributed over a larger region of cortex, might not result in any noticeable language impairment as the result of a lesion. Finally, most lesions are the result of either hemorrhage or ischemia. These lesions can cause extensive damage to a great number of cortical, subcortical, and white matter structures, thus localizing of which of the damaged areas actually contributed most to language processing becomes impossible. The effects of this problem can be seen in that the defined extent of any given language-significant area can become so large and poorly defined as to be of no use with regard to linking anatomy to behaviour. In this regard, some aphasia researchers (Schwartz, 1984; Caramazza, 1984) even argue that the correspondence between syndromes such as Broca's aphasia or Wernicke's aphasia and any particular substrate is so poorly defined, that any studies that categorize their subjects by aphasia syndrome, expecting the syndrome to be an indirect measure of the locus of damage, are fatally flawed. The above having been said, it should be emphasized that the above mentioned problems do not attempt to minimize the great contribution that classical aphasiology has made to the study of the neural substrates of language. They merely serve to highlight that the traditional lesion-based approach is not without its problems, and that other techniques, such as PET, have gained tremendously in recent years precisely due to their ability to avoid the aforementioned problems. 
The following sections will present findings obtained using a number of different techniques; however, since a major focus of this paper is the integration of neuroanatomical findings with those derived from PET, the emphasis will be placed on presenting functional rather than clinical data. Although the neuroanatomical connectivity of areas that are implicated in playing a role in language-related processing shall be presented in the text to follow, as well as often summarized via figures, it should be noted that an exhaustive presentation of all known neuroanatomical linkages are beyond the scope of this paper. Since the figures will serve to incorporate both neuroanatomical and functional findings in a condensed form, a brief word is required to explain the labeling conventions used. Firstly, connectivity between two neuroanatomical areas is depicted via a solid line connecting those two areas. If, in addition, the directionality of the connection is also known, this shall be indicated via an arrowhead; strong reciprocal connections shall be indicated by a connecting line with two arrowheads. Further information about neuroanatomical connectivity may also be given by naming the neurotransmitter known to be present in a given linkage, as well as its action, inhibitory or excitatory, if known.

Functional connectivity, i.e. presumed connectivity based upon functional data can be of two types: (1) CoAct (+/-) indicates that functional studies have found these areas to coactivate either in a positive direction, i.e. they both activate at the same time, or in a negative direction, i.e. when one area activates, the other usually deactivates, and (2) Corr $(+/-)$ indicates that activity within two areas has been found to correlate in either a positive or a negative direction. CoAct findings, the most common type, result from subtractive techniques, whereas Corr findings are the result of performing inter-regional correlations. Finally, the figures attempt to correlate specific substrates with specific tasks by noting which tasks resulted in substrate activations or deactivations. To be able to represent this information on a figure, activations were classified along three dimensions: (1) input modality of the stimulus, (2) primary processing level involved in carrying out the task, and (3) a task specific description. For example, an activation described as being $A W R p$ was the result of an auditory (A) word (W) repetition (Rp) 
task. The single-letter codes used to represent input modality and processing level information are given in Table 1; the 2-letter task specific codes are described in Table 2.

Insert Table 1 about here

Insert Table 2 about here

Although many regions of the brain have been implicated in language processing, we are obviously unable to examine them all. As such, the following section shall be limited primarily to a discussion of those regions that play a role in either the Geschwind or the Petersen et al. models. The anatomical and functional findings presented in the following section should enable us to provide a better critique these two competing models.

\section{The Limbic System}

The limbic system, itself, is not known to make any direct contribution to either the comprehension or the production of language. However, due to the vast degree of connectivity between the limbic system and virtually every other region of the brain (with particular emphasis on the reciprocal connections with prefrontal regions), components of the limbic system may have profound indirect effects on language. This is evidenced by the fact that, as shall be detailed below, activation of the anterior cingulate and other limbic components is commonplace in PET language studies. Having said that, the literature on the connections of the limbic system is almost without end. As such, I will necessarily be forced to limit the description of limbic system connectivity to a minimum, while focusing mostly on PET studies which have resulted in limbic system activation. Cingulate Gyrus (CG)

The bulk of cingulate activations have been found to occur in the anteriormost section of the CG. The exact nature of the contribution to language processing made by 
Table 1

Modality and Processing Level Codes

\begin{tabular}{cll}
\hline & Description & Example \\
\hline Input Modality & & \\
X & N/A & \\
V & visual & \\
A & auditory & \\
Processing Level & & \\
N & noise & \\
S & pseudowords & "tweal" \\
W & words & "tree" \\
L & letter & "lrdttrx" \\
O & other & \\
\hline
\end{tabular}


Table 2

Task Specific Codes

\begin{tabular}{|c|c|c|}
\hline & Description & Example \\
\hline \multicolumn{3}{|l|}{ Task } \\
\hline Ld & lexical decision & make a word/nonword decision \\
\hline Wg & word generation & generate a word (any word) with a given attribute \\
\hline $\mathrm{Vg}$ & verb generation & generate one verb for a given noun \\
\hline $\mathrm{Sg}$ & synonym generation & $\begin{array}{l}\text { generate one synonym for a given } \\
\text { adjective/adverb }\end{array}$ \\
\hline $\mathrm{Ag}$ & antonym generation & generate one antonym \\
\hline Fw & word fluency & generate as many four letter words as possible \\
\hline $\mathrm{Fc}$ & category fluency & $\begin{array}{l}\text { generate the names of as many animals as } \\
\text { possible }\end{array}$ \\
\hline $\mathrm{Fv}$ & verb generation fluency & $\begin{array}{l}\text { generate as many verbs as possible for a given } \\
\text { noun }\end{array}$ \\
\hline Fo & orthographic fluency & generate words beginning with the letter "a" \\
\hline $\mathrm{Rd}$ & read & read a word or pseudoword \\
\hline $\mathrm{Rp}$ & repeat & repeat a word, pseudoword, or letter \\
\hline Ps & passive & listen/look passively \\
\hline Sc & semantic categorization & $\begin{array}{l}\text { Compare } 2 \text { words for semantic features, ideally } \\
\text { this task will have no STM component }\end{array}$ \\
\hline $\mathrm{Sm}$ & semantic monitoring & $\begin{array}{l}\text { monitor input for a word with a given semantic } \\
\text { attribute. This task will require the subject to } \\
\text { retain a target attribute in memory }\end{array}$ \\
\hline $\mathrm{Pd}$ & phonemic discrimination & $\begin{array}{l}\text { compare two phonemes and make a } \\
\text { same/different judgment }\end{array}$ \\
\hline $\mathrm{Pm}$ & phonemic monitoring & $\begin{array}{l}\text { monitor words/pseudowords for a given target } \\
\text { phoneme }\end{array}$ \\
\hline $\mathrm{Fd}$ & feature discrimination & identify a visual feature \\
\hline Ry & rhyming judgment & $\begin{array}{l}\text { decide whether one word/pseudoword rhymes } \\
\text { with another }\end{array}$ \\
\hline
\end{tabular}


the anterior $C G$ is still unclear, although it has been implicated as taking part in a number of different systems. Some have proposed that the anterior CG in an important component of an anterior attentional system, which is primarily concerned with the selection among competing, complex contingencies, i.e. target detection or "selection for action" (Posner et al, 1988; Petersen et al., 1988; 1989; Posner \& Petersen, 1990). A somewhat different view of the role of the anterior cingulate is held by Fletcher et al. (1995), who suggest that the anterior cingulate may play a role in the maintenance of goal-directed behaviours, in particular, they propose that it may be involved in the suppression of other influences that might adversely affect goal attainment. Friston et al. (1993) see the anterior CG involved in two independent functional systems, (1) an attentional system, and (2) an intentional system. According to the authors, activation of the proposed intentional system, which is critical to the intrinsic generation of words, was found to be invariant over time, whereas activation of the attentional system, critical for the acquisition of perceptual set, was found to decrease monotonically over time, i.e. level of activation decreased with practice. They emphasize the finding that any given anterior cingulate activation may reflect the operation of two independent and overlapping anatomical systems.

Pandya et al. (1981) divide the CG into three sections, based on the connectivity of the various sections. The rostral portion (BA 25, BA 32) has connections to the frontal convexity (BA 9, BA 11/12/13) as well as the rostral STG. The medial cingulate (BA 24) receives afferents from various thalamic nuclei, and projects efferents to frontal premotor (BA 6), frontal eye field (BA 8), and orbital frontal cortex (BA 11/12). The caudal cingulate (BA 23) sends efferent projections to BA 9 (DLPFC) and BA 11/12 (orbital frontal cortex). Goldman-Rakic (1987) somewhat simplified this scheme by noting that rostral efferents (BA 24) differ in connectivity from caudal efferents (BA 23), thus pointing to a potential rostral-caudal difference in cingulate function. The anterior CG has strong reciprocal connections to the prefrontal cortex (Goldman-Rakic, 1987), and in particular to BA 6, BA 9, BA 12, and dorsal BA 8. A study of the cytoarchitecture of the rostral CG found it to be agranular and have a prominent layer V (Vogt et al., 1995). The caudal $C G$, which appears to be less involved in language processing, projects to areas 9 , 
11 , and 46 (Pandya et al., 1988) among others. The retrosplenial area lies somewhat caudal to the caudal CG, and can be argued to be a part of the parahippocampal formation, although its connectivity shall briefly be discussed here, due to its proximity to the CG. The retrosplenial area is comprised of areas BA 26, BA 29, and BA 30. In a study which aimed to describe the connections between the DLPFC and the hippocampal formation. Goldman-Rakic et al. (1984) discovered a lateral and a medial pathway connecting these two structures. Of interest is the fact that the medial pathway is a major source of reciprocal connections between the DLPFC (BA 9 in the monkey) and the presubiculum (the retrosplenial cortex). Moreover, the presubiculum also receives afferents from temporal and parietal association cortices, and sends efferents to the entorhinal cortex. Thus the presubiculum may serve as a link through which multimodal association areas may merge inputs and gain access to the mesial memory system; a system which would be of obvious importance with regard to the encoding, but not the retrieval, of language. An examination of Figure 1 appears to support the above presented neuroanatomical evidence of viewing the $C G$ as being comprised of an anteromedial section, which activates frequently during language tasks, a caudal section, which appears less involved in language-related processing, and the retrosplenial area, which is primarily involved in episodic memory encoding (Fletcher et al., 1995).

\section{Insert Figure 1 about here}

A detailed examination of anterior cingulate activations shows most of them to involve both auditory and visual presentation of words, with most tasks requiring semantic processing such as verb fluency (Warburton et al., 1996), category fluency (Frith et al., 1991 a; Warburton et al., 1996), orthographic fluency, (Frith et al., 1991a), verb generation (Petersen et al., 1989; Raichle et al., 1994), word generation (Friston et al., 1993), synonym generation (Klein et al., 1995), semantic monitoring (Petersen et al., 1988; 1989), semantic categorization (Fletcher et al., 1995; Zatorre et al., 1996), episodic 


\section{Comparing Models}

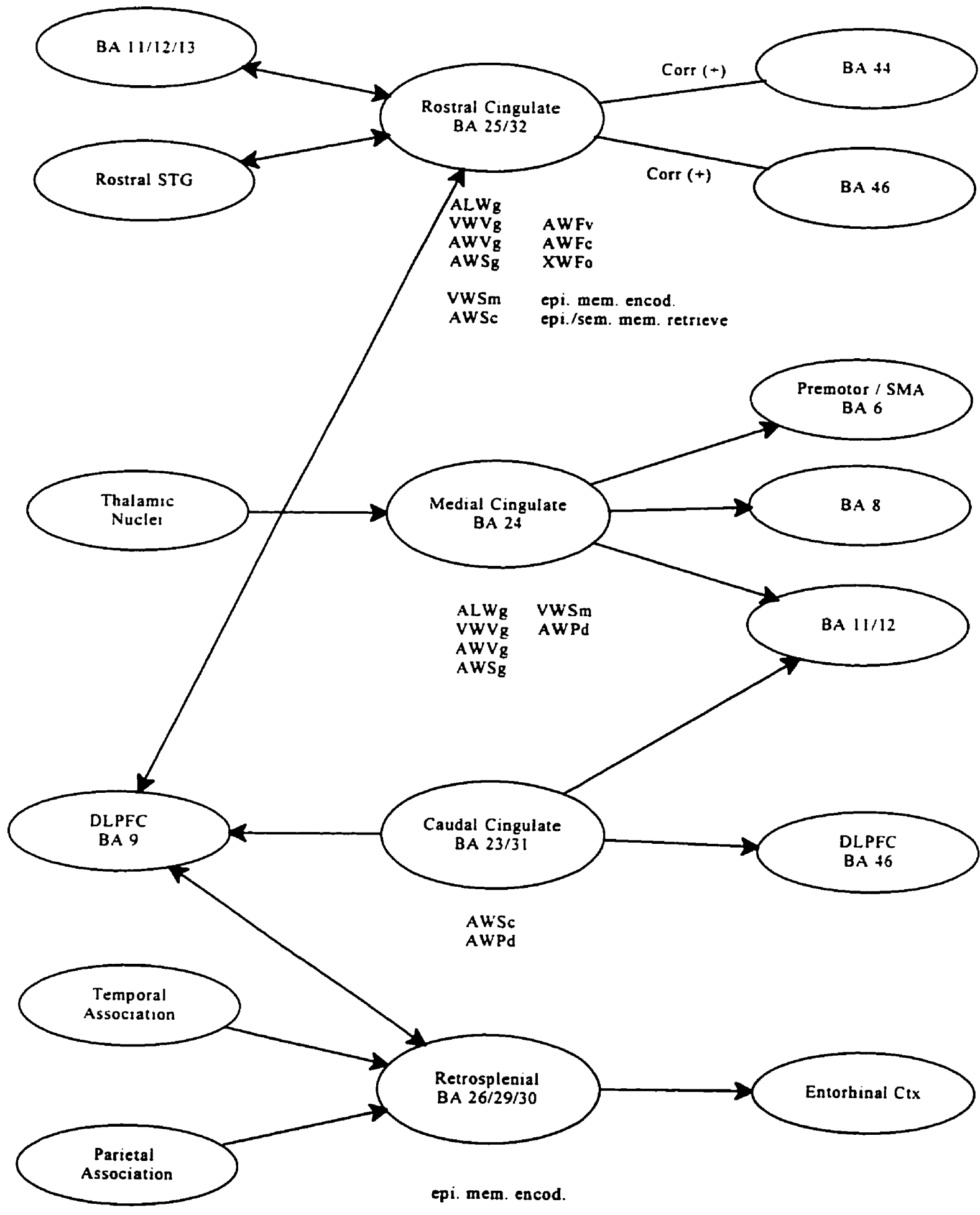

Figure 1 
memory encoding (Fletcher et al., 1995), and episodic and semantic memory retrieval (Fletcher et al., 1995). Only one phonological task, a phonemic discrimination task (Zatorre et al., 1992), resulted in anterior cingulate activation.

Relatively few caudal cingulate activations were noted, with the only common attribute being that all of the tasks involved the auditory presentation of words. Activations were noted as a result of phonemic discrimination (Zatorre et al., 1992; 1996), and semantic categorization (Demonet et al., 1992; Zatorre et al., 1996) tasks. Similarly, activation of the retrosplenial area also occurred only rarely, in response to an episodic memory encoding task (Fletcher et al., 1995).

\section{The Frontal Lobe}

Recent work on the anatomical connectivity and functional significance of frontal cortex has implicated it in functions that are primarily supramodal and integrative in nature. Common to many theories of frontal lobe function, is its proposed involvement in the temporal organization of potentially complex behaviours, although many researchers also agree with the position of Goldman-Rakic (1987), who views the frontal cortex as significantly contributing to behaviours that are not externally triggered. Work with nonhuman primates has been then the primary source of information with regard to obtaining a better understanding of frontal cortical function and connectivity, and this approach has been quite effective. Unfortunately, this focus on animal work, has made it exceedingly difficult for researchers to develop a general framework defining possible frontal contributions to an ability that is distinct to humans, i.e. language. An interesting framework has, however, been proposed by Alexander et al. (1989), the result of an integration of non-human primate findings on neuroanatomical connectivity and human lesion studies. This framework separates posterior/basal systems, which are capable of some independent operation, from frontal functional systems, which exercise control on the posterior/basal systems. The authors identified four frontal functional systems: (1) a system involved in motor control (BA 4, BA 6), responsible for articulation and linguistic prosody, lateralized to the left posterolateral cortex, including various subcortical components, (2) a cognitive system (BA 44, BA 46, BA 6), lateralized to the left lateral frontal cortex, and involved in grammar and word-finding, (3) a formulation system, 
located in anterior frontal regions, implicated in more abstract verbal skills, and (4) an activation system, located in medial frontal cortex (SMA, anterior cingulate), and involved in the initiation of verbal communication. The systems vary in their degrees of lateralization, with the motor and cognitive systems being more lateralized than either the formulation or the activation systems. Although this framework benefits from the fact that it was constructed based upon current studies of neuroanatomical connectivity, it also does have a few potential drawbacks. Firstly, the flexibility of the framework is a result of a lack of specificity with regard to the precise definition of the substrates involved in, and the postulated functions attributed to, each system. Secondly, validation for the functions assigned to each systems, has come primarily through the examination of damaged language systems, i.e. patient populations.

Innumerable connections exist between the frontal lobes and other brain regions, most of which are reciprocal in nature. Neurotransmitter distribution of the frontal cortex is still under investigation. Cholinergic neurons are broadly distributed throughout the entire frontal cortex, as throughout all cortex in general. Distribution of dopaminergic neurons throughout the primate frontal cortex has recently been investigated by Williams and Goldman-Rakic (1993). Among their major findings were: (1) distribution of dopamine does not respect cytoarchitectural boundaries, (2) dopamine concentration is greater in the medial regions and declines as one moves laterally, and (3) dopamine concentration declines as one moves rostrally and caudally from areas 8 and 6 (SMA). Inferior Frontal Gyrus/Cortex - (IFG)

The "anterior speech area" and "Broca's area" are two terms that are used synonymously. This dates from Paul Broca's discovery that damage to the posterior part of the IFG of the left hemisphere can cause language production problems (Broca, 1861; 1865). Unfortunately, Broca's area itself is not particularly well delimited (Broca's own patient had extensive subcortical damage), and its connection with the rather amorphously defined "Broca's aphasia" serves to muddy the waters even further (see Mohr (1976) for an in depth review of how Broca's area relates to Broca's aphasia). As such, I will define the anterior speech area as being comprised of the pars opercularis and 
the caudal part of the pars triangularis. In cytoarchitectural terms, these areas correspond to Brodmann's (1909) areas 44 and 45, respectively.

The proposed involvement of the inferior frontal gyrus is quite varied, ranging from the processing of segmented phonology, occurring in the more caudal areas (Zatorre et al., 1996; Paulesu et al., 1996) to the storage and processing of semantic information, in the more rostrally situated areas (Petersen et al., 1988; 1989). An attempt to unify the anterior and the posterior speech areas has lead to the assertion that highly structured semantic information is stored in the posterior area, whereas the mechanism needed to access and manipulate that information is located in the anterior area (Binder et al., 1993). Damage to the anterior area would result in semantic access errors while leaving the information itself intact, whereas damage to the posterior area would result in the actual loss of semantic information (Shallice, 1989). Along the same lines, Binder et al. (1994b) propose an integrative approach, suggesting that both the anterior speech area and the posterior polymodal areas comprise a distributed network, with each node in the network providing a complementary function.

Clinicians have typically associated damage to the anterior speech area with errors in speech production, a classical symptom of Broca's aphasia, whereas damage to the posterior speech area has been associated with errors of comprehension, a symptom of Wernicke's aphasia. Although this dichotomy has clinical utility, current electrophysiological work has, however, found that the anterior speech area may be involved in both speech production and comprehension. Schaeffler et al. (1996), implanted patients with subdural electrodes and found that although stimulation of the cortex of the posterior portion of the inferior frontal gyrus (reported as Broca's area) did indeed result in expressive errors, receptive errors were produced equally by stimulation of either Broca's or Wernicke's area. Similarly, other researchers have noted that stimulation of either the anterior or the posterior speech areas, can result in similar errors, such as errors in counting, inability to name objects, or the generation of phonemic or semantic paraphasias during object naming (Penfield \& Roberts, 1959; Ojemann et al., 1989). These results suggest that the anterior/posterior dichotomy might be not be as 
strong as traditionally assumed. Also suggested by these results, is the existence of a rather strong link between the anterior and the posterior speech areas.

Area 44. According to some researchers, the potential involvement of a particular area in language processing may be inferred from two different types of physical asymmetry: (1) left-right asymmetry in humans, with the area on the left assumed to be larger and more involved in language than its homologous area on the right, and (2) interspecies asymmetry, that is to say, if a given area is proportionally larger in one species than in another, when measured against surrounding areas, then this asymmetry may reflect the fact that different species may make differential demands on that particular substrate. Both of these methods of determining involvement in language processing assume a positive correlation between size of area and processing capacity, and both of these techniques have been used to explore BA 44. As an example of the first type of asymmetry study, Galaburda (1980) found BA 44 in humans to be larger on the left than its homologue on the right. Another left-right asymmetry study by Scheibel (1984) compared the degree of dendritic branching found in layer III pyramidal neurons of the pars opercularis and caudal pars triangularis in eight recently deceased subjects. Scheibel hypothesized that a within-subjects comparison of the left opercular region with the right homologous region, should show a greater degree of dendritic complexity on the left, due to this area's known involvement in speech, and in light of the asymmetry found by Galaburda (1980). Tissue samples, although taken from both areas 44 and 45 , were primarily representative of area 44 (pars opercularis). The findings showed that, although the total dendritic length between the two areas did not differ significantly, the left side did show that its total dendritic length was comprised of a greater number of higher order branches. The author interpreted the greater degree of branching found in left opercular level III pyramidal neurons to reflect a potential for more complex information processing.

Investigation of the cytoarchitecture of BA 44 in humans by Petrides and Pandya (1994) found this area to have a very poorly developed layer IV (the granular layer), thus indicating a paucity of thalamic inputs, as well as very large pyramidal cells located in the lower part of layer III, and a densely packed layer II. These investigators also were able 
to isolate a homologous area in the monkey, i.e. an area with similar cytoarchitecture, located in a similar position in the frontal lobe. The monkey homologue was determined to be situated on the posterior bank of the lower ramus of the arcuate sulcus. This area in the macaque is much smaller than BA 44 in the human, potentially pointing to its unique contribution to language processing. Connections between this area and other areas in the macaque monkey were investigated by Deacon (1992). Deacon found this area to connect mainly with motor areas such as the premotor, supplementary motor and the precentral motor cortex, although some parietal connections to the inferior parietal cortex and the dorsal opercular bank of the Sylvian fissure were also noted. Frontal connections were sparse and consisted of areas to which area 45 also projected, such as the sulcus principalis, the ventral lateral prefrontal cortex, the anterior cingulate, and the dorsal insular cortex.

Insert Figure 2 about here

Various researchers conducting activation studies have implicated left BA 44 and BA 45 in phonetic segmentation (Zatorre et al., 1996; Paulesu et al., 1996), a subvocal rehearsal system (Paulesu et al., 1993), a store for articulatory representations or to provide access to such a store (Zatorre et al., 1992), and an intentional system (Friston et al., 1993). An examination of BA 44 activations suggests that they might be divided to two qualitatively different types; those located quite close to the premotor cortex, and thus strongly associated with articulatory programming, and those more rostrally situated activations that appear to be more involved in phonetic segmentation (see Figure 2). An example of the former is provided by Paus et al. (in press), who, during a phoneme production task, did not find BA 44 activation, but did report a left precentral gyrus activation. Evidence that rostral BA 44, conversely, is less involved in articulatory programming, is given by Paulesu et al. (1996), in finding left BA 44 activation during a rhyming and a short-term memory task that required no verbal response. Of the remaining activations, most of them are the result of auditory stimulus presentation, and 


\section{Comparing Models}

26

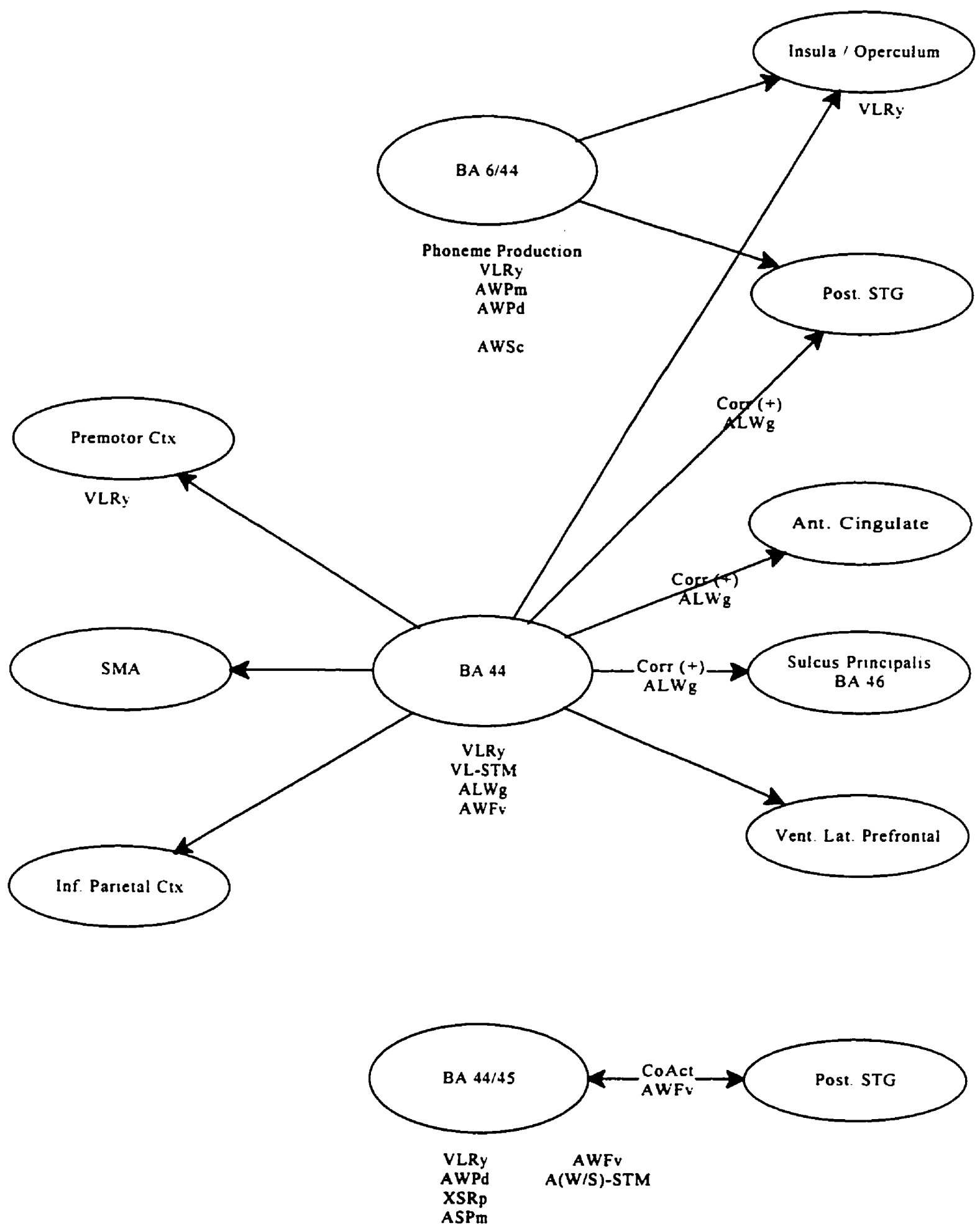

Figure 2 
are located either in mid BA 44, or near the boundary of BA 44 and BA 6 , with the phonological tasks generally situated caudally, and the semantic tasks situated rostrally. Phonological activations were noted during phonemic discrimination tasks (Zatorre et al., 1992), rhyming tasks (Paulesu et al., 1993; 1996), and phonemic monitoring tasks (Zatorre et al., 1996), whereas more rostral semantic activations were noted for tasks involving the generation of a word in response to a letter stimulus (Friston et al., 1993), and a verb fluency task (Warburton et al., 1996).

Activations occurring at the boundary of BA 44 and BA 45 are similar in nature to those found in BA 44, i.e. most of the activations resulted from auditorily presented stimuli, and most of the tasks had a strong phonological component. The phonological tasks included tasks such as rhyming (Paulesu et al., 1996), phonemic discrimination (Zatorre et al., 1996), phonemic monitoring (Demonet et al., 1992), and the silent repetition of pseudowords (Warburton et al., 1996). The only tasks noted to produce activations in this area that had a strong semantic component were verbal fluency tasks (Wise et al., 1991, Warburton et al., 1996), and a task in which subjects were required to make concrete/abstract judgments on visually presented nouns (Desmond et al., 1995). Tasks requiring semantic categorization (Wise et al., 1991), or category fluency (Warburton et al., 1996) did not result in BA $44 / 45$ activations, thus questioning the role that this area plays in making subordinate category judgments.

Area 45. A number of studies examining left-right BA 45 asymmetries in humans, as well as inter-species BA 45 asymmetries have been done. In a post mortem study of cytoarchitectonic asymmetries of BA 45 in 19 human brains, Hayes and Lewis (1995) found that the pyramidal cells of layer III had a significantly larger cross-sectional area in the left hemisphere than in the right. The authors interpreted this asymmetry to indicate that the larger layer III pyramidal cells might potentially be involved in certain lateralized aspects of motor speech function. Application of the concept of root, core, and belt lines (Galaburda \& Pandya, 1983), in which information is fed forward from layer III into layer IV, would argue for the possibility of information flow from area 44 layer III rostrally into layer IV of area 45. Conversely, information could flow from area 45 to area 44 via feedback linkages from layer VI to layer I. In another investigation of the degree of 
asymmetry of the pars triangularis, Foundas et al. (1996) found that, of 11 patients, who had previously undergone sodium Amytal testing in order to determine language lateralization (10 with left hemisphere language, 1 with right hemisphere language), volumetric analysis of their MRI scans identified 9 of the 10 left hemisphere language patients to have had a significant leftward pars triangularis asymmetry, and showed the 1 right hemisphere patient to have had a significant rightward asymmetry. The researchers also note that for 10 of the 11 patients, the direction of pars triangularis asymmetry coincided to the direction of planum temporale asymmetry, thus linking both pars triangularis and planum temporale asymmetry to speech production, as measured by sodium Amytal testing.

The architectonics of BA 45 in both humans and the monkey was explored by Petrides and Pandya (1994). Cytoarchitectonically, this area differs from BA 44 in that it has a very well developed layer IV. Lower layer III pyramidal cells are well represented and layer II is also well developed, just as in BA 44. Minor cytoarchitectonic differences within BA 45 resulted in the authors subdividing this area into a rostral part (45A) and a caudal part (45B). A cytoarchitectonically similar area was noted in the monkey, and was thus similarly subdivided into area $45 \mathrm{~A}$, lying on the anterior convexity of the arcuate sulcus, and area 45B, lying within the rostral bank of the arcuate sulcus. Of interest is that the caudal area $45 \mathrm{~B}$ is relatively much larger in the human than in the macaque, a finding which is similar to that for BA 44, whereas the more rostral area $45 \mathrm{~A}$ does not seem to show this inter-species asymmetry to the same extent. This finding is in accordance with the belief that the area critical to language processing in the left frontal lobe is comprised of the pars opercularis and caudal pars triangularis (areas 44 and 45B). In accordance with the above findings, Deacon (1992) found area 45B in the monkey to connect primarily with prefrontal and auditory cortex, as opposed to the primarily motor related connections reflective of area 44 . Of particular interest is that connections were noted between this area and the superior temporal gyrus as well as the dorsal bank of the superior temporal sulcus, as depicted in Figure 3. This implies that the granular layer of area 45B might be the primary inferior frontal receiving area for information transported from the STG via the arcuate fasciculus, in accordance with the Geschwind model. 


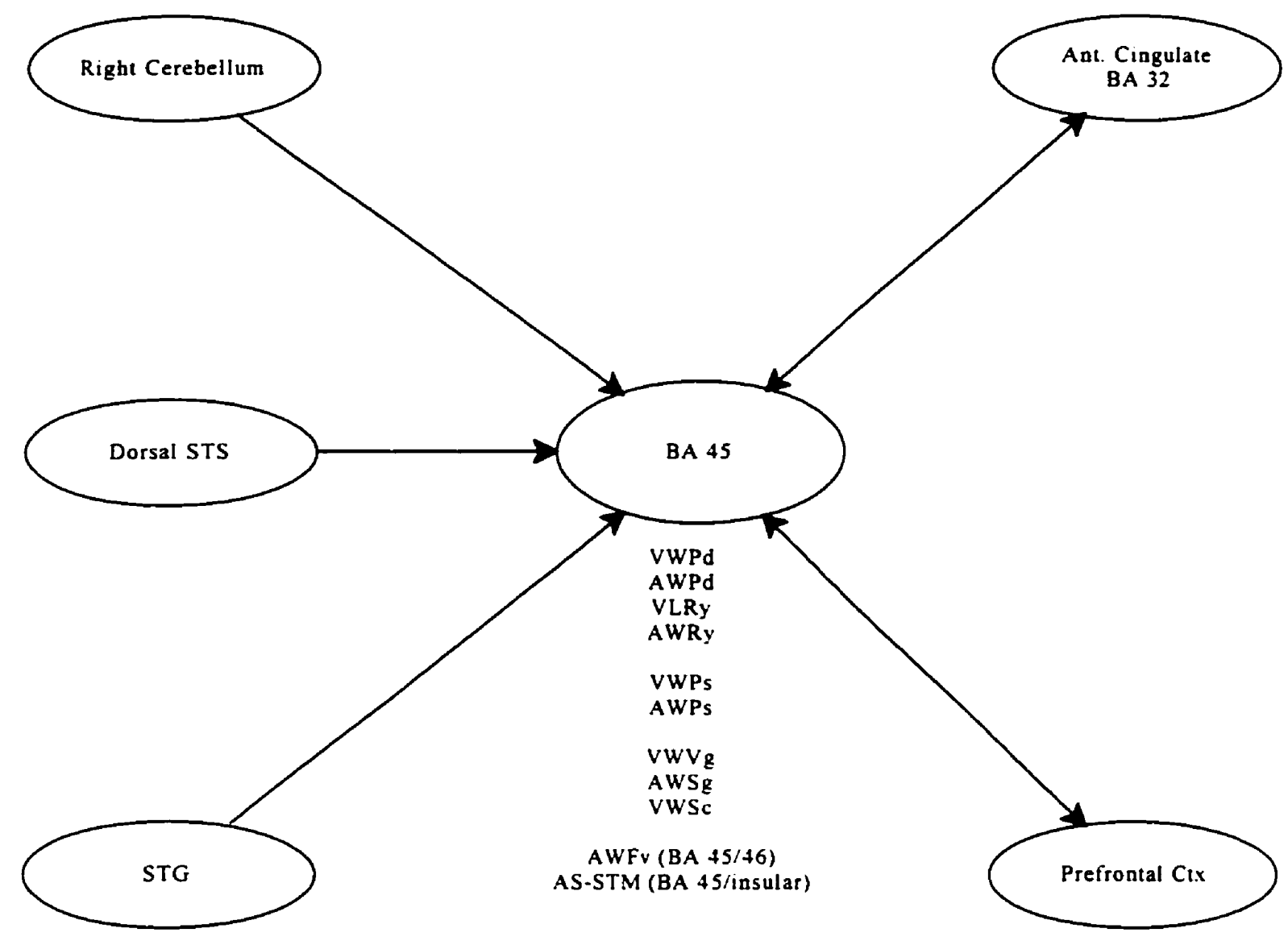

Figure 3 
The functional contribution of BA 45 is difficult to define because most researchers do not attempt to differentiate BA 44 contributions from those of BA 45. As such, many of the functions that have been attributed to BA 44, are also attributed to BA 45 . Nonetheless, some similarities as well as differences between these two areas can be noted. The primary similarity between these two areas is in that phonological tasks such as phonemic discrimination (Fiez et al., 1993; Zatorre et al., 1996) and rhyming (Klein et al., 1995, Paulesu et al., 1996) can result in either BA 44 or BA 45 activations. BA 45 activations differentiate themselves from those of BA 44 in that they appear to be more involved in both active and passive tasks in which words, rather than letter strings or pseudowords, are processed, thus indicating semantic-related processing. As was the case for the more active tasks, passive activations were not dependent upon input modality (Petersen et al., 1990; Zatorre et al., 1992). The active tasks consisted of verb generation (Petersen et al., 1989; Raichle et al., 1994), synonym generation (Klein et al., 1995), and semantic categorization (Kapur et al., 1994). A lone verb fluency activation was found near the boundary with BA 46 (Warburton et al., 1996), in accordance with the accepted role of the dorsolateral prefrontal cortex in fluency tasks. As such, BA 45 appears to be more involved in semantic processing than fluency.

Areas 47 and 11. The possible involvement of BA 47 in language related processing is indicated by the fact that it's located very close to the areas classically associated with language production, i.e. just inferior to BA 45 , as well as inferior and rostral to BA 44 . It should be noted, however, that BA 47 activation is not found nearly as frequently as BA 45 or BA 44 activations, perhaps as a result of the nature of the activation tasks used.

Petrides and Pandya (1994) defined an area, area 47/12, that they identified as being cytoarchitectonically homologous in both man and macaque. This area does not appear to show any significant relative inter-species asymmetry, thus allowing us to propose that this area might serve the same functional purpose in both human and non-human 
primates. Cytoarchitectonically, area $47 / 12$ is defined by a distinct layer II and a well developed layer IV. Layer III contains small and medium sized neurons in the upper part and larger pyramidal neurons in the lower part. Layers V and VI contain small and medium sized pyramidal neurons.

BA $47 / 11$ activations can be characterized by the scarcity of activations related to phonological processing, a lack of activations resulting from verbal fluency tasks, a tendency for the tasks to be visual rather than auditory, particularly with regard to BA 11 activations, and a predominance of semantic tasks. Semantic tasks include semantic categorization (Kapur et al., 1994), verb generation (Petersen et al., 1989), semantic monitoring (Petersen et al., 1989), and synonym generation (Klein et al., 1995). Passive tasks involving the presentation of words have also resulted in BA 47 activation (Petersen et al., 1990; Zatorre et al., 1992). Those who believe that the role of left BA 47 is primarily semantic in nature, propose that these activations, in response to passive word presentation, reflect the automatic activation of the semantic system (Posner \& Snyder, 1975; Petersen et al., 1990; Zatorre et al., 1992; Price et al., 1996). Also of interest, and contrary to other language-related prefrontal areas, right-sided BA 47 activations have also been noted, although most of these activations have been the result of tasks with a prominent memory component, such as retrieval from episodic, but not semantic, memory (Fletcher et al., 1995), or maintenance in short-term memory of both words or pseudowords (Fiez et al., 1996b), thus indicating functional lateralization of left and right BA 47.

\section{The Parietal Lobe}

The parietal lobe of the left hemisphere is historically associated with language processing for a number of reasons. Firstly, the rather lose definition of Wernicke's area, an area whose damage is frequently associated with fluent aphasia, has allowed this area to encompass not only the posterior third of the superior temporal gyrus as defined by Geschwind (1969), but also the angular gyrus and the supramarginal gyrus, both of which are located within the inferior parietal lobe. Secondly, the angular gyrus, a parietal area which corresponds with area 39 of Brodmann (1909), plays a critical role in the disconnection theory model of language as proposed by Geschwind (1965). 


\section{Inferior Parietal Lobule (IPL) : Supramarginal Gyrus}

Isolating the function of the supramarginal gyrus (roughly equivalent to BA 40) on the basis of human lesion data is rather difficult due to its proximity to the posterior STG and the angular gyrus, and that focal lesions of the supramarginal gyrus are rare. Caplan et al. (1995), in an attempt to correlate symptomology with posterior speech area damage, performed a morphometric analysis of the MRIs of 10 aphasic patients, who had presented with difficulties in phonemic discrimination. The researchers found that damage to the left supramarginal gyrus and/or the bordering parietal operculum was most often associated with errors in phonemic discrimination and identification.

Activation studies have supported the proposal that the left supramarginal gyrus is primarily activated during the execution of tasks with a phonological component, such as orthographic fluency (Rueckert et al., 1994), phonological short-term memory access (Paulesu et al., 1993; 1996), complex phonemic discrimination or phonemic monitoring (Demonet et al., 1993; Zatorre et al., 1996) or feature detection during display of a letter string (Price et al., 1996). Activations occurred without regard to presentation modality, and all were lateralized to the left. These findings support the claim of Paulesu et al. (1993; 1996), that left BA 40 functions primarily as a short-term phonological store. Arguing against the role of the supramarginal gyrus in general phonological processing, Paulesu et al. (1993) report left BA 40 activation during a short-term memory task that required access to the phonological store, but not during a letter rhyming judgment task, that did not require such access. The authors conclude that left BA 40 is the site of the short-term phonological store component of the articulatory loop system, and that it will always activate when faced with a task that has a phonological short-term memory component.

\section{Inferior Parietal Lobule (IPL): Angular Gyrus}

It should be noted that although the angular gyrus has been implicated in language processing, from a clinical point of view, damage to the left angular gyrus is primarily associated with Gerstmann's syndrome (Gold, 1995). Gerstmann's syndrome is associated with left-right confusion, alexia, agraphia, and acalculia. The aforementioned symptoms serve to point to the involvement of the left angular gyrus in language, as well 
as its apparent involvement in body-centered spatial processing. Dejerine (1891) suggested that the left angular gyrus was the locus of visual word images, i.e. a visual word lexicon. Damage to the left angular gyrus results in both alexia and agraphia (Benson \& Geschwind, 1969). Geschwind (1965) believed that the left angular gyrus contained rules that were used to either recode visual word forms into their phonological equivalents or vice versa. He also believed that the visual word form to phonology recoding was essential in order to access either the anterior speech area or the semantic system.

The areas of greatest interest in the IPL, with regard to language processing, is the multimodal area located in the caudalmost part of the IPL, consisting of the caudal part of area PG and area Opt. This area can be considered as containing, in part, the angular gyrus and Brodmann's area 39. Efferent fibers from this area have been found to project to area 46 and to the dorsal areas 8 and 6 in the frontal lobes. (Pandya \& Yeterian, 1990), the association cortex of the upper bank of the STS (areas TPO and PGa), area Tpt (Pandya \& Sanides, 1973), the caudal cingulate gyrus (area 23), and the parahippocampal gyrus (areas TF, TH, and TL) ( Pandya et al., 1988). Afferents are received from the posterior cingulate (area 23) (Pandya et al., 1988) and from the inferior temporo-occipital region (Henderson et al, 1986).

Prior to presentation of the activation studies demonstrating activation in the area of the angular gyrus, one should first take note of a relevant finding made by Muri et al. (1996). In a recent AMRI study, Muri et al. (1996) claim to have identified the locus of the human posterior eye field (PEF), reportedly located deep in the intraparietal sulcus, bordering Brodmann areas 39 and 40 . Of note is that this area is very close to the area which has been shown to activate in response to reading visually presented words. As such, and assuming that there is no lateralization of function within the PEFs, bilateral activation in this area, or activation of this area in concert with a frontal eye field, must always be regarded as potentially reflecting the activity of the PEFs, rather than the activation of a "visual word store". To illustrate this point, Demonet et al. (1994) found bilateral activation of the angular gyrus during execution of a lexico-semantic task. This result suggests either (1) that the bilateral activation reflects activation of the posterior 
eye fields (Muri et al., 1996), and/or (2) that a connection may exist between the left and the right angular gyri, and that this connection is not inhibited during lexico-semantic processing. Other studies have similarly found either bilateral angular gyrus activation (Kapur et al., 1994; Demonet et al., 1993), or angular gyrus activation in concert with left frontal eye field (BA 8) activation (Demonet et al., 1992; 1993). Bilateral angular gyrus activation during semantic tasks has led these authors to argue for the involvement of left and right angular gyri in semantic processing, even though bilateral angular gyrus activation has also been noted during the performance of a phonemic discrimination task (Kapur et al., 1994). Alternatively, it could be argued that these particular angular gyrus activations might be more associated with eye movement and the inclination of subjects to move their eyes during demanding tasks.

Insert Figure 4 about here

Researchers performing activation studies have proposed that the angular gyrus may be (1) an area important for semantic processing (Demonet et al., 1992; 1993), (2) a "word form area" (Menard et al., 1996), in support of Dejerine (1891), and (3) an area involved in the phonological coding of both auditorily and visually presented words (Petersen et al., 1990), in an extension to the proposal of Geschwind (1965). As can be seen in Figure 4, most of the tasks associated with angular gyrus activation are semantic in nature, although activations have also been reported during tasks requiring passive presentation of words and pseudowords (Fiez et al., 1996a; Petersen et al., 1990), reading (Menard et al., 1996), as well as rhyming tasks (Petersen et al., 1990). Of the semantic activations, those related to fluency, such as orthographic fluency (Frith et al., 1991a), category fluency (Frith et al., 199 1a; Warburton et al., 1996), and verb fluency (Warburton et al., 1996) appear predominant. Activation has not yet been found in response to simple word repetition tasks. 
Comparing Models

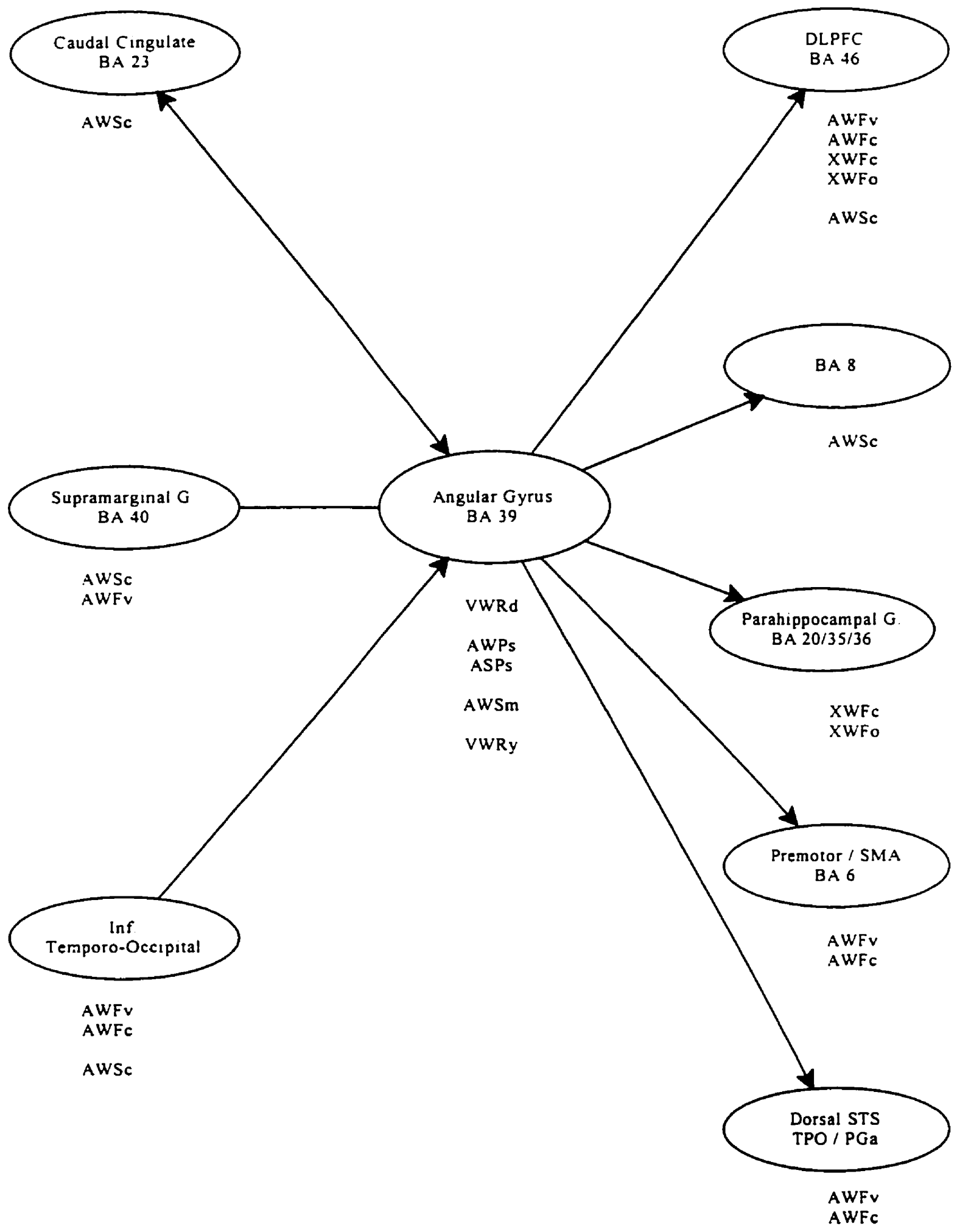

Figure 4 


\section{The Temporal Lobe}

The functional significance of the dominant temporal lobe to language processing has long been known, primarily as a result of post mortem studies of aphasic patients. The first to make the connection between language impairment, primarily in the realm of language comprehension, and damage to the posterior region of the superior temporal gyrus was Wernicke (1874). Since then, the actual area encompassed by Wernicke's area has been the source of some confusion (Bogen \& Bogen, 1976), mostly due to the fact that classical aphasiology has always been more interested in correlating rather abstract, and constantly changing, symptom profiles with neuroanatomy, rather than simply correlating specific symptoms with neuroanatomy. As a result of this approach, Wernicke's area can be interpreted as encompassing most of the middle and posterior left temporal lobe, as well as the entire inferior parietal lobe. Since this definition of Wernicke's area is obviously too broad to serve any useful purpose, the following paragraphs will refer to the various subareas that comprise the posterior speech area by either gyral anatomy or cytoarchitectonic designation.

\section{Superior Temporal Region (STR)}

The two structures of interest located on the dorsal surface of the temporal lobe consist of Heschl's gyri, the locus of the primary auditory cortex (PAC), and the planum temporale. We shall first explore the general structure of this region, prior to examining these areas in particular.

The cytoarchitecture of the STR was elucidated by Galaburda and Pandya (1983). They proposed a system of three rostrocaudal lines, starting at the temporal pole and running caudally until the end of the Sylvian fissure. These lines were designated as the root, core, and belt lines, and the detailed interconnections between these regions were expanded upon by Cipolloni and Pandya (1989). In addition to detailing both the intraand interhemispheric connections of these areas (primarily via layer III), these authors also noted that the root line received projections from the insula, and that the core and belt lines received projections from the superior temporal sulcus (STS). Since Galaburda and Pandya (1983) had previously noted the existence of projections emanating from the primary auditory areas to parasensory areas, and then on to association cortex in the STS 
and the insula, the findings of Cipolloni and Pandya (1989) suggest that the connections between PAC and multimodal association areas are reciprocal. This is of particular interest to the area of language processing, since the insula and the cortex of the STS are known to be multimodal association areas and may thus be involved in amodal language processing. Connectivity of the STR to subcortical structures is defined by (1) the existence of the expected linkage from the ventral medial geniculate nucleus (MGN) of the thalamus to the PAC, (2) the connection from the dorsal MGN to BA 42 and BA 22, as well as (3) a reciprocal corticothalamic projections from all STR areas back to the MGN (Pandya et al., 1994). STR corticothalamic projections arise primarily from layer $\mathrm{VI}$ and likely use glutamate as a neurotransmitter.

\section{Primary Auditory Cortex (PAC)}

Heschl's gyrus afferents are all subcortical, while efferents project to the insula, the frontal operculum, the planum temporale, the supramarginal gyrus, and the angular gyrus (Galaburda \& Sanides, 1980; Carpenter \& Sutin, 1983). Studies of the PAC of the cat by Prieto et al. (1994) have helped to shed some light on the cytoarchitectonic and neurochemical structure of this primary receiving area. The PAC was found to contain a high concentration of inhibitory GABAergic neurons (24.6\%), measured across all layers, with layer I showing the highest concentration at $94 \%$. Results of activation studies shall not be discussed in depth at this point, other than to note (1) that PAC activation is routinely found during auditory stimulus presentation, and (2) researchers are starting to correlate PAC activation with other regions in order to determine the extent of modality specific processing found at the site, i.e. a high correlation with PAC implies that the other area has a greater involvement in lower-level acoustic processing.

\section{Planum Temporale}

The interest in the left planum temporale (PT) in language processing stems from the finding by Geschwind and Levitsky (1968) that the planum temporale was larger on the left than on the right in approximately $65 \%$ of the cases that they examined. This finding, when combined with the finding that approximately $96 \%$ of all right-handers are lefthemisphere for language (Rasmussen \& Milner, 1975; 1977), has served to point to a possible role of the planum temporale in language processing. Although the evidence 
supporting planum temporale asymmetry is quite strong, its existence and/or its interpretation is by no means undisputed. Musiek and Reeves (1990), for example, found the planum temporale to be larger on the left, but also found a similar asymmetry for Heschl's gyrus, thus pointing to the predominance of the left hemisphere in general auditory processing, rather than language related processing. The Heschl's gyrus asymmetry found by Musiek and Reeves has recently been further explored by Penhune et al. (1996), who, in an in-vivo MRI study of PAC volume, noted that the $L>R$ asymmetry is due to an increase in left PAC white matter, reflecting greater left-sided connectivity. The volume of PAC gray matter was not found to differ significantly between hemispheres. The authors suggest that the increase in left PAC connectivity might be related to the left hemisphere's dominance for speech, thus arguing that specialized speech-level processing begins much earlier than has previously been suggested.

On a final note with regard to PT asymmetry, it has recently come to light that the technique used to measure PT volume or surface area can, itself, have an effect on whether or not an asymmetry is found. Westbury et al. (1996), for example, investigated this possibility by measuring PT asymmetry, using in-vivo MRI scans, via both a classical knife-cut method and an objective rule-based method. The results were in the expected direction, with the classical method showing an asymmetry, and the rule-based method indicating no asymmetry. This study demonstrates that studies of PT asymmetry must be interpreted with care, and close attention paid to how the area of the PT is defined and measured.

Results from activation studies are varied, and do not definitively answer the question of whether the PT does contribute to phoneme-level language processing, or whether its function is confined to general acoustic analysis, like the PAC. Evidence for some involvement of the left PT in linguistic processing was found by Paulesu et al. (1996) who found activation during a letter rhyming judgment task. Of significance was that the PT activated even though stimuli were presented visually and the subjects had to respond manually. The manual response is significant because Paus et al. (in press) also found left PT activation during a simple phoneme production task in which subjects were not allowed to hear their own speech. This activation, in the absence to speech input, 
could be explained as a result of corollary discharge emanating from frontal motor or premctor areas, although this would not explain the findings of Paulesu et al. (1996). The Paus et al. group also found that both the rate of phoneme production and bilateral putamen activation correlated positively with PT activation. These results, unlike those of Paulesu et al. (1996), emphasize the involvement of the PT in quite low-level processing, perhaps functioning to modulate the perception of one's own voice.

\section{Superior Temporal Gyrus (STG)}

Wernicke (1874) found that damage to the posterior STG as well as to cortex in the adjacent inferior parietal lobe and the posterior middle temporal gyrus (MTG) was associated with difficulties in language comprehension. Geschwind (1969), in an attempt to clarify the extent of Wernicke's area, reduced the core of Wernicke's area to include only the posterior third of the left STG, although many researchers still use a somewhat more liberal definition of the area. The posterior STG has been associated with processing at both the semantic and phonological level. Paulesu et al. (1996), for example, propose that this area is the store for whole word phonology, which is conveyed to the anterior speech area via the insula, which converts from whole worc. onology to segmented phonology en route. The notion that this area is the locus of a whole word phonology store is not dissimilar from that proposed by Geschwind (1965), although the proposed routes by which information is transferred to the anterior speech area are quite different. While not denying that the posterior STG plays a role in phonological processing, Binder et al. (1993) have proposed that this area might also be involved in lexico-semantics. They propose a dichotomy in which highly structured forms of semantic information are stored in the posterior speech area, and that access to this semantic store can only gained by the engagement of specialized retrieval mechanisms, located at the anterior speech area. The distinction between storage and retrieval is in accordance with the ideas of Shallice (1989), and receive support from lesion studies correlating site of lesion with the occurrence of a "pure" semantic deficit. In such a study, in which Hart and Gordon (1990) compared lesion sites of three patients with a "pure" semantic deficit with those of other aphasics, the authors were able to isolate an area encompassing the posterior portion of the left STG and MTG as well as the inferior 
portion of the left inferior parietal lobe, which, when damaged, always resulted in a semantic impairment. Patients with lesions only slightly encroaching into this area suffered less severe semantic deficits, implying that the lesion is associated with partial destruction of the semantic store, rather than impairment of a semantic access mechanism.

The area of the STG, which corresponds roughly to BA 22, can be defined cytoarchitectonically (Seltzer \& Pandya, 1978; Galaburda \& Pandya, 1983), or alternatively, it may be segmented on the basis on the long cortico-cortical efferents which project from it (Chavis \& Pandya, 1976). Although most of the work tracing the connectivity of this region has been done on monkeys, it is assumed that much of the connectivity between human and non-human primates is similar, if not identical. Studies, similar in nature to the work of Petrides and Pandya (1994), giving a correspondence between monkey and human cytoarchitectonics in the area of the STG, are underway and will prove helpful in future studies of human STG connectivity.

In an early attempt to map the STG, Chavis and Pandya (1976), divided the STG into three association areas, based upon cortico-cortical projections. These areas were named $\mathrm{AA} 1, \mathrm{AA} 2$ and $\mathrm{AA} 3$, proceeding in a caudal to rostral direction, and projected to $\mathrm{BA} 8$, dorsal prefrontal areas, and medial prefrontal and orbitofrontal areas, respectively. The distinct temporofrontal fiber tracts projecting from the belt regions of the STG were further highlighted by Petrides and Pandya (1988). They found that rostral areas (Pro, Tsl, Ts2) projected to medial and orbital frontal cortex via the uncinate fasciculus, whereas the fibers originating from the middle part of the STG (Ts3, paAlt) projected to lateral frontal and dorsal medial areas via the extreme capsule situated dorsal to the fibers of the uncinate fasciculus, and the fibers originating from the temporoparietal area (Tpt) projected to the frontal lobe via the extreme capsule, the arcuate fasciculus, and the superior longitudinal fasciculus (see Figure 5). Pandya et al. (1988) also found temporofrontal projections to be distributed in a similar caudal to rostral pattern as was observed by Chavis and Pandya (1976), and similarly noted that most STG belt areas also projected to polymodal and paralimbic areas as well. 


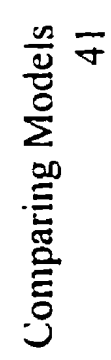

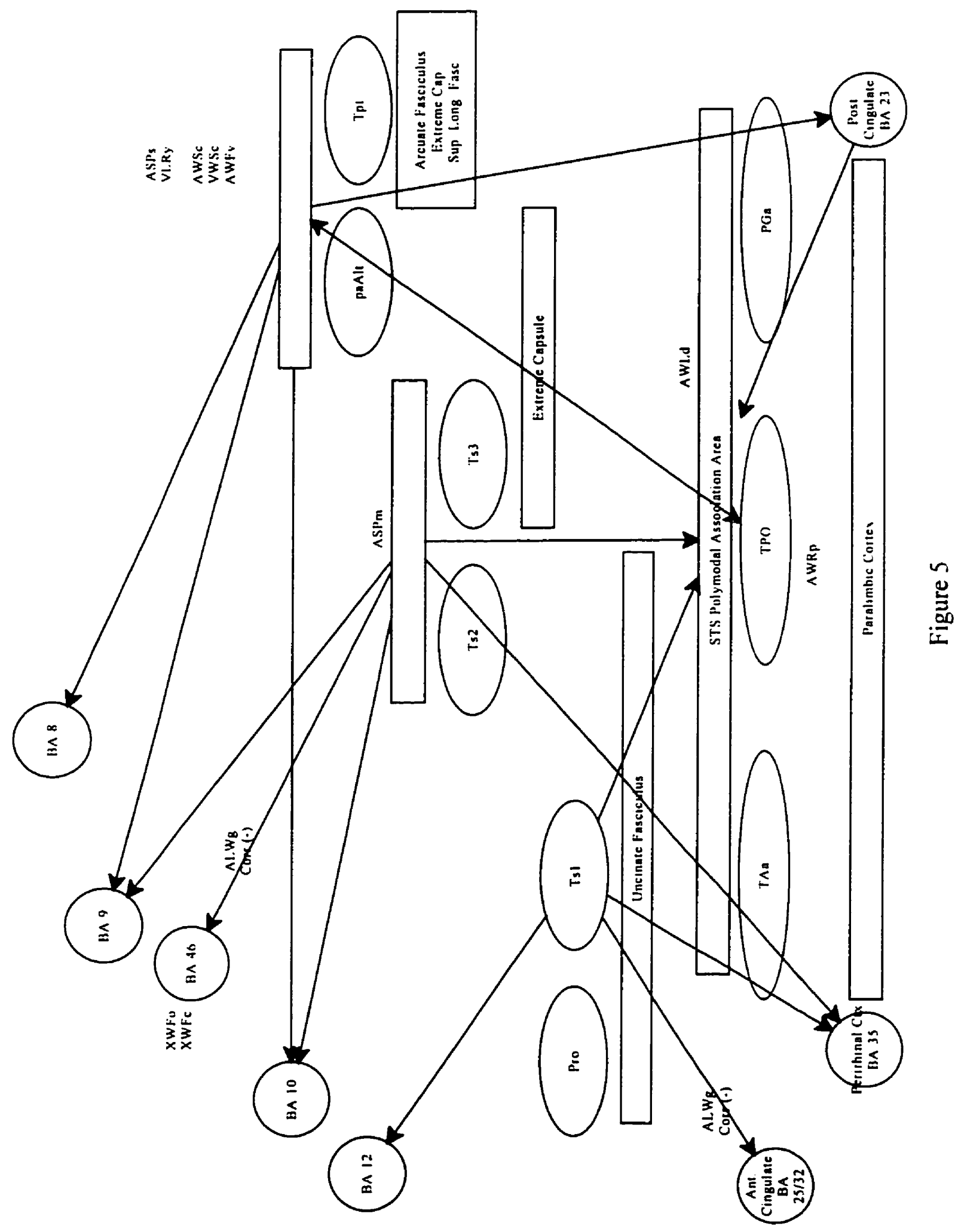


Although activation profiles for the STG are quite heterogeneous, most researchers propose a crucial role for this region in language processing. Wise et al. (1991) quite elegantly provided evidence for this by noting that left posterior STG blood flow did not correlate highly with the rate of word presentation $(r=.36)$. Conversely, other areas such as the right posterior STG and the PAC were found to correlate significantly with presentation rate. This finding was interpreted as support for the proposal that the left posterior STG is primarily involved in processing that is not strictly defined by low level stimulus related processing. This finding has been challenged by Binder et al. (19??), who found left STG to correlate strongly with presentation rate. This study, however, suffered from a number of weaknesses, such as (1) only 3 subjects were examined, and (2) the correlations were not localized to the posterior STG, i.e. some subjects showed strong rostral STG correlations, others showed medial or caudal STG correlations.

Bilateral decreases in posterior STG have been noted during semantic categorization tasks (Kapur et al., 1994), word generation tasks (Friston et al., 1993; Fletcher et al., 1995), and word fluency tasks (Frith et al., 199la), whereas bitemporal increases were found during an episodic memory encoding task (Fletcher et al., 1995) and a lexical decision task (Frith et al., 1991a). Bitemporal decreases, which are often associated with increases in BA 46 activation (Friston et al., 1993; Frith et al., 1991a), have been linked to an attentional shift away from modality specific processing (Kapur et al., 1994; Frith et al., 1991a; 199lb) to higher level, possibly semantic, processing. Fletcher et al. (1995), noting bitemporal increases during an episodic memory encoding task and bitemporal rCBF decreases during a retrieval task, interpreted these findings to reflect that more phonological and semantic processing occurs during initial encoding than during retrieval. Frith et al. (1991a) had a somewhat different interpretation for the bitemporal increases found during a lexical decision task. The increases, according to the authors, reflected a spreading activation through an auditory word lexicon located in the superior 
temporal cortex. Blood flow reduction in the STG together with BA 46 activation during a word generate task, thus reflected BA 46 inhibiting inappropriate lexical items.

A semantic role for the posterior STG was proposed by Wise et al. (1991) who found that the presentation of both words and pseudowords resulted in similar activations during tasks that involved passive semantic processing, such as listening to the passive presentation of pseudowords, and those that required more active semantic processing, such as semantic categorization and verb fluency. The authors reasoned that, since no frontal activations were observed during the semantic tasks, as would be predicted by Petersen et al. (1988; 1989), semantic processing must have been carried out in the STG.

Activations related to phonological processing, which seem to be more strongly lateralized to the left STG, have been observed during tasks requiring rhyming judgment (Paulesu et al., 1996), phonemic monitoring (Demonet et al., 1992), and the auditory presentation of words and pseudowords (Fiez et al., 1996a). Fiez et al. (1996a) see the role of the posterior STG region to be much more phonological in nature than semantic, postulating that it functions as a form of short-term storage for speech information. This is somewhat in accordance with the view of Paulesu et al. (1996) that the left supramarginal gyrus serves as a phonological store.

\section{Superior Temporal Sulcus (STS)}

The STS serves as a multimodal association area (Galaburda \& Pandya, 1983), and has both afferent and efferent connections with the belt and core lines of the superior temporal region (Cipolloni \& Pandya, 1989). Afferents are received on the upper bank of the STS (areas TPO and PGa) from the posterior cingulate cortex (area 23) (Pandya et al., 1988).

Activation patterns for the STS are similar to those of the STG. Bilateral activations were noted during the execution of tasks that might activate semantics, such as lexical decision (Frith et al., 1991a), repeating auditorily presented words (Howard et al., 1992), and passively listening to pseudowords, words, and text passages (Binder et al., 1994a). Both Frith et al. (1991a) and Howard et al. (1992) argue that these results provide support for the hypothesis that the auditory input word lexicon is located in the area of the STS. Although purely phonological tasks appear less likely to result in STS activation, 
Demonet et al. (1992) were able to demonstrate mid-STS activation during a phonemic monitoring task, possibly arguing against the existence of a whole word lexicon at this location.

\section{Middle Temporal Gyrus (MTG) / Temporo-Occipital Junction}

Hart and Gordon (1990), in a study of aphasics with pure semantic deficits, found that the area of overlap of the lesion sites encompassed the left posterior $25 \%$ of BA 22 and BA 21, as well as a small amount of rostral BA 39 and BA 37. The authors referred to this area as the left posterior temporo-parieto-occipital junction. All patients with lesions that encroached upon this area showed semantic deficits, whereas patients without semantic deficits never had lesions that invaded this area. The authors interpreted this finding as conclusive proof for the essential role that this area must play in semantic processing. Unfortunately, the results of activation studies do not point quite as clearly to the involvement of this region in semantic processing.

For the purpose of examining activations, this region shall be defined as being comprised of BA 21 and the lateral surface of BA 37. An inspection of the characteristics of the activation tasks, yield very little in common between them. Activations have been obtained by both auditory and visual presentation of words and pseudowords alike, although tasks in which words were presented auditorily seem to be somewhat more predominant. With regard to the activation tasks themselves, activations have been obtained for passive presentation of words (Zatorre et al., 1992), word repetition (Howard et al., 1992), phonological monitoring (Demonet et al., 1992), semantic categorization (Demonet et al., 1992), verb generation (Raichle et al., 1994), verb fluency (Warburton et al., 1996), and visual feature detection (Price et al., 1996). Most activations were lateralized to the left, however, some right-sided activations have been reported as well (Demonet, 1992; Price et al., 1996).

Howard et al. (1992) claim to have located both the auditory and the visual word lexicons in the area of the posterior MTG and STS. The first activation, which resulted from what, in effect, was simple repetition of auditorily presented words, was found on the posterior left MTG, and proposed to be the site of the auditory word lexicon. The second activation, which was subsequently claimed to be the locus of the visual word 
lexicon, resulted from performance of a simple reading task. The two activations were separated by approximately $16 \mathrm{~mm}$, with the auditory word lexicon situated $10 \mathrm{~mm}$ rostral and $12 \mathrm{~mm}$ inferior to the proposed visual word lexicon. Of some interest is the fact that Raichle et al. (1994) found an activation situated between the two reported by Howard et al. (1992) during the performance of a task in which subjects were required to generate a verb in response to the visual presentation of a noun. Although this activation verified the importance of the posterior left MTG in the performance of a language task, it was of some concern that Petersen et al. $(1988$; 1989) did not find a similar activation, although an identical activation task was used. Raichle et al. explain this difference in findings by noting that the area only activated after the presentation rate had been slowed from 1 word every sec, to 1 word every $1.5 \mathrm{sec}$, implying that this area can only be fully activated when given enough time to access the lexico-semantic network.

\section{Models of Language Processing}

Although the neuroanatomical and functional data presented previously serve to give us much information with regard to which areas are connected to other areas, and which areas seem to activate in response to stimuli or tasks with certain attributes, we still have yet to present an integrated view of how language is processed. That is to say, we need to create a construct that will allow us to make predictions regarding how the system, i.e. the interconnected network of language-related substrates, will function, given a specific stimulus, or alternatively, how the system will function following damage to any of the postulated components. A number of models which allow us to make predictions of this nature already exist, and are primarily of two types: those that have evolved over many years of patient study, and are thus supported by clinical neurology, and those much more recently developed models proposed by cognitive psychology. The following paragraphs will explore two such models that shall be the focus of the remainder of this paper, the clinically-based model of Geschwind (1965; 1969), i.e. the Geschwind model, and the cognitively-based model of Petersen et al. (1988; 1989), i.e. the Petersen et al. model. 
Serial Single-Route Model (Geschwind, 1965: 1969)

\section{Model Overview}

In a seminal article published in 1965, Norman Geschwind outlined his disconnection theory, as well as how this theory might be applied to the development of a neurologically plausible model of language processing. The hallmarks of this model, illustrated in Figure 6, are (1) discrete processing modules, which are associated with serially activated discrete substrates, i.e. a given stimulus is completely processed by one stage prior to being passed on to the next, (2) most, but not all, of the links between substrates are unidirectional, and (3) the essential contribution of the angular gyrus.

Insert Figure 6 about here

\section{Neuroanatomy}

Although this model allows for stimuli to enter the system from tactile, visual, and auditory modalities, this description will focus on the pathway critical to auditory input and oral output of speech. Since the actual flow is shown in Figure 6, I will simply highlight some of the assumptions that this model makes. Firstly, input is assumed to enter the cortex at BA 41 (the primary auditory cortex), passed on to BA 42 (the paraauditory cortex) and subsequently to BA 22 (the auditory association cortex) in a strictly serial fashion. Since BA 42 is assumed to be the primary callosal receiving and sending area, speech received at right primary auditory cortex is assumed to be transferred from right BA 42 to left BA 42 for subsequent language-related processing in the left hemisphere.

The auditory association cortex (BA 22) is defined as being equivalent to Wernicke's area, and has been localized primarily to the posterior portion of the left superior temporal gyrus. Geschwind postulates that this area serves as a storehouse of auditory associations, i.e. a modality-specific auditory lexicon, from which associations stored in other modalities can be triggered, via a pathway through the angular gyrus. It should be pointed 
Comparing Models

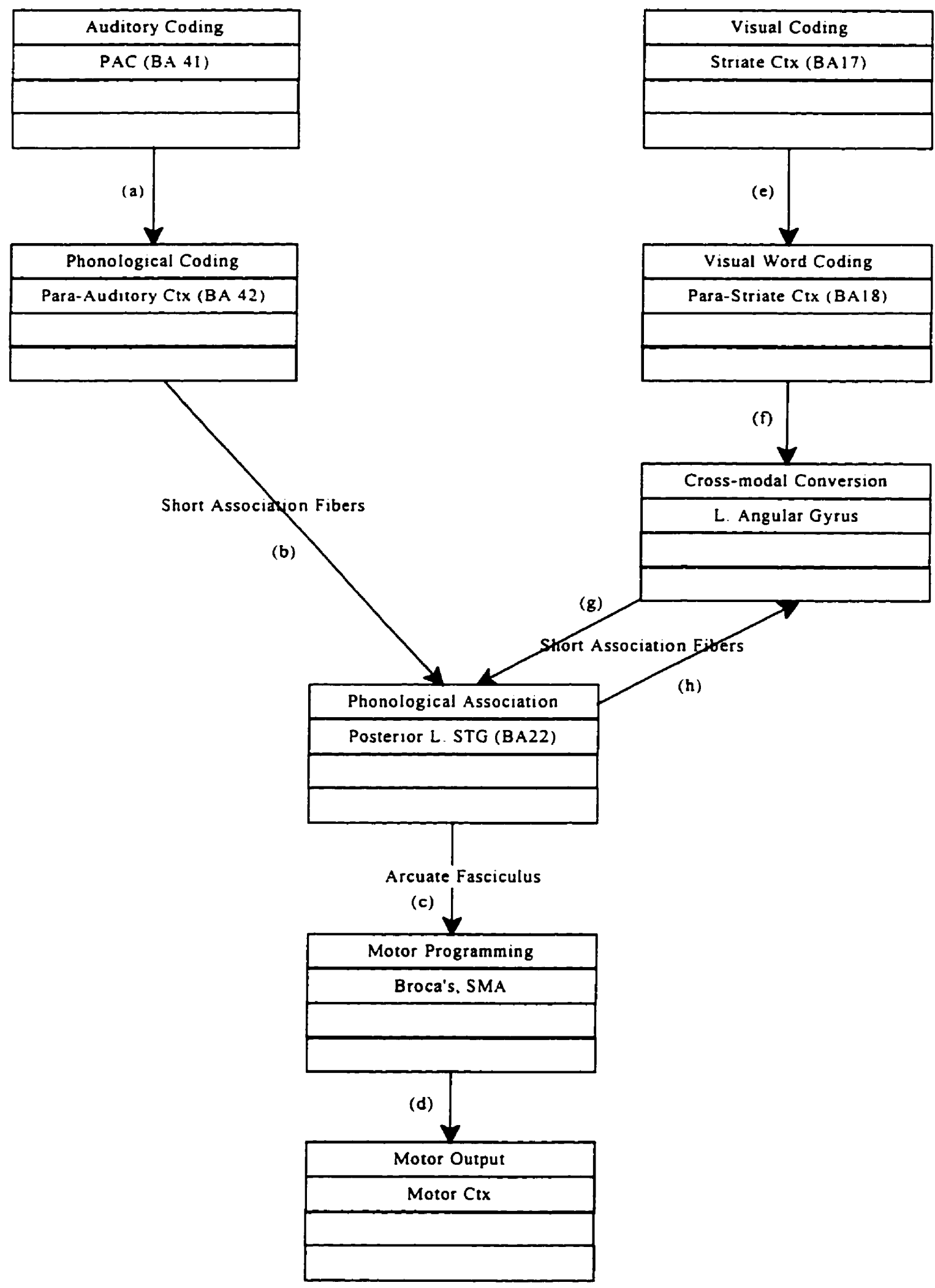

Figure 6 
out here that although many authors have interpreted the Geschwind model to implicate this area in "semantic" processing, although Geschwind himself was quite clear in declaring this area to be involved only in within-modality association. In fact, Geschwind et al. (1968) presented a case of isolation of the speech area in which, although all areas critical to language, including posterior BA 22 , were still intact, the authors state that the inability of the speech areas to stimulate multi-modal associations made comprehension impossible. This implies that the authors felt that semantics, which is obviously necessary for comprehension, cannot be localized to a specific modality, and thus cannot be wholly contained within BA 22 .

The temporo-parieto-occipital junction plays perhaps the most significant role in this model. This area, which also roughly corresponds to BA 39 and the angular gyrus, is postulated to contain rules by which information in one modality is translated into another, thus making the arousal of cross-modal associations, and therefore comprehension, possible. As such, the model allows for visual or somatosensory stimuli to invoke words stored in posterior BA 22, or conversely, words in posterior BA 22 can invoke the memory of previously stored visual or somatosensory stimuli. This bidirectional flow of information makes necessary one of the few explicitly declared reciprocal connections, i.e. those between modality-specific association areas and the angular gyrus.

The final language-related stage of processing postulated by Geschwind is localized to Broca's area, an area that contains the learned rules for translating a particular sound into a motor sequence. Speech information is transferred to Broca's area, which Geschwind defined as being located immediately adjacent to BA 6 and can thus be equated to BA 44, via the arcuate fasciculus from posterior BA 22. The arcuate fasciculus path, as presented by Geschwind, appears to be unidirectional, running from BA 22 to BA 44; the model does not anticipate any need for a reciprocal connection. Critique

The Geschwind model is still the dominant model of language processing in clinical neurology. The model is not, however, without its weaknesses, some of which were acknowledged by Geschwind (1969) himself. Geschwind, for example, noted that since 
many actions require a combination of input from many different modalities prior to motor programming, he suggested that the inferior parietal lobe might also play a role in inter-modality information mixing. He also noted that the middle temporal gyrus (MTG), not included in the original model, might be involved in naming, particularly in tasks in which the target name can only be found by a combination of a number of clues, each of which by themselves would be insufficient to complete the task. The involvement of the MTG is defended by noting that it is continuous with the angular gyrus, which does play a significant part in the model. Unfortunately, these potential modifications to the 1965 model were not specified in enough detail, i.e. no precise localization or information about hypothesized connectivity, to allow them to be implemented in a testable model.

Another possible problem with the model lies in its assumption that the inferior lateral cortex adjacent to BA 6 , which I have previously identified to be BA 44 , is the main receiving area of posterior BA 22 efferents, via the arcuate fasciculus. The possible contribution of the arcuate fasciculus is not in question, since Petrides and Pandya (1988) found, in work on the monkey, that there were indeed temporofrontal fibers which originated in area Tpt of the temporoparietal area and projected to frontal areas via this fiber tract (among others). The problem lies with the contention that BA 44 is the prime receiving area of the temporofrontal efferents. In a cytoarchitectonic study of the monkey frontal lobes, Petrides and Pandya (1994) found layer IV to be poorly developed, indicating that this area would seem to be an unlikely candidate to receive a projection of long association fibers. This is supported by the findings of Deacon (1992) who found only sparse connections between BA 44 and the temporal lobes. The findings of these researchers do, however support an alternative area, BA 45, which was found to have both a well developed granular layer (Petrides \& Pandya, 1994), and strong connections from BA 45B to many auditory areas, the dorsal bank of the superior temporal sulcus in particular (Deacon, 1992).

Another challenge to the Geschwind model has come from the functional neuroimaging work of Petersen et al. (1988, 1989). Petersen et al. scanned subjects both while repeating words and while generating an appropriate verb to a presented noun. Stimuli were presented auditorily and visually. The authors noted the following 
incongruities with the Geschwind model: (1) the model predicts that visually presented words must be converted in the angular gyrus and then arouse the equivalent phonological representation in posterior BA 22 prior to output; no such activations were found, and (2) the verb generation task activated a left frontal, rather than the predicted posterior BA 22 site. Although these criticisms of the Geschwind model have, themselves, been criticized; with critics arguing that the verb generation task may not have been an effective manipulation, or that the scanner may not have been sensitive enough, or that the subtraction that was done may have been inappropriate, it does, nonetheless, point to the potential of using activation studies to test models in neurologically intact subjects.

On a somewhat more positive note, it should be noted that the Geschwind model, though clinically-oriented, does share much in common with some cognitively-oriented models, such as that of Ellis and Young (1988). For example, according to the Geschwind model, phonological processing occurs in the para-auditory cortex adjacent to the primary auditory cortex and then moves on to the phonological association stage located in the posterior STG, regarded to be the locus of Wernicke's area, and finally on to the motor programming stage. This configuration shares much in common with the Ellis and Young model in that (1) Geschwind's phonological coding stage is analogous to Ellis and Young's auditory analysis system; a phoneme extraction stage, (2) the phonological association stage could be regarded as serving a similar function as Ellis and Young's auditory input lexicon, (3) the motor programming stage could be considered as containing Ellis and Young's speech output lexicon, and (4) a unidirectional link exists between the phonological association stage (auditory input lexicon) and the motor programming stage (speech output lexicon) via the arcuate fasciculus. Many other differences separate these two models, however, the similarities do point to the potential for a reform of the Geschwind model.

Parallel-Route Model (Petersen et al., 1988, 1989)

\section{Model Overview}

The model of Petersen et al. is unique in that it is based upon models of language processing developed by cognitive psychology, and in that an attempt was made to test 
the predictions of the model using a non-clinical population. In accordance with cognitive models, such as that proposed by Ellis and Young (1988), Petersen et al.'s model postulates (1) separate modality dependent pathways for auditory and visual language input, (2) the ability for visual and auditory processing to occur concurrently, (3) the existence of separate input and output lexicons, and (4) the direct access to output modules without the need to route information through semantics.

Insert Figure 7 about here

\section{Neuroanatomy}

Figure 7 summarizes the findings of Petersen et al., noting both the proposed functional contribution of each module, as well as associated substrates. Neural substrates are described via reference to gyral anatomy, cytoarchitectonic area, and stereotactic coordinates according to the atlas of Talairach and Tournoux (1988). As stated previously, most salient is the fact that this model postulates the existence of separate auditory and visual language processing paths. With regard to the auditory pathway, auditory input undergoes basic non-language specific processing in the primary auditory cortex; the output of which is then passed to the temporoparietal cortex for phonological coding. It should be noted that, according to this model, the temporoparietal cortex is the stage at which language-specific processing first occurs. Once the phonological code has been generated, the code may then be used to access the semantic system (BA 47) directly, or to access the output processing stages, which can be accomplished either directly or indirectly via the semantic system. A parallel input pathway has been proposed for visual word input. Processing begins at the striate cortex, the output of which is passed to the left medial extrastriate cortex for the generation of visual word related codes. The processing that occurs at this stage is analogous to the processing which occurs at the temporoparietal cortex in the auditory pathway, and therefore similarly, this stage is the first language-specific processing stage. As is the 
Comparing Models

52

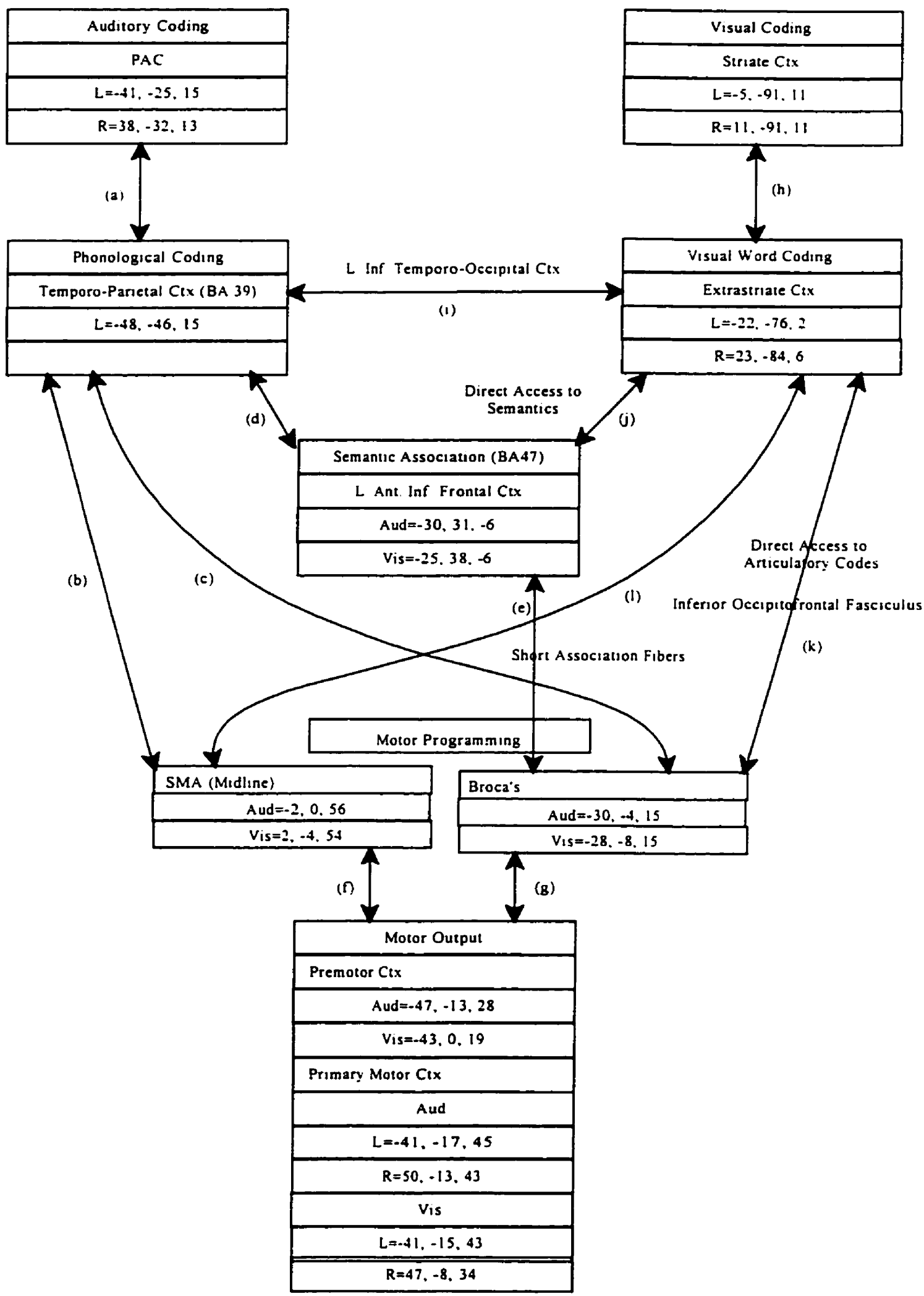

Figure 7 
case in the auditory stream, the visual code can be used to access both the semantic system and the output processing stages directly. An enhancement of the model was made by Petersen et al. (1989), in that a pathway connecting the temporoparietal cortex and the left medial extrastriate cortex was created, allowing for the conversion of auditory codes into visual word codes, and vice versa.

An examination of the stereotactic coordinates reveals bilateral activation during both initial auditory and visual coding stages, reflecting the fact that these activations are not tied to language-related processing. As expected, the temporoparietal activation, related to phonological coding was lateralized to the left hemisphere, although somewhat surprisingly, the analogous visual word coding stage exhibited a bilateral activation. The reason for this is unknown, particularly since other studies (Petersen et al., 1990; Price et al., 1996) have found left lateralized medial extrastriate activations during tasks in which words and pseudowords were presented. Activations noted during tasks of semantic association using both auditory and visual stimuli were found to be only $8.6 \mathrm{~mm}$ apart, indicating modality nonspecific processing. The same modality nonspecific finding can be noted for both the Broca's area and midline SMA activations, as well as all motor output related activations.

\section{Critique}

Petersen et al. successfully address one of the major drawbacks of most models spawned from within cognitive psychology, in that they extend their model by assigning specific neural substrates to each of the postulated functional modules. Assignment of substrate enables the model to make predictions with respect to lesion localization in clinical populations, thus drastically improving the clinical utility of this model over other cognitively-based models.

The most questionable assumption of this model stems from the postulated, and rather poorly defined, phonological coding stage. The model makes the somewhat adventurous claim that information is passed directly from the PAC to the inferior parietal lobe, where it undergoes language specific processing, i.e. phoneme extraction and lexical access. This would seem to be highly improbable given that numerous studies have pointed to the involvement of both the planum temporale and the posterior STG in 
language specific processing. Also, the model predicts that reciprocal connections must exist between the temporo-parietal area and both the left anterior inferior frontal cortex (BA 47) (semantic association) and Broca's area (BA 44) (motor programming). An examination of Figure 4 shows that, although no such connections have been found, BA 39 does have connections, albeit not proven reciprocal connections, with BA 46 and BA 6 , areas that fall close to the areas suggested by the Petersen et al. model. The above does not question the involvement of the temporo-parietal region in language processing, but merely attempts to point out that the model may assign too great a role to this region, while ignoring the contribution of adjacent regions.

This model also presumes the existence of two other pathways that should be considered. The first is the reciprocal pathway presumed to connect the phonological coding stage with its visual homologue. A connection of this type linking the left extrastriate cortex and the left angular gyrus via the left inferior temporo-occipital areas, has been proposed by Henderson (1986) and supported by cases of alexia without agraphia following left inferior temporo-occipital lesion, thus supporting the possibility of such a link. The model also requires a reciprocal connection between the extrastriate cortex and both BA 47 (semantic association) and BA 44 (motor programming). The superior longitudinal fasciculus seems unlikely to be the fiber tract used for the projection to both BA 47 and BA 44 , since its frontal projection connects primarily to dorsolateral prefrontal regions. Another possibility is presented by a combination of the inferior longitudinal fasciculus, which projects from the occipital cortex to the temporal pole, and the uncinate fasciculus, which connects the temporal pole with the inferior frontal gyrus. This solution would require a synapse at the temporal pole, which should result in an activation during studies in which visual stimuli are presented. Although temporal pole activations are rather uncommon during language studies, this could be the result of the activation being subtracted out, since baseline and activation tasks usually use the same modality. A third, and perhaps the most probable, option is the inferior occipitofrontal fasciculus, which projects from the frontal lobes to the temporal and occipital lobes without the need for a synapse. In summation, it would appear as if this connection, although not yet precisely defined, is indeed possible. 
Let us now turn to compare this model with two others. Examination of this model makes clear, a number of ways in which it differs from the clinically-oriented Geschwind model (1965), as well as from the cognitively-rooted model of Ellis and Young. The most striking difference from the Geschwind model is the redefinition of the angular gyrus from a critical cross-modal code conversion mechanism, to the module responsible for some rather vaguely defined phonological processing, as well as serving as the auditory input lexicon. The second rather striking difference from the Geschwind model is the inclusion of a frontal semantic association module, although the precise function of this module is not clearly defined. It is clear, however, that if one accepts Shallice's (1989) notion that a semantic system is comprised of two separable components; one responsible for access and control of the semantic store and one serving as the actual store of highly structured semantic information, Petersen et al. see this area as being a part of the former component. Finally, the Petersen et al. model differs from that of Geschwind in the high degree of specificity with which substrates have been assigned, avoiding the rather nebulous pseudo-areas encompassed by the terms "Broca's area" or "Wernicke's area".

Differences between the Petersen et al. model and that of Ellis and Young have also been noted. These differences can be summarized as follows: (1) the phonological coding stage of Petersen et al. is represented in the Ellis and Young model as two separate stages; a phoneme extraction stage, and an auditory input lexicon, (2) the consolidation of these two stages into one makes the unidirectional connection from the input to the output lexicon as proposed by Ellis and Young unnecessary, (3) the phoneme level stage of Ellis and Young is not explicitly accounted for in the Petersen et al. model; its proposed functionality must be considered as integrated into Petersen et al.'s motor programming stage, (4) Petersen et al. proposes a bi-directional connection between the phonological and visual word coding stages; a similar link exists in the Ellis and Young model, except that the link occurs at the level of the output rather than the input lexicons, and (5) the Petersen et al. model, by virtue of its use of PET, is much more specific in its assignment of substrate to postulated functions. Thus, the two models differ primarily in that the Petersen et al. model is a simplification of the Ellis and Young model with regard to the 
specification of functional stages, however, it exhibits much more specificity in the definition of substrate.

\section{Using PET to Test Models of Single-Word Processing}

Two models of language processing were presented in the immediately preceding paragraphs; the Geschwind model $(1965 ; 1969)$ and the model of Petersen et al. (1988; 1989). The Geschwind model still enjoys vast acceptance from clinical neurology, whereas the Petersen et al. model, or models like it, are gaining more acceptance from the area of cognitive neuroscience. We therefore find ourselves in a situation in which we are presented with two models, both of which have proven themselves to be eminently useful in their respective fields; the Geschwind model in the diagnosis of language disorders and lesion localization, and the Petersen et al. model, in attempting to supply a valid neuroanatomical foundation for cognitively-based models of language processing. Alas, even though both of these models have made significant contributions, both models, since they postulate different substrates for similar functions, obviously cannot both be right.

We thus find it necessary to propose a methodology that can be used to compare both of these models against each other in order to determine which of the models offer a "better fit to the data". Historically, the "data" that the models have had to fit has always been clinically derived, i.e. models needed to be able to account for the various symptoms associated with language disorders. Unfortunately, this approach, based on pathological findings, has the consequence of developing models that may not, as discussed previously, reflect the functioning of an unimpaired language system. This problem is compounded by the fact that both of the two proposed models have been developed with the pathological findings in mind, thus although both models show weaknesses and strengths, they both do a rather good job of accounting for much of the clinical data. One solution to this problem, the one to be further explored in the remainder of this paper, involves fitting the above mentioned models to PET data collected from a neurologically normal population during the performance of language tasks. 


\section{Experiment \# 1: Assigning Substrate to Functional Processes}

The prime advantages of using PET data to test models of language processing are (1) the ability to localize activation much more precisely than would be possible via the study of lesion data, and (2) the ability to test the models on a non-pathological population. The first step in the process of model testing via PET data requires us to precisely define the regions of interest (ROIs) that we believe to make a meaningful contribution to language. As mentioned previously, ROIs can be defined either anatomically via predefined templates, or physiologically based on previously done activation studies. As physiological ROI definition offers a number of advantages over the anatomical method, we shall opt for this method. Now, although the Geschwind model is clinically-based, and does define the proposed substrates, the degree of required specificity is lacking, since physiological ROIs are optimally defined as $x, y, z$ coordinate triplets in a stereotactic space, such as that of Talairach and Tournoux (1988). One could attempt to solve this problem by assigning to the Geschwind model substrates, the equivalent coordinates as determined by the Petersen et al. study. Unfortunately, this solution is not optimal, since, for example, Petersen et al. did not find an activation that accurately reflects the Wernicke area substrate that the Geschwind model requires. One also would need to assume that the activations of Petersen et al. are typical of all activation studies; an assumption that is not justified. It must also be noted that not all activation studies result in similar activations, even when an attempt is made at complete replication. Differences in activation locus may reflect differences in control tasks, activation tasks, brain morphology (particularly when only a very small number of subjects are scanned), preprocessing methods, methods of statistical analysis, or thresholding. As such, no one particular activation dataset can reliably be used to determine activation site loci, generalizeable to other datasets.

We are thus faced with the problem that, prior to being able to test our models, we must first be able to assign substrate to the various stages of the models that we intend to test. More specifically, we would like to be able to determine the locus of robust language-related activation sites, i.e. activation sites found to activate over a number of different language studies, carried out by a number of different laboratories. As such, it 
was decided that these substrates could best be determined by carrying out a metaanalysis of the PET language activation literature to date, and note those regions that most consistently activated between studies. The resulting physiologically-defined ROIs should then make it possible to assign specific substrates to any model that, itself, does not propose specific information with regard to the exact locus of the neural substrates associated with that model.

\section{Method}

Since the purpose of this experiment is to identify physiological ROIs from a review of language activation literature, one is confronted, once again, with the problem of determining an appropriate method to identify regions for inclusion into the metaanalysis. Studies which report their findings using gyral anatomy tend to lack specificity, i.e. an anterior middle frontal gyrus activation, while somewhat informative, is still quite vague. The problem is even worse for studies which report their findings in terms of pseudo-anatomical areas such as Broca's or Wernicke's areas, for reasons previously discussed. For these reasons, it was decided to sample only those studies that reported their findings using a standardized stereotactic space. This method was considered the most appropriate not only due to its degree of specificity with regard to localization, but also because the coordinates by which such activations are identified are more easily manipulated by statistical techniques.

\section{Criteria for Study Selection}

The focus of the literature search was on studies that found activations while subjects performed language related tasks. Language related tasks are rather broadly defined to include any task that requires the subject to perform any type of processing necessary to either produce, comprehend, or manipulate elements of language. Although it was realized that such a broad definition might result in the inclusion of a number of studies that would otherwise not be considered optimal, it was decided this potential drawback (1) was outweighed by the advantage of sampling over as many studies and conditions as possible, and (2) could be controlled for by the nature of the statistical analysis performed. 
The literature was sampled from 1988 to present, starting with the seminal study of Petersen et al. (1988), in which the subtractive methodology was first introduced. Earlier studies were avoided due to the fact that earlier studies (1) used quite low resolution scanners, (2) suffered from an imperfect ability to localize activations ( see Drevets et al., 1992), often due to the fact that many researchers used neither a stereotactic atlas nor a superimposed MRI to aid in localization, and finally (3) many of the earlier studies did not publish the stereotactic coordinates related to their activation sites.

\section{Statistical Analysis}

All activation $\mathrm{x}, \mathrm{y}$, and $\mathrm{z}$ coordinates were subjected to a $\mathrm{K}$-means cluster analysis, in order to produce anatomically meaningful clusters of activations. The K-means clustering was performed using the SPSS for Windows version 7 statistical software package. Input to the cluster analysis consisted of the $\mathrm{x}, \mathrm{y}$, and $\mathrm{z}$ coordinates for each activation data point, converted into the stereotactic space of Talairach and Tournoux (1988). Coordinates were not standardized, i.e. transformed to having a mean of 0 and a standard deviation of 1 , since all variables were measured on the same scale. The clustering process begins by assigning a given number of cluster centroid triplets, to which all other triplets are assigned. The initial centroids can either be explicitly assigned, if one is interested in hypothesis testing, or, as is currently the case, the centroids can be estimated from the data, if one is primarily interested in using the method to group the observations. Once the initial centroids have been computed, all other observations are then classified to one of the cluster centers, on the basis of Euclidean distance of a given coordinate to the cluster centroids, i.e. a case is assigned to the cluster for which the distance from the case to the cluster centroid is the smallest. Once all cases have been assigned, mean values for each variable $(x, y$, and $z)$ within each cluster are computed, resulting in new, adjusted cluster centers. If these new values do not differ significantly from the old, then the process stops, else the classification process repeats, using the new cluster centers. The final result should provide us with clusters of data points located very near to each other in physical space, since clustering was performed on measures of physical distance, i.e. the $x, y$, and $z$ coordinates measured in millimeters. 
Initial cluster analysis results were manually inspected with regard to determining the anatomical validity of each of the activations comprising each cluster. Activations that were found to be inappropriately clustered, e.g. an activation explicitly reported as being superior cerebellar being classified into an inferior occipital cluster, were either manually reclassified into another cluster, or removed from the analysis. Clusters were also examined with regard to the Euclidean distance of each cluster member from the cluster center. Target cluster size was defined to be such that no individual activation was to be more that $15 \mathrm{~mm}$ from the cluster center, thus creating clusters with a diameter of no greater than $30 \mathrm{~mm}$. Clusters found to be larger than the target size were, themselves, subjected to re-clustering to see whether the cluster could be broken into smaller, more tightly packed clusters. If this was not possible, outliers, identified as being located more than $15 \mathrm{~mm}$ from the cluster center, were removed from the cluster, and the cluster center coordinate was then recomputed. This procedure was repeated until the target cluster size was attained.

\section{$\underline{\text { Results }}$}

A search of the literature resulted in the sampling of 261 activations across 19 studies and 41 conditions. All activations were classified according to input modality, processing level, and task using the codes presented in Tables 1 and 2 . The results are summarized in Table 3.

Insert Table 3 about here

Of the initial 261 activations, 22 could not be clustered, and were thus removed from the analysis. The remaining 239 activations were grouped into 43 clusters. Figure 8 graphically displays cluster locations superimposed onto the left and right lateral surfaces of the rendered cortical surface of an averaged brain that was created as the result of averaging the individual MRIs of 305 neurologically intact individuals (Evans et al., 1992a). The medial activations are shown superimposed onto a blurred sagittal slice taken approximately $8 \mathrm{~mm}$ from the midline. Obviously, the displayed lateral and medial 
Table 3

Classification Summary Information

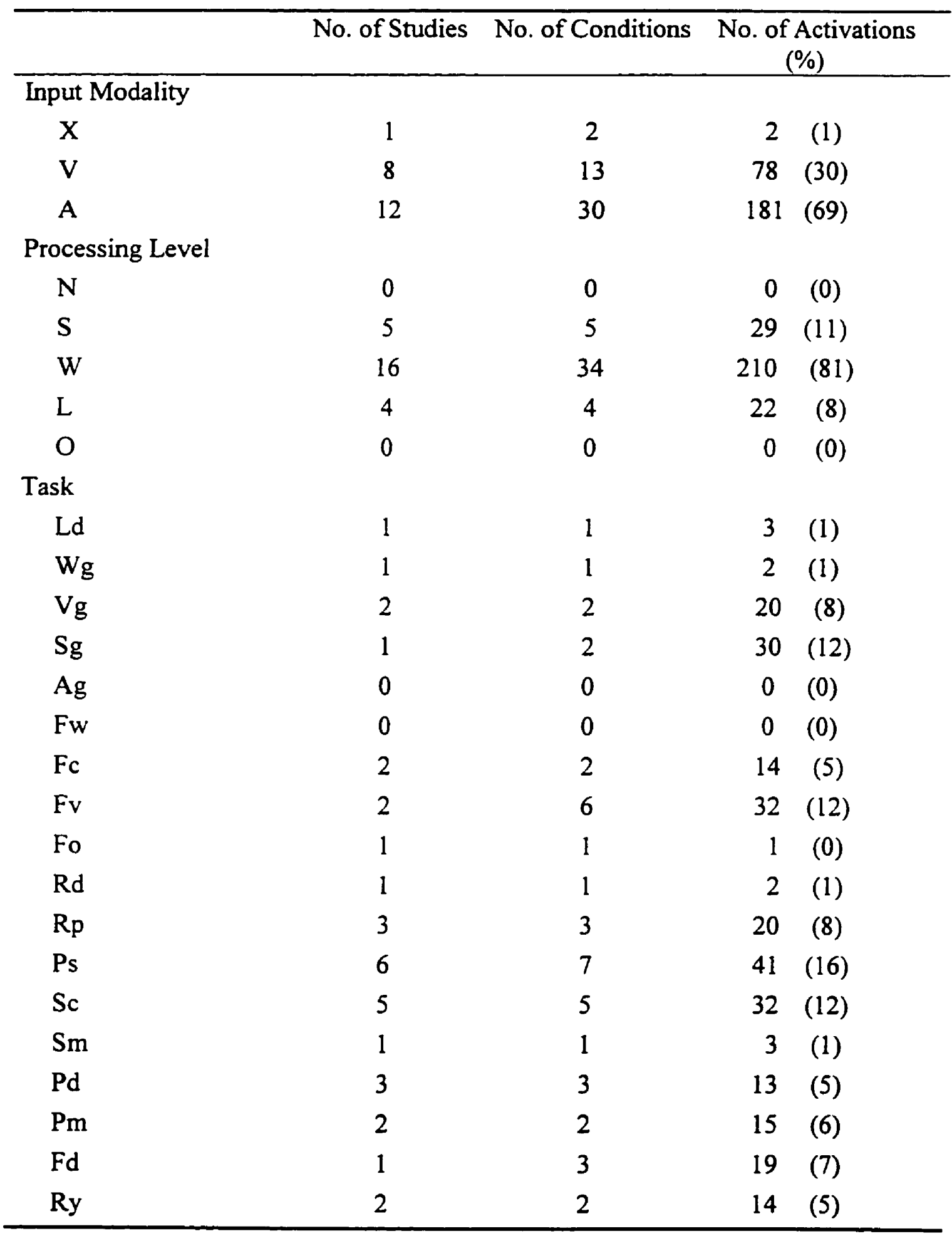


surfaces serve only as a reference frame, and cannot be used to extract detailed information about cluster localization. Details with regard to cluster size and location are presented in Tables 4 and 5. Although Tables 4 and 5 present information with regard to all clusters, the discussion to follow shall focus on those clusters that are of particular importance with regard to the task of assigning substrate to the Geschwind and Petersen et al. models.

Insert Table 4 about here

Insert Table 5 about here

Insert Figure 8 about here

\section{Frontal Lobe}

An examination of Figure 8 demonstrates a marked asymmetry of the frontal lobes with regard to both number of clusters and number of activations per cluster. Since language-specific frontal activations are typically lateralized to the dominant hemisphere, bilateral activations are thus indicative of either non-language processes, or articulatory processes. As such, all left-sided clusters were examined to see whether they might match any of the right-sided clusters in both location and function. The results are presented in Table 6. 
Table 4

Cluster Information - Frontal and Temporal Structures

\begin{tabular}{|c|c|c|c|c|c|}
\hline Cluster Name & $\begin{array}{l}\text { No. of } \\
\text { Points }\end{array}$ & $\mathrm{X}$ & $\mathrm{Y}$ & $\mathrm{Z}$ & $\begin{array}{l}\text { Description } \\
\text { (Talairach \& Tournoux, 1988) }\end{array}$ \\
\hline B11.47_L & 8 & -32 & 37 & -3 & BA $11 / 47$ \\
\hline B45.46_L & 11 & -42 & 29 & 14 & BA 45/46, MFS \\
\hline B4_L & 6 & -42 & -9 & 42 & BA 4 \\
\hline B45_L & 14 & -43 & 20 & 21 & BA 45, MFS \\
\hline B44_L & 10 & -41 & 9 & 28 & BA 44 \\
\hline B8.9_L & 3 & -27 & 41 & 31 & BA $8 / 9$ \\
\hline Bad01_L & 6 & -25 & -7 & $2 \mathrm{I}$ & Heterogenous cluster \\
\hline B47.In_L & 7 & -42 & 18 & 1 & BA $47 /$ insula \\
\hline Bad02_L & 5 & -26 & 21 & 5 & Heterogenous cluster \\
\hline Ins_R & 3 & 32 & 17 & 7 & Anterior insula \\
\hline B8.9_R & 2 & 34 & 29 & 34 & BA $8 / 9$, MFS \\
\hline B4_R & 2 & 48 & -11 & 39 & BA 4, PMC \\
\hline B37_L & 8 & -45 & -54 & -10 & BA 37, posterior ITG \\
\hline MTGr_L & 9 & -54 & -13 & -3 & BA 21, mid MTG \\
\hline B42_L & 10 & -53 & -24 & 9 & BA $41 / 42$ \\
\hline PT_L & 7 & -46 & -35 & 10 & BA $22 / 21$, STS or Planum Temporale \\
\hline MTSm_L & 9 & -46 & -37 & -8 & BA $21 / 20$, posterior MTS/ITG \\
\hline ITSm_L & 5 & -57 & -36 & -14 & BA $21 / 20$, posterior ITS \\
\hline B37_R & 3 & 37 & -58 & -5 & BA 37 , ITG basal surface \\
\hline STSr_R & 3 & 49 & 10 & -7 & BA 22, STS \\
\hline STSc_R & 5 & 52 & -35 & 7 & BA $22 / 21$, STS \\
\hline STSm_R & 6 & 52 & -15 & -4 & BA 21, STS \\
\hline PTr_R & 7 & 54 & -20 & 10 & BA $41 / 42$, or Planum Temporale \\
\hline PTc_R & 2 & 45 & -30 & 16 & BA 41, PAC \\
\hline PAC_L & N/A & -34 & -25 & 12 & Left Primary Auditory Cortex \\
\hline PAC_R & N/A & 38 & -23 & 7.5 & Right Primary Auditory Cortex \\
\hline
\end{tabular}


Table 5

Cluster Information - Parietal. Occipital. Medial. Cerebellar and Subcortical Structures

\begin{tabular}{llllll}
\hline Cluster Name & $\begin{array}{l}\text { No. of } \\
\text { Points }\end{array}$ & X & Y & Z & $\begin{array}{l}\text { Description } \\
\text { (Talairach \& Tournoux, 1988) }\end{array}$ \\
\hline B39_L & 8 & -48 & -54 & 21 & BA 39, posterior STS \\
B7.40_L & 6 & -36 & -58 & 41 & BA 7/40, Intraparietal sulcus \\
B40_L & 4 & -38 & -42 & 28 & Inferior anterior BA 40 \\
B19s_L & 5 & -21 & -77 & 4 & BA 17, PVC \\
B19i_L & 3 & -29 & -81 & -4 & BA 19, Fusiform \\
B18_M & 4 & -3 & -89 & 7 & BA 18, medial \\
B19_R & 4 & 17 & -84 & 13 & BA 17, PVC \\
B32.6_M & 10 & -5 & 11 & 50 & BA 32/6, SMA/cingulate \\
B8_M & 7 & -8 & 28 & 41 & BA 8 \\
B32r_M & 2 & -5 & 37 & 25 & BA 32 \\
B24_M & 6 & -1 & 15 & 26 & Posterior BA 24 CG \\
C01C_M & 5 & -1 & -68 & -22 & Cerebellum midline \\
C02r_M & 4 & -5 & -54 & -11 & Cerebellum midline \\
C01i_R & 3 & 19 & -85 & -31 & R. caudal cerebellum \\
C02lt_R & 3 & 37 & -64 & -26 & R. lateral cerebellum \\
C03d_R & 3 & 19 & -62 & -15 & R. cerebellum \\
Thal_L & 3 & -14 & -19 & 11 & L. lateral thalamus \\
MidB_M & 3 & 1 & -30 & -10 & Midbrain \\
Thal_R & 5 & 8 & -16 & 4 & R. medial thalamus \\
\hline & & & & & \\
\hline
\end{tabular}




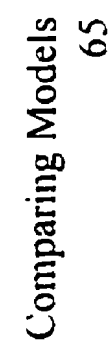
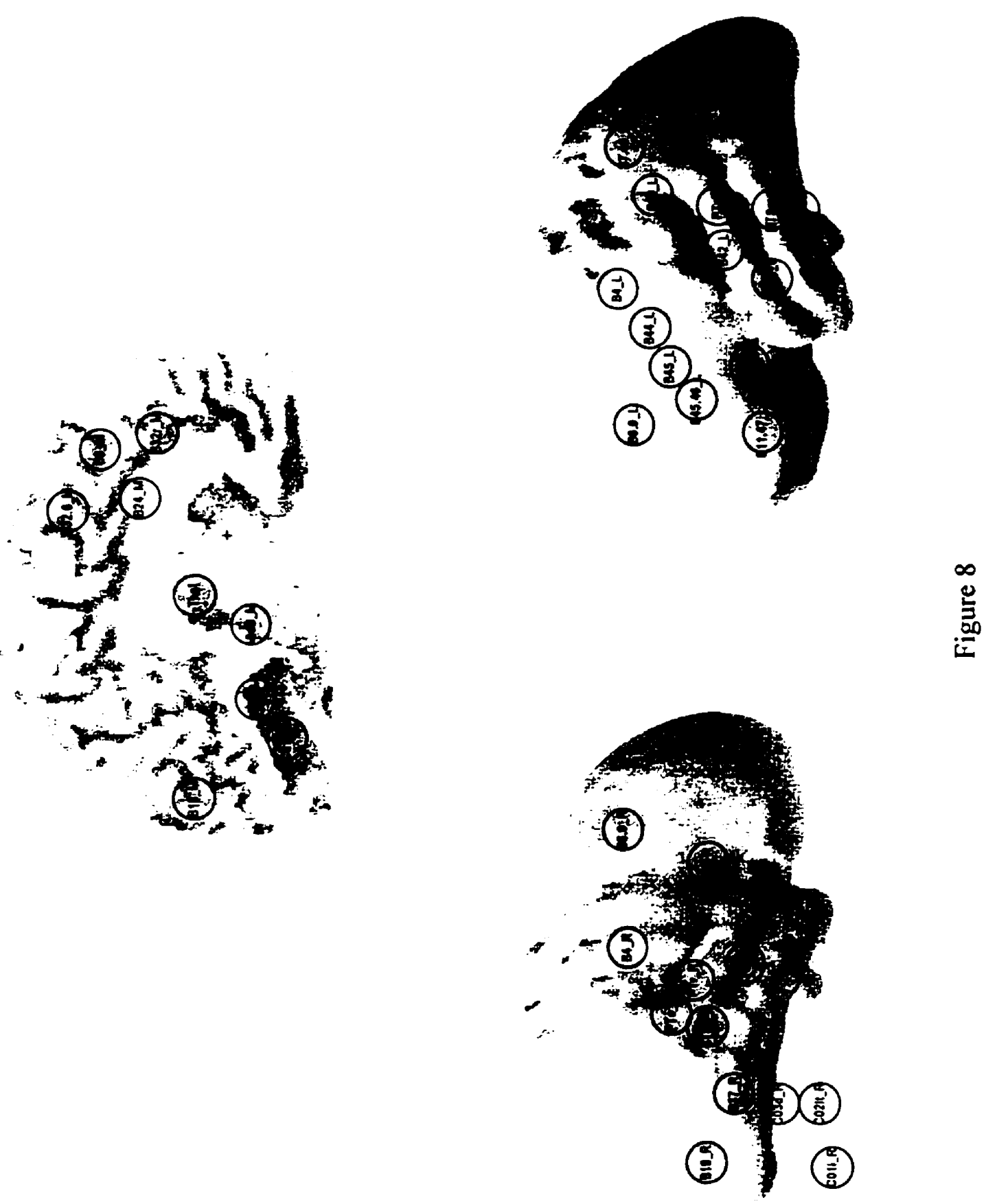
Bilateral Clusters. It should first be noted that Table 4 indicates that all three rightsided clusters are comprised of very few activations. In fact, 2 of the clusters consist of only 2 activations, a cluster size that would not have been accepted as a cluster on the left side. These right-sided clusters were, nonetheless, included in this analysis, since it was felt that they could still contribute to a better understanding of some of the left-sided clusters. As can be seen in Table 6, all 3 right-sided clusters were found to have homologous clusters on the left. Ins_R / B47.In_L are primarily involved in processing auditory stimuli, B8.9_R / B8.9_L both process visual and auditory word stimuli, and B4_R / B4_R seem to control the mouth area of the primary motor cortex.

Unilateral Clusters. The asymmetrical left-sided clusters are of great interest, since they reflect many of the language-related neuroanatomical findings discussed previously. As expected, clusters were found for BA 44 (B44_L), BA 45 (B45_L), and BA 11/47 (B11.47_L). B45_L differs from B44_L primarily in that (1) B45_L appears to have more fluency related tasks (verb, category, orthographic), and (2) B45_L tasks concentrate more on lexical retrieval and less on tasks requiring phonological processing. The processing profile of B11.47_L differs markedly from that of B45_L and B44_L in that (l) both visual and auditory stimuli are equally represented, whereas the other two areas favour auditory stimuli, (2) no fluency tasks are found in this cluster, (3) no phonological tasks are found in this cluster, and (4) most of the tasks are higher level tasks in which one response is generated for each stimulus, e.g. verb or synonym generation or semantic categorization. The last remaining cluster of interest is B45.46_L (BA 45/46), which somewhat unexpectedly formed a cluster separate from B45_L (BA 45). This cluster differs from B45_L in that it contains fewer fluency tasks (only 2 verb fluency activations), has even fewer phonological tasks, and seems to respond equally well to either visual or auditory stimuli. 
Table 6

Homologous Clusters

\begin{tabular}{llclll}
\hline $\begin{array}{l}\text { Right-Sided } \\
\text { Cluster }\end{array}$ & $\begin{array}{l}\text { Left-Sided } \\
\text { Cluster }\end{array}$ & $\begin{array}{c}\text { Inter-Cluster Distance } \\
(\mathrm{mm})\end{array}$ & \multicolumn{3}{c}{ Shared Characteristics } \\
\hline Ins_R & B47.In_L & 12 & Modality & Level & Task \\
B8.9_R & B8.9_L & 14 & A, V & W, L & Fv, Ps, Ry \\
B4_R & B4_L & 7 & A, V & W & Fc, Fd \\
B19_R & B19s_L & 12 & A, V & W & Rp \\
B37_R & B37_L & 10 & A,V & W & Ps, Pm \\
STSc_R & PT_L & 7 & V & S, W & Fd \\
STSm_R & MTGr_L & 3 & A & S, W & Fc, Rp, Ps, Sc \\
PTr_R & B42_L & 4 & A & S, W & Ps, Sc \\
\hline
\end{tabular}




\section{Temporal Lobe}

As was the case for the frontal lobe clusters, a number of temporal lobe clusters, with similar activation profiles, could be found in homologous regions of both hemispheres.

Bilateral Clusters. The pairing between homologous clusters PTr_R and B42_L are difficult to interpret, since both of these clusters encompass the region around the primary auditory cortex, thus allowing for the possibility of this area representing either PAC (BA 41 ), auditory association cortex (BA 42), or the planum temporale. A comparison of the PTr_R / B42_L cluster centers with the probability maps of the PAC (Penhune et al., 1996) and the planum temporale (Westbury et al., 1996), result in much higher probability values in the planum temporale $(\mathrm{p}=.50$ and $\mathrm{p}=.48$ ) than in the PAC $(\mathrm{p}=.10$ and $\mathrm{p}=.20$ ), indicating that these clusters might not primarily represent PAC. Additional support for the proposition that these clusters should not be interpreted as reflecting PAC is provided by the following distance related information: (1) an analysis of the Euclidean distances separating the PTr_R / B42_L cluster centers from the approximated center of the $\mathrm{PAC}$ probability map peaks (right $=47.5,-16.2,4.6$; left $=-42,-23.4,7.3$ ) is $9.27 \mathrm{~mm}$ and $11.15 \mathrm{~mm}$, and (2) the PTr_R / B42_L cluster centers are situated very close to the lateral surface, whereas BA 41 is situated more medially. As such, for the purposes of substrate assignment, PTr_R and B42_L shall be regarded as reflecting either the more laterally located auditory association cortex (BA 42) or the planum temporale.

Another somewhat less clear bilateral cluster pairing is that of B37_R / B37_L. The difficulty arises in that, although both clusters are involved in processing visually presented words and pseudowords during the performance of low-level feature discrimination tasks, B37_L is also involved in processing auditory stimuli during the performance of higher-level tasks, such as synonym generation, verb fluency, phonemic monitoring, and semantic categorization. The STSm_R / MTG_L cluster pairing has a somewhat less divergent activation profile, with both clusters involved in auditory word and pseudoword processing, although, whereas STSm_R activations are primarily word related, MTG_L $L$ activations reflect a greater emphasis on pseudoword processing. The final bilateral clister pairing, STSc_R/PT_L, is located in the posterior third of the STG, 
and is comprised of activations that result primarily from the auditory presentation of words during tasks which include semantic categorization and category fluency.

Unilateral Clusters. The two remaining left-sided clusters, MTSm_L and ITSm_L, are both located near the middle inferior temporal gyrus. These two clusters are only separated by $12.3 \mathrm{~mm}$ and share quite similar activation profiles, with both clusters favouring the auditory presentation of words, and engaged by tasks of fluency (verb and category), as well as generative tasks, such as verb or synonym generation. MTSm_L differentiates itself from ITSm_L in two respects: (1) MTSm_L is involved in tasks requiring a semantic component, such as monitoring or categorization, and (2) MTSm_L contains activations resulting from word repetition tasks.

Parietal Lobe

Only three parietal clusters were found, all left-sided. B39_L and B40_L, both of which are located at the inferior-most region of the inferior parietal lobe, seem somewhat more likely to activate in response to the presentation, either visually or auditorily, of pseudowords, whereas the more dorsally situated B7.40_L only contains activations resulting from the auditory presentation of words. With regard to task profiles, B39_L and B7.40_L are similar in that both clusters contain semantic-related activations, i.e. synonym generation, semantic categorization, or semantic monitoring, whereas B40_L contains primarily fluency-related activations.

Medial

All medial clusters share an involvement in lexical retrieval. The more caudally located B32.6_M appears particularly involved in fluency tasks, as well as other lexical tasks such as verb and synonym generation. Its involvement in fluency tasks is expected due to the proximity of B32.6_M to the SMA. B8_M, which is situated rostrally from B32.6_M, and thus further away from the SMA, while lacking the fluency activations, retains activations that result from generative and semantic categorization tasks. None of the medial clusters were found to have a preference for any particular presentation modality. 


\section{Discussion}

The primary purpose of this meta-analysis was to assign substrate to two of the models discussed previously: the Geschwind model, which suffered from an underspecification of the proposed language-related substrate, and the Petersen et al. model, which suffered from an over-specification of the substrate, since all substrates were generated post hoc, from only one dataset. The cluster analysis, as shall be described in greater detail below, was able to successfully generate new physiologically defined ROIs, that are both quite specific in their degree of localization, while at the same time being generalizeable to other datasets. We now turn to the task of assigning the new ROIs to each of the aforementioned models.

The Geschwind Model

Assigning clusters to the various components of the Geschwind model proved to be rather straight-forward (see Figure 9). The greatest difficulty involved assigning substrate to PAC. Although clusters PTr_R and B42_L fall quite close to the probability peaks as defined by Penhune et al. (1996), the rather low probability values for both cluster centers challenges the interpretation that these clusters represent PAC. As such, it would appear as if the most reasonable interpretation of these clusters would be as representing the para-auditory cortex as described in the second stage of the Geschwind model. This then leaves us with the problem of assigning substrate to PAC. At first glance, it would appear as if these regions would be best defined by using the peak values of Penhune et al. Unfortunately, the use of the peak PAC values would result in the overlap of the PAC sampling spheres with the spheres of adjacent temporal lobe clusters. It was therefore decided to shift the PAC sampling spheres somewhat medially in order to minimize the spatial autocorrelation problem. The final PAC coordinates, while maximizing the distance between PAC and adjacent sampling spheres (see Table 7), were adjusted in such a way so as to ensure that the PAC sampling spheres were still able to sample the PAC peak probability areas. The PAC coordinates are given in Table 4. 
Table 7

Inter-Cluster Distances Less than $20 \mathrm{~mm}$

\begin{tabular}{llc}
\hline Cluster A & Cluster B & $\begin{array}{c}\text { Euclidian Distance } \\
(\mathrm{mm})\end{array}$ \\
\hline B45_L & B44_L & 13.2 \\
PAC_L & PT_L & 15.75 \\
PAC_L & B42_L & 19.26 \\
PAC_R & STSc_R & 18.45 \\
PAC_R & PTr_R & 16.47 \\
B42_L & PT_L & 13.1 \\
STSc_R & PTr_R & 15.4 \\
\hline
\end{tabular}


Insert Table 7 about here

Insert Figure 9 about here

\section{The Petersen et al. (1988) Model}

Most of the problems encountered assigning substrate to the Petersen et al. model were similar to those encountered with the Geschwind model, i.e. assignment of substrate to the primary and secondary input cortices, and were solved in a similar fashion. Also in accordance with the substrate assigned to the Geschwind model, cluster B44_L was assigned the role of Broca's area, and clusters B4_L / B4_R were determined to represent the speech area of the primary motor cortex (PMC). Cluster B39_L, although included in both models, is assigned a slightly different role in the Petersen et al. model, in that it is implicated in a phonological coding function, rather than the cross-modal code conversion function foreseen by Geschwind. It should also be noted that although both models acknowledge the need for a processing stage between Broca's and the PMC, usually localized to either the SMA or premotor cortex, none of the models explicitly define the connectivity of such a stage, thus making cluster assignment rather difficult, even though an SMA cluster, B32.6_M, was identified. Since it was deemed critical to the integrity of the Petersen et al. model to assign this substrate, it was assumed, in accordance with the general framework of the Petersen et al. model, that processing flow proceeds in parallel from the phonological coding stage (the angular gyrus) to the semantic association area, Broca's area, and the SMA. A further parallel path was also then postulated to connect the PMC with both Broca's area and the SMA.. Further details with regard to inter-cluster connectivity, as proposed by the Petersen et al. model, are displayed in Figure 10. 


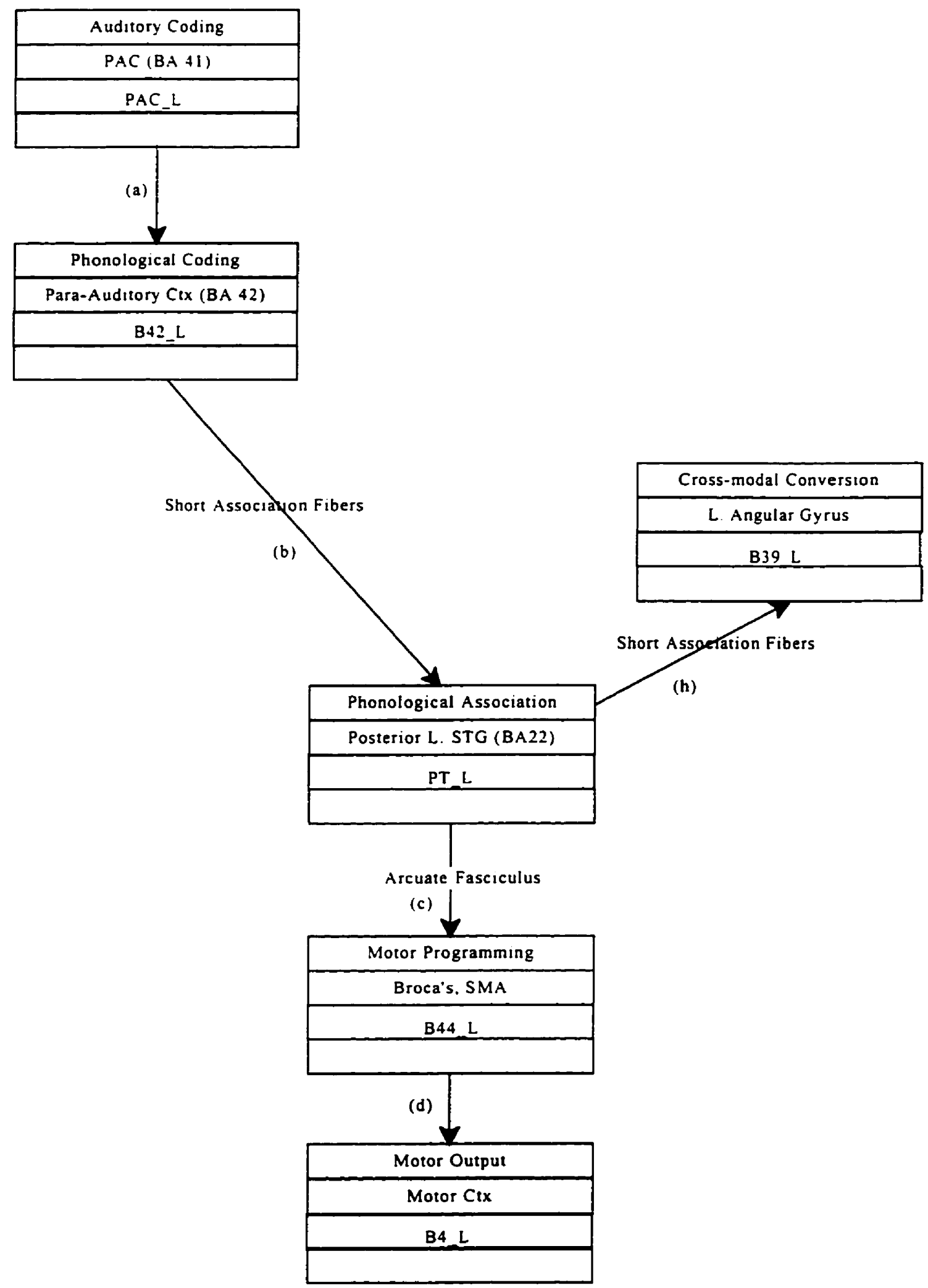

Figure 9 


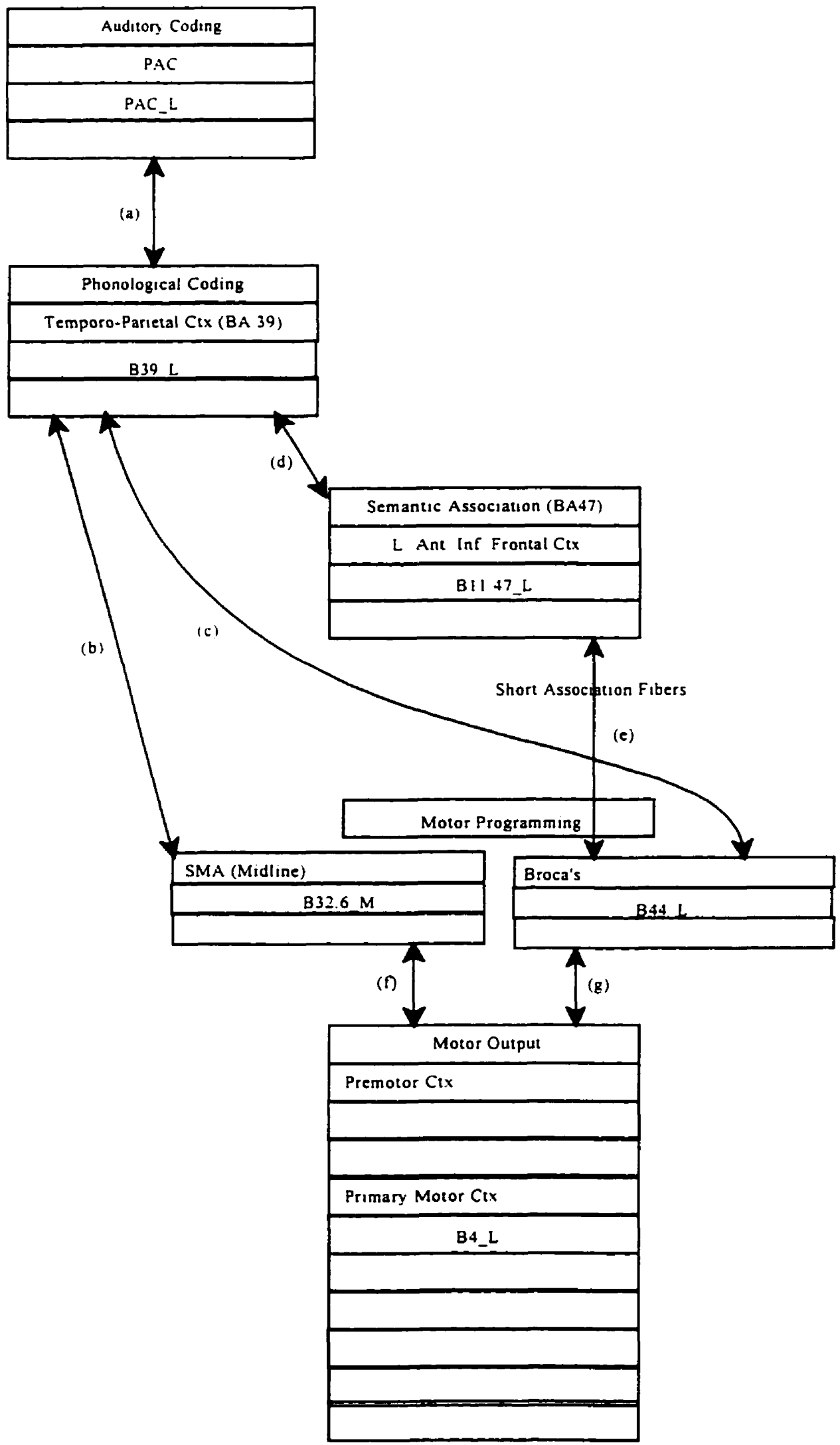

Figure 10 
Insert Figure 10 about here

\section{Experiment \# 2: Testing the Models}

Now that potential substrates have been assigned to the various stages of the models under consideration, the next step in this procedure is to judge the fitness of both of these models. This judgment can be accomplished at two different levels. Firstly, one can examine each model separately in order to see how it fits the data, and to determine whether minor adjustments, such as the addition of other neuroanatomically supported pathways might result in a better fit to the data. Secondly, the models can be compared against each other in order to see whether any one particular model fits the data better than the other.

In addition to the above mentioned model comparisons, this experiment will also seek to examine two somewhat different approaches to performing the required comparisons: (1) a method based on the examination of interregional correlation matrices, as demonstrated by Horwitz et al. (1992), and (2) a structural equation modeling approach (SEM), as demonstrated by McIntosh and Gonzalez-Lima (1992; 1993; 1994). Since both of the above methods are based on the analysis of either an interregional correlation matrix or an interregional covariance matrix, model tests could only be carried out on a dataset that was large enough to be able to generate stable interregional correlations. This problem was addressed by creating an aggregate dataset comprised of blood flow activation data derived from two separate studies conducted by Klein et al. (1995; 1996). Of greatest interest to the current study was the Klein et al. (1995) finding of a left inferior frontal gyrus activation, relative to the repetition baseline, during rhyme, and synonym generation tasks. Since this activation site, which was reported to be close to that reported by Petersen et al. (1988), was active regardless of whether the subjects were required to perform a phonological or a semantic search, the authors concluded that this 
region is associated with the process of general lexical search, rather than semantic processing, as was suggested by Petersen et al. (1988).

These particular datasets were selected, since the nature of the tasks used made them quite suitable to testing the assumptions of the Geschwind and Petersen et al. models. Both studies included a baseline condition in which subjects were simply asked to repeat a heard word, and an activation condition in which subjects were instructed to generate synonyms in response to auditorily presented words. The repetition task, due to its simplicity, made it possible to test many of the components included the Geschwind model, such as initial auditory coding, phonological coding, phonological association (lexical access), motor programming, and output. The simplicity of this task makes no explicit requirement for semantic access, which is ideal, since the Geschwind model does not claim to account for semantic processing. As such, one would expect the Geschwind model to be a better fit to the repetition data than the synonym generation data.

Conversely, the synonym generation task, due to its explicit requirement for semantic processing, allows us to test the more complex predictions embodied in the model of Petersen et al. The Petersen et al. model would predict that inclusion of the paths linking the semantic association substrate to the rest of the model, should not result in a significantly better fit when processing the repetition data, however, inclusion of these paths should contribute significantly to model fit when fitting the synonym generation data.

\section{Method}

\section{$\underline{\text { Subjects }}$}

Data for 19 subjects ( 9 male, 10 female) were obtained from a reanalysis of data collected by Klein et al. (1995; 1996). Mean age was determined to be 22.8 years. All subjects were right-handed and spoke English as their first language. Scanning protocols and procedures were in accordance with the ethical guidelines for human experimentation of the Montreal Neurological Institute.

\section{Scanning Methods}

Both PET and MRI scans were collected for all subjects. PET data were acquired using a Scanditronix PC-2048 system, yielding 15 slices at an intrinsic resolution of $5.0 \mathrm{X}$ 
$5.0 \times 6.0 \mathrm{~mm}$ (Evans et al., 199la). The tracer was introduced using the bolus $\mathrm{H}_{2} 15 \mathrm{O}$ methodology (Raichle et al., 1983), thus allowing regional CBF to be measured in each condition. Following acquisition, PET images were reconstructed using an $18 \mathrm{~mm}$ Hanning filter in order to account for individual differences in gyral anatomy, as well increase the signal to noise ratio. MR images were individually collected using a Philips 1.5T Gyroscan (63 slices, $2 \mathrm{~mm}$ thick), which were then coregistered with the PET images (Evans et al., 1991b). PET and MRI images were then linearly resampled into the standardized stereotaxic space of Talairach and Tournoux (1988) (Evans et al., 1992a), resulting in 80 transverse slices with voxel dimensions of $1.34 \times 1.72 \times 1.5 \mathrm{~mm}$. All volumes were then normalized to $100 \mathrm{~mL} / \mathrm{cm}^{3} / \mathrm{g}$ in order to remove the influence of global CBF (gCBF). This normalization step is of particular importance in studies of interregional correlations, since intersubject blood flow differences are greater than intrasubject differences, therefore performing a correlational analysis on raw CBF data would result in high correlations among all ROIs. After data was collected for all subjects, a final averaged MRI volume was created by averaging all of the resampled individual MR volumes.

\section{Stimuli}

The stimuli consisted of 2 lists of 22 words, presented binaurally through Eartone $3 \mathrm{~A}$ insert earphones. The word list used for the repetition task, although different from that used for the synonym generation condition, was matched to the synonym generation list with regard to word characteristics (word frequency, length, number of syllables, and part of speech).

\section{Testing Procedure}

Prior to scanning, earphones were inserted and subjects catheterized to allow for the administration of the bolus. Prior to each scan, subjects were instructed to keep their eyes closed. Each task was initiated 30 seconds prior to bolus injection. Scan acquisition was initiated automatically when the scanner detected radiation levels to be above a preset threshold level, and continued for 60 seconds thereafter. Stimuli were presented at a rate of one every 4.2 seconds. In the repetition condition, subjects had merely to repeat the presented word out loud, whereas in the synonym generation condition, subjects were 
required, within the allotted time, to generate a synonym corresponding to the presented word. If unable to generate a synonym, subjects were instructed to respond with the word "pass". Both latency and accuracy measures were recorded.

\section{$\underline{\text { ROI Definition }}$}

Both model comparison methods to be explored in this paper share the need to create either an interregional correlation matrix or an interregional covariance matrix. These matrices can only be produced by sampling the PET activation volumes at specific locations called regions of interest (ROIs). As discussed previously, ROIs can either be created a priori via the superimposition of an anatomical template upon the activation data, or post hoc, by creating ROIs at locations where activation peaks were previously found as the result of a statistical examination of the dataset, typically by inspecting the results of a subtraction. The current experiment steers a course between these two methods by using a priori defined physiological ROIs.

The results presented in experiment \#1 permit us to sample very specific locations from within our dataset, based purely on the findings of other language activation studies, whose activation coordinates have been cast into a standardized stereotaxic space. The creation of physiologically defined ROIs requires information with regard to the shape and the extent of the area to be sampled. It was decided to create spherical ROIs since spherical ROIs will result in a uniform sampling of the space around the centroid, and since spherical ROIs do not require us to impose assumptions about the precise nature of the ROI, as would be the case if one decided to shape the ROI to conform to the boundaries of a particular anatomical region. The next step is to define the precise extent of the ROI, or in the case of spherical ROIs, the radius of each sampling sphere. The extent of each sphere is constrained by two factors. Firstly, a sphere cannot be made too large, since, in doing so, one loses one of the greatest advantages of PET, i.e. the excellent spatial resolution. Secondly, one must be careful that one does not make a sphere so large so that it results in two adjacent spheres overlapping, and thus sampling the same voxels. The inclusion of the same voxels in different spheres will result in spatial autocorrelation, which manifests itself by artificially inflating the correlation between the sampling spheres. This potential problem is best illustrated in the current 
experiment by the cluster centroids for B44_L and B45_L. Table 7 shows that the centroids of these clusters are separated by $13.2 \mathrm{~mm}$. As such, the maximum radius for each of these sampling spheres must be no greater than $6.6(13.2 / 2) \mathrm{mm}$. The definition of a larger radius would necessarily require that some of the voxels located along the periphery of each of the spheres be included in both spheres, resulting in spatial autocorrelation.

In order to avoid this problem and thus reduce the effects of spatial autocorrelation, all intercluster Euclidean distances were computed (see Table 7), and the sampling radius for all ROIs was set to no greater than $1 / 2$ the distance separating the closest ROIs. In this case, it was decided that a $5 \mathrm{~mm}$ radius would be optimal. It should be noted at this point that this solution does not completely eliminate the effect of spatial autocorrelation, it simply reduces it. Spatial autocorrelation still will have a minimal effect, since our dataset has been blurred with an $18 \mathrm{~mm}$ Gaussian filter, and as such, voxels that are located near the periphery of any given ROI may still exert an influence onto the voxels of other nearby ROIs. Whether this impact is particularly strong is best determined by examining the strengths of the interregional correlations. Once the dimensions of the sampling spheres have been defined, actual activation data for each ROI is determined by computing which voxels fall within the defined sampling sphere for each individual ROI for each subject and condition. The final activation value for each ROI is simply calculated as the arithmetic mean activation of all voxels that comprise that $R O I$. Statistical Analysis

Interregional Correlations. Examination of the correlation matrices serve both exploratory and hypothesis testing purposes. A confirmatory analysis is made possible since both models make predictions with regard to the correlations that are expected to differ significantly from zero. With regard to the Geschwind model, an examination of Figure 9 demonstrates that only 5 correlations are predicted to be nonzero, i.e. paths (a), (b), (c), (d), and (h). Paths (e), (f) and (g) cannot be tested since our dataset does not include activation data in response to visually presented stimuli. Path $(\mathrm{h})$ running from the left posterior STG to the left angular gyrus can, however, be tested even in the absence of data resulting from visual stimulus presentation, since Geschwind explicitly 
states that auditory input can spread to visual areas via the angular gyrus. In order to test whether any of these correlations are, in fact, significant at the $\mathrm{p}=.05$ level, a Bonferroni correction for multiple comparisons must be applied. As such, correlations found to be significant at the $\mathrm{p}=.01(.05 / 5)$ level shall be declared as significant.

The significance levels for the correlations for the Petersen et al. model were for computed separately for each condition. As can be seen in Figure 10, removal of the purely visual areas from the model, leaves us with 7 paths (paths (a) through (g)) to be tested for significance. Application of the Bonferroni correction for multiple comparisons, lowers the alpha level from $\mathrm{p}=.05$ to $\mathrm{p}=.007(.05 / 7)$. Although this significance level is valid for the synonym generation data, it could be argued that such an alpha value is too conservative for the repetition data, since Petersen et al. envisioned the semantic association area (B11.47 L) to only be active during explicitly semantic tasks. As such, we should not be testing the correlations represented by paths (d) and (e), thus dropping the number of comparisons down to 5 . This, in turn would result in an adjusted alpha value of $\mathrm{p}=.01(.05 / 5)$ for the repetition data.

It is clear that in the course of model testing, some of the correlations will be found to be significant, as predicted, while others will fail to reach significance. These expected partial failures leave us with the opportunity of examining the remaining correlations in order to note whether gaps in the model under investigation might be filled through some other route. Such exploratory, hypothesis-generating work might yield in a slightly modified model, that could be tested in the future with a different dataset. However, rather then inspecting all of the remaining correlation coefficients, once must first limit the correlations to those that are statistically significant by controlling for multiple comparisons. This can be achieved, as was the case in the previous two paragraphs, by applying a Bonferroni correction to the experiment-wise alpha level, which is $\mathrm{p}=.05$ in this case. The number of comparisons would be computed by (1) calculating all possible correlations between the 13 sampled ROIs, and then (2) subtracting the number of comparisons that were made a priori (either 5 or 7 ). Dividing the experiment-wise error by the number of comparisons ( 73 or 71 ) results in a per comparison alpha of either 
$\mathrm{p}=.00068$ or $\mathrm{p}=.00071$, respectively. We shall use the value of $\mathrm{p}=.0007$ for these comparisons.

It should be noted at this point that, since we are dealing with rather small sample sizes, the calculation of the significance of the difference of a correlation coefficient, either from zero, or from another correlation coefficient, is somewhat more complex. In particular, although estimates of standard error are quite accurate when using a rather large number of observations, these estimates become increasingly less reliable as sample size decreases. This problem is best dealt with by estimating the standard errors from the sample itself via the use of a bootstrap procedure (Efron, 1981), as discussed in some depth by Horwitz et al. (1992). Although Horwitz et al. (1992) state that 100 bootstrap samples appear to generate stable estimates of standard error for correlation coefficients, a test of this recommendation with $\mathrm{rCBF}$ data found that the standard errors were actually most stable using 200 bootstrap samples (see Appendix A). As such, standard errors were estimated via bootstrap using 200 samples, and these were then in turn used to generate either t-values, in the case of tests comparing correlation coefficients to zero, or z-scores, when testing for a significant difference between two correlation coefficients.

Structural Equation Modeling. Structural equation modeling was performed with the aid of the AMOS 3.1 software package (Arbuckle, 1992), using maximum likelihood to generate all estimates. In order to increase the number of estimable parameters and keep all model error from being accounted for by residual variables, residuals were constrained following the suggestion of McIntosh and Gonzalez-Lima (1994). These authors argue that regions that receive input from other regions within the model (endogenous regions) should have their residuals fixed to between $35 \%$ and $50 \%$ of total region variance, with regions that receive a greater number of inputs, i.e. regions that have more predictors, allowing for less residual variance. Regions the receive no input from within the model (exogenous regions) must have their residuals set to $100 \%$ of their variance. As such, all ROIs, with the exception of PAC, had their residuals constrained to $50 \%$ of total region variance. PAC residuals were set to $100 \%$ in the Geschwind model, since PAC is exogenous in that model. Determination of PAC residual was somewhat more difficult for the Petersen et al. model, since this model allows for a feedback connection from 
temporo-parietal cortex. In recognition of the fact PAC most likely receives the bulk of its input from structures outside the model, it was decided to set the PAC residual to $70 \%$ of region variance, thus allowing for the feedback connection to exert some influence onto PAC.

Models were initially evaluated on a per model (Geschwind or Petersen et al.) and condition (repetition or synonym generation) basis, thus resulting in 4 models to be evaluated. The initial step in the evaluation process is the examination of the chi-square statistic for that particular model/condition, which forms the basis of an omnibus test of that model. The chi-square statistic can be interpreted as a goodness of fit measure, in that it is a measure of the error encountered when attempting to fit the sample data to the proposed model. Thus, a model that results in a high chi-square statistic indicatives a model that is a worse to fit to the data than one that produces a smaller chi-square statistic. The actual significance of the model is determined by computing a p-value based upon the chi-square statistic and a measure of model parsimony, as reflected by the model's degrees of freedom. The resultant p-value can be interpreted as "the approximate probability, under the hypothesis that the specified model is correct, that the chi-square statistic would have reached a value as high as its current value; thus if $\mathrm{p}<.05$, then the departure of the data from the model is significant at the $\mathrm{p}=.05$ level" (Arbuckle, 1992).

The goodness of fit of a model can also be described by the use of any number of goodness of fit indices. Most commonly used are termed (1) the goodness of fit index (GFI), and (2) the adjusted goodness of fit index (AGFI), both of which were proposed by Jöreskog and Sörbom (1984). The GFI is calculated by subtracting the ratio of sum of squares residual over sum of squares total (determined when comparing the sample covariance matrix to the estimated matrix) from 1 . The resultant index takes on values ranging from 0 to 1 , with 1 indicating a perfect fit. The AGFI is identical to the GFI, with a modification that forces it to take the parsimony of the model into account by adjusting the index for the number of parameters that must be estimated, i.e. the more parameters to be estimated, the more the index is adjusted downward. An AGFI of 1 indicates a perfect fit, a truly bad fit can result in a negative AGFI. Many structural equation modeling software packages will not only compute many different measures of fit, they will also do 
this for 2 defauit models: (1) the saturated model, and (2) the independence model. The saturated model is a version of the model which one is attempting to fit, with no constraints imposed, i.e. all parameters are free to be estimated. The second default model, the independence model, is the opposite of the saturated model, and much more useful. This model assumes that all paths between variables are fixed at zero, i.e. that all variables are uncorrelated. In doing so, the model will often be an extremely poor fit to most datasets, and thus the measures of fit of this default model will also reflect this poor degree of fit. The use of this default model is in that one can compare one's own models indices with that of the independence model. If the fit of the model under consideration has goodness of fit indices that are only minimally better than that of the independence model, the model under consideration might well require revision.

Following the test of the omnibus statistic and inspection of the various measures of fit in order to gain an appreciation for general model fit, one could next examine the actual path coefficients for the model in question to determine which of the coefficients were able to reach significance. Under the AMOS package, this is done by noting whether the critical ratio (CR), a measure formed by dividing the regression weight by the estimate of standard error, is above 2. The approximate value of 2 (actually 1.96) reflects the fact that the CR has a standard normal distribution, and therefore any CR greater than 1.96 would be significant at the $\mathrm{p}=.05$ level. The interpretation of the meaning of the path coefficient must be done carefully in blood flow studies. Simply, the path coefficient describes either the proportional increase or decrease in blood flow that would result from a 1 unit increase at the predictor variable, assuming all other variables are held constant. For example, if region A is linked to region B via a unidirectional link, with a path coefficient of, say, .4 , then, a 1 unit increase in blood flow at $A$ would result in a .4 unit increase at $B$. Similarly, if the path coefficient were -.4 , then a 1 unit increase at $\mathrm{A}$ would result in a .4 unit decrease at $\mathrm{B}$. Care must be taken not to interpret these coefficients as indicators of inhibition, as inhibition could well result in a rCBF increase.

Other model characteristics of interest include: (1) the modification indices, which suggest how the model might be improved by the consideration of additional paths, and 
(2) the squared multiple correlation (SMC), which indicates how much of the sample variance for a given ROI is accounted for by that ROI's predictors.

\section{$\underline{\text { Results }}$}

\section{Interregional Correlations}

The Geschwind Model. An examination of the correlation coefficients for both the repetition and synonym generation tasks as displayed in Table 8, demonstrates the existence of a great number of highly significant correlations (uncorrected for multiple comparisons). Somewhat surprisingly, only two of the correlations predicted to be significant by the Geschwind model, were found to be so: (1) path (d) (see Figure 9) linking left BA 44 to the left PMC was found to be significant in the repetition task, $\mathrm{t}=3.04, \mathrm{p}=.008$, and (2) path (a) representing the correlation between left PAC and BA 42 was found to be highly significant for the synonym generation task, $t=7.41, p<.0001$, and approaching significance in the repetition task, $t=2.45, \underline{p}=.025$. Path (b) linking BA 42 with ROI PT_L approached significance for both the repetition task, $\underline{t}=2.24, \underline{p}=.039$, and the synonym generation task, $t=2.06, p=.055$. An examination of Table 9 indicates that only the correlation between B42_L and B39_L was found to differ significantly from the repetition condition to the synonym generation condition, $\underline{z}=1.99, \mathrm{p}=.047$, with the correlation changing from $\underline{r}=-.08$ in the repetition task to $\underline{r}=.42$ in the synonym generation task. It should be noted, however, that Geschwind does not propose a link between these two areas.

Insert Table 8 about here

Insert Table 9 about here 
Table 8

Interregional Correlations for Repetition and Synonym Generation Conditions

\begin{tabular}{|c|c|c|c|c|c|c|c|c|c|c|c|c|c|}
\hline & B11.47_L & B45_L & B44_L & B4_L & B4_R & B32.6_M & PAC_L & PAC_R & B42_L & PT_L & PTr_R & STSc_R & B39_L \\
\hline $\mathrm{B} 11.47 \mathrm{~L}$ & & .07 & .03 & .11 & -.03 & .12 & .06 & .04 & -.24 & .14 & .13 & .00 & .24 \\
\hline B45_L & -.21 & & .58 \# & .13 & .22 & .12 & $-.51^{* *}$ & -.21 & -.31 & -.35 & $-.42 * \star$ & $.39 *$ & -.11 \\
\hline B44_L & -.24 & $.42^{*}$ & & .26 & .48 ** & $.41^{*}$ & $-.42 * *$ & .03 & $-.28 *$ & .16 & $-.36^{*}$ & .61 ** & -.02 \\
\hline B4_L & .1 & .31 & $.44^{* *}$ & & $.47^{* *}$ & .16 & -.10 & .08 & $-.34^{*}$ & .15 & $-.45^{* *}$ & .11 & -.26 \\
\hline PAC_L & .31 & -.40 & -.46 ** & $-.37 *$ & $-.38^{* *}$ & .00 & & $.35^{* *}$ & 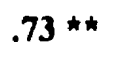 & $.53^{* *}$ & $.39^{* *}$ & -.22 & .24 \\
\hline PAC_R & .07 & -.16 & .35 & .11 & $.44^{* *}$ & -.24 & .14 & & $.43^{* *}$ & $.36^{* *}$ & $.57^{\text {* }}$ & $.54^{* *}$ & .41 \\
\hline B42_L & -.09 & -.28 & -.12 & $-.43^{* *}$ & $-.38^{* *}$ & .25 & $.50^{*}$ & .27 & & .44 & .27 & .11 & $.42^{*}$ \\
\hline B39_L & $.42 * *$ & .08 & .01 & -.07 & .19 & $-.49 * *$ & .18 & $.48^{* *}$ & -.08 & .16 & .28 & 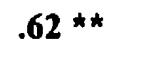 & \\
\hline
\end{tabular}

Correlations for the repetition condition are displayed below the diagonal, whereas those for the synonym generation condition are displayed above the diagonal.

$*=$ significant at the $\mathrm{p}=.05$ level (2-tailed)

$* *$ significant at the $\mathrm{p}=.01$ level (2-tailed)

Bold Faced $=$ significant at the $\mathrm{p}=.001$ level $(2$-tailed) 
Table 9

Differences in Interregional Correlations (Synonyn Generation - Repetition)

\begin{tabular}{|c|c|c|c|c|c|c|c|c|c|c|c|c|c|}
\hline & B11.47_L & B45_L & B44_L & B4_L & $\overline{B 4 \_R}$ & B32.6_M & $\overline{\text { PAC_L }}$ & PAC_R & B42_L & $\mathrm{PT}_{-} \mathrm{L}$ & $\overline{\text { PTr_R }}$ & STSc_R & B39_L \\
\hline \multicolumn{14}{|l|}{ B11.47_L } \\
\hline B45_L & .28 & & & & & & & & & & & & \\
\hline B44_L & .27 & .16 & & & & & & & & & & & \\
\hline B4_L & .01 & -.17 & -.18 & & & & & & & & & & \\
\hline B4_R & -.03 & -.21 & -.16 & -.16 & & & & & & & & & \\
\hline B32.6_M & -.07 & .29 & .22 & -.11 & -.04 & & & & & & & & \\
\hline PAC_L & -.25 & -.11 & .03 & .28 & $.30^{*}$ & .00 & & & & & & & \\
\hline PAC_R & -.03 & .05 & -.32 & -.04 & .19 & .05 & .21 & & & & & & \\
\hline B42_L & -.15 & -.03 & -.16 & .09 & $.50^{*}$ & -.31 & .22 & .16 & & & & & \\
\hline PT_L & -.07 & .01 & .02 & .07 & .29 & -.11 & .03 & .03 & .00 & & & & \\
\hline PTr_R & .24 & -.23 & -.32 & -.20 & -.14 & -.08 & $.51 *$ & .00 & .20 & .12 & & & \\
\hline STSc_R & .03 & .12 & .23 & -.03 & $.26 * *$ & .17 & -.09 & -.16 & .17 & .04 & -.33 & & \\
\hline B39_L & -.18 & -.20 & -.03 & -.19 & -.05 & .27 & .06 & -.06 & $.50^{*}$ & .14 & .18 & -.15 & \\
\hline
\end{tabular}

$\bullet=$ significant at $\mathrm{p}=.05$ (2-tailed)

$* *=$ significant at $\mathrm{Q}=.01 \quad$ (2-tailed) 
The Petersen et al. Model. An examination of the correlations for the Petersen et al. model, shows this model to fare little better than the Geschwind model. Only two correlations were found to be significant at the Bonferroni corrected alpha level. These correlations, which both occurred only in the repetition condition, included: (1) path (b) (see Figure 10), connecting the angular gyrus to the SMA, $\underline{t}=3.49, \mathrm{p}=.0028$, and as was the case for the Geschwind model, (2) path (g), connecting BA 44 to the left PMC, $\underline{t}=3.04, p=.0075$. All other correlations for either the repetition or the synonym generation conditions did not even approach significance, with the exception of path (d), connecting the angular gyrus to BA 11/47. Interestingly, even though Petersen et al.'s model quite explicitly denies any possible contribution of this area during a simple word repetition task, path (d) does seem, nonetheless, to make a contribution, $t=3.80, p=.001$. However, since this path was not explicitly tested a priori, its significance can only be tested using the global Bonferroni correction, i.e. the correction assuming 73 comparisons, at which level path (d) fails to find significance.

\section{Structural Equation Modeling}

The Geschwind Model. The omnibus tests of the Geschwind model found that this model could be rejected as being an acceptable fit to the data for both the repetition, $\chi^{2}$ $(16)=35.1, \mathrm{p}=.004$, and the synonym generation, $\chi^{2}(16)=33.5, \mathrm{p}=.006$, conditions. Examination of the critical ratios for both conditions, found results similar to those found via the interregional correlations approach. Reaching significance in the repetition task at the $\mathrm{p}<.05$ level, were paths (a), $z=3.02$, and (b), $\underline{z}=2.26$ (see Figure 11). Both paths (c) and $(\mathrm{h})$, which, according to the model, project efferent fibers that originate from the phonological association area, did not approach significance. The results for the synonym generation task were similar, with paths (a) and (b) reaching significance, with $\mathrm{z}$-scores of $\underline{z}=4.37$ and $\underline{z}=2.68, \underline{p}<.05$, respectively. As was the case with the interregional correlations, path (d) linking BA 44 with PMC was clearly not significant, $\underline{z}=1.11$ ). 


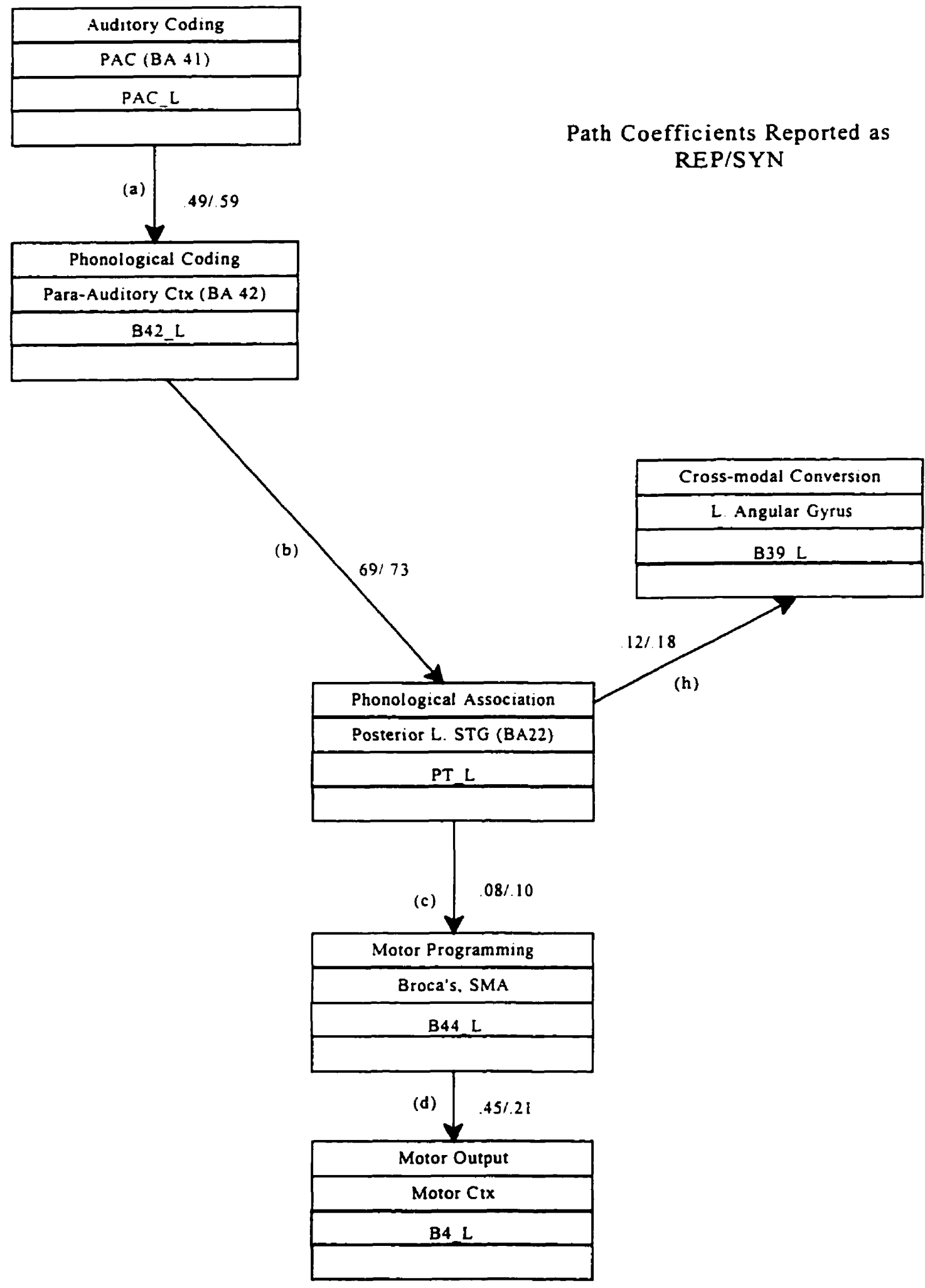

Figure 11 
The path linking PAC_L to B42_L was then further explored, since a certain amount of confusion was encountered with regard to this path in the interregional correlation analysis, i.e. why was it stronger in the synonym generation task than in the repetition task? As previously mentioned the link from PAC_L to B42_L was found to be significant. Next, the existence of a reciprocal link was tested. The PAC_L residual was set to $70 \%$ of total variance, and an additional path, linking B42_L to PAC_L was installed into the model and initially set to zero. The model was run and the PAC_L to B42_L path was still found to be significant, $\underline{z}=3.66, \mathrm{p}<.05$. The parameter on the new path was then freed and the model rerun. As a result of the additional path, the old path coefficient dropped slightly, $\beta=.472$, however, remained significant, $\underline{z}=2.60, \underline{\mathrm{p}}<.05$. The new path was not found to contribute significantly to the fit of the model, $\Delta \chi^{2}=1.32, \Delta$ $\mathrm{df}=1, \mathrm{p}=.25$, nor was it, itself, significant, $\underline{z}=1.07, \mathrm{p}>.05$.

The squared multiple correlations (SMC) generally reflected the weakness of the model. The highest SMC was found for ROI B42_L, for which 33.6\%, for repetition, and $51.5 \%$, for synonym generation, of its variance was accounted for by its single predictor variable, left PAC. Second best predicted ROI was PT_L, which had $22.1 \%$ and $28.5 \%$ of its variance accounted for in the repetition and synonym generation tasks by its single predictor variable, B42_L. Also of interest is the SMC of B4_L, that dropped from $16.6 \%$ in the repetition task to $6.5 \%$ in the synonym generation task, reflecting a change in processing occurring at its predictor variable, B44_L, during the synonym generation task.

Inspection of the modification indices reveals that the data for both tasks would fit the model better, if a temporo-frontal path were to be added to the model. In particular, both tasks would benefit from the inclusion of a link from PAC_L to B44_L. The addition of such a path, which would take on a path coefficient of approximately -0.45 , would result in a drop in the $\chi^{2}$ statistic of between 9.2 and 9.9 at the cost of only one degree of freedom (significant at the $\mathrm{p}<.005$ level). Whether the physiology supports such a modification shall be discussed in the next section.

Although not predicted by the Geschwind model, the results of the interregional correlation analysis, suggested that a similar analysis as the above also be performed 
using the right hemisphere homologous ROIs. Of greatest interest was that, although the omnibus test of the model to the repetition data found the model to be an adequate fit, $\chi^{2}$ $(16)=21.56, \mathrm{p}=.158$, the same test of the synonym generation data resulted in a clear rejection of the model, $\chi^{2}(16)=52.74, \mathrm{p}<.001$. This finding is of particular interest, since the results of the interregional correlation found there to be very strong and highly significant correlations amongst most of the right hemisphere ROIs included in the Geschwind model. The primary factor responsible for the rejection of the model for the synonym generation data appears to be the very weak and noisy path (b), linking the planum ROI PTr_R with ROI STSc_R, located in caudal superior temporal sulcus. Left hemisphere analyses assigned path coefficients of .690 and .729 to this path for the repetition and synonym generation tasks, respectively. As discussed previously, both of these coefficients were found to be significant at the $\mathrm{p}=.05$ level. Similarly, the rightsided repetition-related path coefficient was also found to be significant, $\underline{z}=2.76, \underline{\mathrm{z}}<.05$, and of similar magnitude, $\beta=.770$. The magnitude of this path coefficient was found to decrease in the synonym generation condition, $\beta=.215$, and was no longer found to differ significantly from zero, $\underline{z}=.933, \mathrm{p}>.05$. With regard to noise, inspection of area PT_L SMCs (area PT_L is the left-sided homologue to STSc_R), found them to be moderately high and of similar magnitude between conditions ( $22.1 \%$ for repetition, $28.5 \%$ for synonym generation). The STSc_R SMC for the repetition condition was also found to be in the same range as for PT_L $(29.7 \%)$, however, in the synonym generation condition the SMC decreased by approximately $85 \%$ to $4.6 \%$.

Examination of the modification indices for the repetition task suggests the addition of a path from PAC_R to STSc_R, resulting in a minimum decrease in the omnibus $\chi^{2}$ statistic of 5.907 at the expense of 1 degree of freedom. Although this modification would be marginally significant at the $\mathrm{p}=.05$ level, a physiological basis for such a modification is not clear. The modification indices for the synonym generation task reflect the poor fit of the model to the sample data, by proposing the possible addition of three temporo-frontal connections, each significant at the $\mathrm{p}=.05$ level. Clearly, however, one would do best to formulate a new model to embrace the results of the synonym generation data, rather than attempting to incorporate these post hoc suggestions. 
The Petersen et al. Model. The results of the testing of the Petersen et al. model were not encouraging. The fit of the model to the repetition data was tested by fixing the path coefficients for paths (d) and (e) (see Figures 12 and 13) to zero. Fixing these paths to zero in both directions, thus isolating the ROI representing the semantic association area from the rest of the model, reflects the prediction of the Petersen et al. model, that this area should not make a significant contribution to a non-semantic task, such as that of simple repetition. Computation of the omnibus test statistic indicated that the model was a particularly bad fit to the data, $\chi^{2}(11)=35.0, \underline{p}<.001$. This was reflected by the fact that none of the critical ratios for any of the proposed paths were able to attain significance at the $\mathrm{p}=.05$ level.

Insert Figure 12 about here

Insert Figure 13 about here

Inspection of the modification indices, suggested that the model would benefit from the inclusion of a path linking B11.47_L to B39_L, resulting in a minimum drop in the $\chi^{2}$ omnibus statistic of 15.6 at the expense of one degree of freedom. The second highest modification index proposed the addition of a reciprocal path from B39_L to B11.47_L. Since these linkages actually comprise part of the Petersen et al. model, it was decided to rerun the model, while estimating, i.e. freeing, the path coefficients for paths (d) and (e). The omnibus statistic for the modified model, although still indicating that the model be rejected, $\chi^{2}(7)=21.8, \mathrm{p}=.003$, did find that freeing the new paths contributed significantly to the fit of the model, $\Delta \chi^{2}=13.2, \Delta d f=4, \underline{p}<.025$.

Attempting to fit the model to the synonym generation data resulted in similarly poor results as encountered for the repetition data. The full Petersen et al. model, which included the paths required to link the semantic association area, ROI B11.47_L, into the 


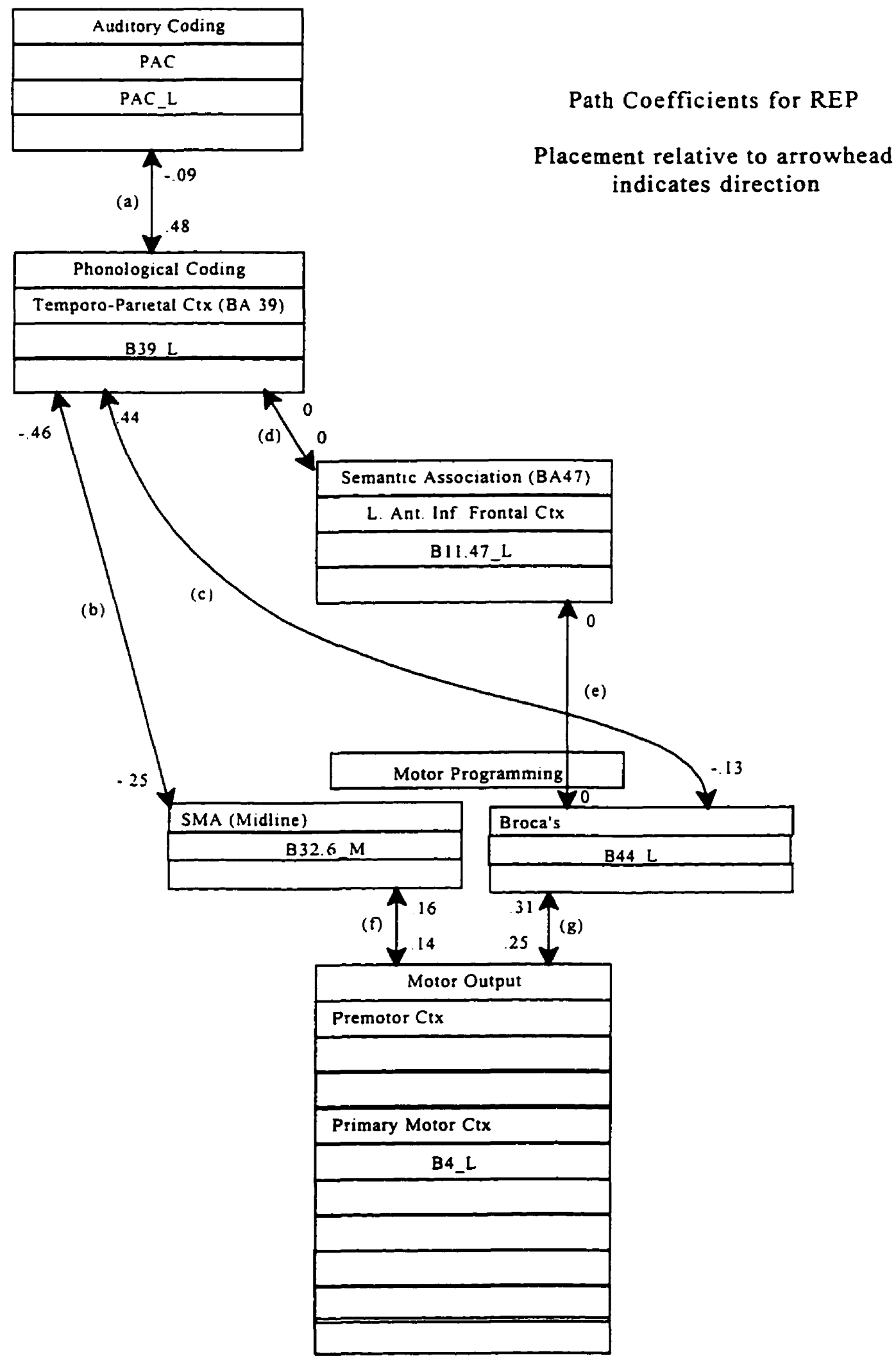

Figure 12 


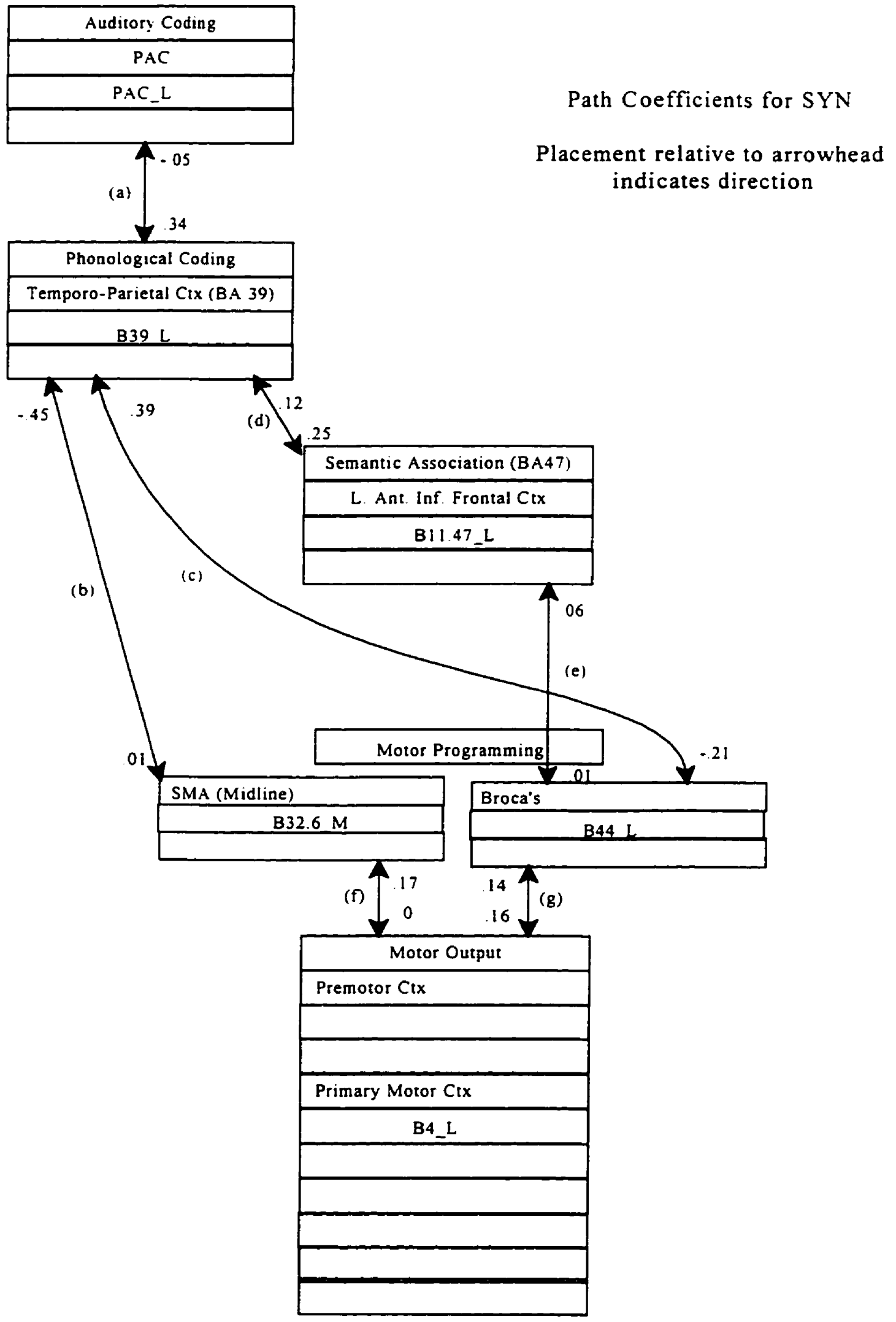

Figure 13 
model, provided an astonishingly poor fit to the data, $\chi^{2}(7)=31.2, \mathrm{p}<.001$. Also as was the case for the repetition data, none of the critical ratios for any of the paths were found to even approach significance. The devastatingly poor fit of the model, precludes the examination of any of the other conventional indices of fit. It is of interest, however, that although the AGFI for the Petersen et al. model was .100, the AGFI for the independence model was a rather high 0.731 . This result suggests that even if one were to attempt to modify the model to fit the sample data, one would still have great difficulty in achieving a better goodness of fit than can be found by simply assuming that all of the areas are functionally independent of one another. This would argue that, with regard to synonym generation, the problem with the Petersen et al. model is not only that required pathways may be missing, but that the model is missing the areas that are critical to synonym generation.

As a final test of one of the Petersen et al. model's assumptions, the paths linking the semantic association area were fixed at zero, and the model rerun. The results were similar to those found with the repetition data in that, as expected, the model was still rejected at any conventional level of significance, $\chi^{2}(11)=34.3, \mathfrak{p}<.001$. Of interest, however, was the fact that fixing these paths, and thus removing the effect of the semantic component from the model, did not result in a significant decrease in the fit of the model, $\Delta \chi^{2}=3.1, \Delta \mathrm{df}=4, \mathrm{p}>.5$. This casts yet more doubt on the involvement of B11.47_L in tasks of semantic association.

Model Comparisons. Examination of the GFI and AGFI measures of fit reflect the results of the various omnibus tests. The best model was found to be that of Geschwind, when imposed onto the right hemisphere and used to fit the repetition data (GFI $=.729$, AGFI =.644). This model was followed by the left hemisphere Geschwind model for the repetition data $(\mathrm{GFI}=.688, \mathrm{AGFI}=.591)$ and for the synonym generation data $(\mathrm{GFI}=.654$, AGFI $=.546$ ), however, as previously noted, this model was rejected as an appropriate fit to the data by the omnibus tests. The Petersen et al. model was an abysmal fit for the repetition data ( $\mathrm{GFI}=.649, \mathrm{AGFI}=.330$ ), and an even worse fit for the synonym generation data (GFI=.700, AGFI=.100). As mentioned previously, it is of note that the fit of the 
Petersen et al. model to the synonym generation data is substantially worse than that provided by the independence model $(\mathrm{GFI}=.808, \mathrm{AGFI}=.731)$.

\section{Discussion}

\section{Interregional Correlations}

Examination of the interregional correlations brings into rather sharp focus one of the limitations of the interregional correlations approach, i.e. that when looking at correlations, we are unable to test specific hypotheses about the directionality of proposed linkages. For example, the interregional correlational analysis of the Geschwind model (See Figure 9) found path (a) linking left PAC with left BA 42 to be highly significant during the synonym generation task, yet nonsignificant during the repetition task. How is this to be interpreted? ROIs representing primary sensory and motor cortices were purposely included in all models, since any areas that were found to correlate highly with them could be said to be engaged in lower level processing. With this in mind, one would have to interpret the path (a) correlation by claiming that BA 42 is more involved in low level sensory processing during a synonym generation task than during a repetition task. Yet, this assertion is highly counter-intuitive. The problem is a result of the fact that the correlation does not reflect directionality, although the model does. As such, rather than assuming the model to be correct with regards to directionality, one could just as rightly assume the model to be incorrect, since it makes more intuitive sense that BA 42 influences left PAC (perhaps in an inhibitory way) during the synonym generation task, in order to squelch unnecessary auditory input, which might otherwise shift attention from the higher level processing task. This problem exists in all correlational analyses, and thus requires the researcher to be very careful when attempting to interpret the results. Causality (and thus directionality of proposed linkages) cannot be tested by purely correlational methods.

Having noted the above potential problem, we now turn to considering how well the data fit the Geschwind model. Considering that only 2 of the 10 interregional correlations predicted by the Geschwind model were found to be significant, it would appear as if the data do not support the Geschwind model particularly well. This lack of support is exacerbated by the fact that: (1) the significant correlation found at path (a) for the 
synonym generation task (see Figure 9) is not, as noted above, easily interpreted, and (2) the path (c) linking the anterior speech area with the posterior speech area, a critical assumption of the Geschwind model, did not approach significance for either task. Only path (d) linking left BA 44 with left PMC was confirmed by the data and can be relatively easily interpreted. Since both of these areas were significantly correlated for the repetition task, but not the synonym generation task, this points to the fact that the processing profile of BA 44 changes with the task, i.e. during the repetition task, BA 44 must have been performing lower level motor programming related tasks, whereas for the synonym generation task, the nonsignificant correlation with $\mathrm{BA} 4$ indicates the involvement of BA 44 in some other higher level task that may be related either to lexical access or phoneme assembly.

Although the above does not abide well for the Geschwind model, examination of the interregional correlations for the right-sided homologous ROIs provide some interesting information. The reader should note that the right-sided homologous ROIs were created as a result of the same literature review that was used to create the left-sided clusters. Clusters were defined to be homologous if they shared a task profile and if they were very close in proximity (with correction made for hemisphere). The initial test of the Geschwind model used only left-sided ROIs, since Geschwind was quite clear that his model was a model of language processing in the dominant hemisphere, which is, of course, the left hemisphere for the vast majority of individuals. An inspection of the interregional correlations that were found to be significant even after the application of a global Bonferroni correction adjusting for 73 comparisons (see Table 10) is of particular interest due to the predominance of right-sided ROIs. Yet more fascinating is the fact that the right-sided interregional correlations are extremely supportive of the Geschwind model. With reference to Figure 9, and using the right-sided homologous areas, it can be seen that: (1) path (a) exists for both conditions, (2) path (b) does not exist, however, an alternative path linking right PAC to the PT_L homologue (STSc_R) takes its place, (3) the critical link (c) exists, (4) path (h) providing access to the angular gyrus exists, and (5) path (d) linking left BA 44 to right PMC also exists. All right-sided correlations that fall within the Geschwind model were also noted to be higher than their left-sided counter- 
parts. Other right-sided paths may have been found significant, had we not had to perform a global Bonferroni correction. A possible explanation for this finding is presented below.

Insert Table 10 about here

The Petersen et al. model (see Figure 10) appears to be just as incongruous with the observed pattern of left-sided interregional correlations as the Geschwind model. Path (a) linking left PAC to the temporo-parietal cortex (B39_L) did not approach significance in any of the conditions. This was to be expected, since it is known that efferent PAC fibers project primarily only to immediately adjacent association cortex in higher-order primates (Flechsig's principle). Paths (c) and (e) also failed to achieve significance for both conditions, thus leaving no plausible pathway to transmit information from the posterior speech area to the anterior speech area, effectively isolating BA 44 from any input. One of the few significant correlations observed was that of path (b), linking temporo-parietal cortex with SMA, during the repetition task. However, this correlation is difficult to interpret, since all other correlations either defining the linkage between temporo-parietal cortex and PAC or SMA and PMC were nonsignificant. An attempt to fit the model onto the right hemisphere, a tactic that proved profitable for the Geschwind model, failed, since none of the areas, other than the primary receiving or output areas, had right-sided homologous ROIs. As such, the model of Petersen et al. can be said to have fared much worse than that of Geschwind.

Structural Equation Modeling At a macroscopic level, the results of the structural equation modeling (SEM) of the Geschwind model were very much in accord with those attained via the interregional correlations (IRCs). The SEM approach rejected the Geschwind model as a reasonable fit to the data under all conditions, other than when fitting the right hemisphere repetition data. This result reflects that attained by the IRC approach, if one makes the assumption that the model must be rejected unless all critical pathways are found to be significant. As was previously noted, this was not the case for 
Table 10

Significant Interregional Correlations Not Predicted by Either Model

\begin{tabular}{llllll}
\hline Condition & ROI A & ROI B & Pearson's r & t-stat & p-value \\
\hline REP & B44_L & B4_R & .63 & 7.53 & $<.0001$ \\
& B4_L & B4_R & .63 & 5.40 & $<.0001$ \\
& PAC_R & PTr_R & .57 & 5.11 & .0001 \\
& PAC_R & STSc_R & .70 & 8.80 & $<.0001$ \\
& STSc_R & B39_L & .62 & 5.07 & .0001 \\
SYN & & & & & \\
& B45_L & B44_L & .58 & 4.74 & .0002 \\
& B44_L & B4_R & .48 & 4.81 & .0002 \\
& B44_L & STSc_R & .61 & 5.89 & $<.0001$ \\
& B4_R & PAC_R & .63 & 5.37 & .0001 \\
& B4_R & STSc_R & .72 & 9.92 & $<.0001$ \\
& PAC_R & PTr_R & .57 & 5.32 & .0001 \\
\hline
\end{tabular}

Application of a Bonferroni correction for 73 comparisons, assuming an experiment-wise alpha of $\mathrm{p}=.05$, resulting in a per comparison alpha threshold of $\mathrm{p}=.00068$. 
both left hemisphere conditions, with the theoretically critical pathway connecting the posterior with the anterior speech areas being the most troublesome. Applying this logic to the IRCs observed for the right hemisphere synonym generation data would, in agreement with the SEM results, have required the model to be rejected for the same reason, i.e. a nonsignificant correlation between areas PTr_R and STSc_R.

This not, however, to say that both approaches are of equal utility. A major advantage of the SEM approach is that, regardless of whether a model is rejected or not, SEM is able to generate an objective statistic, i.e. a chi-square statistic, that can be used to quantify model fit. Paths can then be either constrained or freed, the model rerun, and the difference in model fit, as measured by the chi-square statistic, objectively tested. As such, SEM allows us to objectively test alternative models, as long as the models tested are nested, i.e. the more constrained model can be generated by fixing one or more of the parameters comprising the less constrained model. Even if it is impossible to create nested models, thus not allowing for an objective test, one still has the option of comparing the fit of vastly divergent models via any of a number of goodness-of-fit indices. Such a comparison made it possible for us to state that the Geschwind model was a better fit to the data than the Petersen et al. model.

SEM also has the advantage of being able to test link directionality, a capability beyond that of the IRC approach. This was demonstrated in noting that, although IRC was able to show that the path linking PAC_L to B42_L became highly significant during synonym generation, the IRC approach was unable to test the hypothesis the this increase reflected B42_L acting upon PAC_L, rather than vice-versa. This hypothesis, was however, quite easily tested using SEM, allowing us to conclude that a reciprocal link from B42_L to PAC_L was not a reasonable explanation for the noted significant correlation.

The ability to suggest new connections not encompassed by the original models, although possible via both approaches, also benefits from the fact that SEM is sensitive to link directionality. For example, in examining the IRCs found in Table 8, one could note the existence of a highly significant negative correlation between PAC_L and B44_L, however, only using the information provided by the SEM modification indices could one 
note (1) the direction of the suggested path, from PAC_L to B44_L, and (2) the minimum drop in the chi-square statistic if one were to add this path, thus providing information about the significance of making the suggested change. Obviously, the addition of any new paths are subject to neuroanatomical validation. In this particular case, a direct link seems improbable, however, an indirect link might have some potential. Galaburda and Pandya (1983) noted that information can flow from PAC to multi-modal areas located at the STS and the insula; in addition, Deacon (1992) found fibers linking BA 44 with dorsal insular cortex. Such an indirect path, linking PAC to BA 44, could be tested by creating and sampling an insular cortex ROI, and then including it into the model.

Similarly, the findings of Galaburda and Pandya (1983) can also be used to give neuroanatomical validation for the inclusion of a path projecting from PAC_R to STSc_R. The addition of this path, as suggested by examination of the modification indices for the right hemisphere repetition data, would result in a significant chi-square decrease, $\mathrm{p}<.005$.

Left vs. Right Hemisphere A somewhat unexpected result of the model comparisons that were done, was that, regardless of a approach used, the best fit of data to model was achieved when fitting repetition data to the Geschwind model using right hemisphere homologous ROIs. This is particularly disturbing, since the suggestion that language is, after all, primarily a right hemisphere function, is not really worth seriously entertaining. This is not to say, however, that the right hemisphere plays no role in language processing. As a matter of fact, the finding that the right hemisphere fits the repetition data well, while not fitting the synonym generation data, is in harmony with the current view that the right hemisphere may well be quite capable of performing simple language processing, while being unable to accomplish higher level semantic tasks. The problem lies in the fact that all models were rejected when attempting to fit the left hemisphere data. As such, one feels compelled to offer possible reasons for this unexpected finding.

A couple of possibilities present themselves. Firstly, one could argue that the stronger right-sided interregional correlations might reflect the fact that the right hemisphere uses higher-level cortex to accomplish language-related processing, that may be accomplished by subcortical structures in the left hemisphere. As such, since the 
Geschwind model does not postulate the involvement of any subcortical structures, the right hemisphere data might be a better fit to the model. This hypothesis could be tested by testing various models of language processing that include subcortical components (Alexander et al., 1987; Crosson, 1985; 1992; Wallesch \& Papagno, 1988), and noting whether hemispheric differences exist with regard to model fit.

A second possibility is based upon the assumption that association cortex that is specialized for a given function might be able to accomplish a given function more efficiently, thus allowing for the implementation of the function with less tissue, whereas association cortex that is less specialized, while still being able to carry out the function, may be less efficient in how the function is implemented, and thus require more tissue. In this instance, one could argue that the language components that we are attempting to measure via our ROIs, might have a smaller spatial extent on the left than on the right. As a result, the greater spatial extent of the right hemisphere components, when considered over all subjects in the study, are more likely to overlap, resulting in higher correlations in the right hemisphere. This possibility can be tested by increasing the size of the sampling sphere, so that it is more likely to include the non-overlapping left hemisphere components. Such an increase should result in increased left hemisphere correlations.

Finally, the low left hemisphere correlations could be the result of greater interindividual variability, with regard to component localization, within the left hemisphere. This hypothesis could be examined either (1) by increasing the size of the sampling sphere, or (2) by examining the normalized blood flow peaks, across subjects, within a given radius of each ROI center, and noting differences in peak variability between hemispheres.

\section{Conclusion}

The purpose of this paper was to examine whether new PET analysis techniques could be applied in order to test the validity of models of language processing. To this end, results were obtained that bear on both the techniques used to test these models, and on the models themselves. 
With regard to modeling techniques, this study differentiates itself from all others in the way that regions of interest were assigned to processing stages. Previous studies have defined their ROIs either anatomically or physiologically. Anatomically defined templates, while benefiting from being defined a priori, have the disadvantages of being time consuming to implement, and not particularly precise with regard to spatial localization, if one is to sample the entire brain while keeping the number of ROIs manageable. Physiological ROI creation has the advantage of being more spatially precise, however, this method suffers from the fact that (1) one must define the ROIs by inspection of the current dataset, i.e. ROIs are not defined a priori, or (2) ROIs are defined by selecting a coordinate generated from single, particular study, thus limiting generalizability of that ROI to other datasets. This study, successfully, was able to steer a middle course by creating a priori defined physiological ROIs, resulting from a metaanalysis of the language activation literature. Support for the validity of the resulting ROIs was given by noting that (1) objectively created clusters were formed at regions of cortex, in accord with current knowledge about language substrates, e.g. distinct leftlateralized clusters were identified separately for BA 44 and BA 45, (2) processing that could reasonably be assumed to be bilateral, created bilateral clusters, e.g. B42_L and PTr_R, that were close in both proximity and task profile, and (3) left-sided clusters contained a significant number of data points per cluster. The major drawback to this approach is the immense overhead associated with carrying out such a meta-analysis. This drawback, however, may in the near future be overcome, as researchers develop shared activation databases, that will allow extraction of standardized coordinates for all studies within a given domain, e.g. language, or even within a given task, e.g. synonym generation. Such databases should allow researchers to quickly and easily cluster hundreds of related data points, resulting in the creation of ROIs with enough data points per ROI to compute meaningful measures of ROI dispersion. This, in turn, should allow for the creation of variable sized ROIs, rather than having to fix the ROI size, as was done in the current study.

This study also served to demonstrate that, of both of the techniques that were evaluated, the structural equation modeling technique was able to offer significantly more 
than that offered by the use of interregional correlations. It is of particular interest to note that the SEM technique does appear to be capable of modeling higher level cognitive systems, although some difficulties must still be addressed. For example, the inability of either SEM or IRCs to fit either of the models to left hemisphere data, served to point to potential inter-hemispheric differences in the organization of the language substrates, even at the level of simple word repetition. It was also of interest to note that even though the Geschwind model was able to fit the right hemisphere repetition data, it was remarkably unsuccessful at fitting the right hemisphere synonym generation data. This finding lends support to the asertion that lexico-semantic tasks may be predominantly left-lateralized.

On a technical point, it must be realized that the assignment of residuals during the modeling process via a rule of thumb method, should be recognized as a source of possible problems. Although fixing residuals at either higher or lower values will not influence the generated path coefficients, it does, however, influence the amount of variance that must be accounted for by the model, and may thereby result in either an increase or decrease in associated error terms. As such, seemingly minor adjustments in residuals may result in a given model or path being either rejected as being an unacceptable fit, or not. All that one can do to address this problem is to be aware of its existence, and perhaps explore various potential residuals settings in order to see how these adjustments might impact model fit.

A final finding of the current study, addresses the primary research question under consideration, i.e. which model fits the data best. As stated previously, the best fit to the repetition data is obtained by applying the Geschwind model to the right hemisphere data. With regard to the left hemisphere data, although neither model proved to be a particularly good fit, once again, examination of the goodness-of-fit indices point to the Geschwind model as being the least objectionable. The model of Petersen et al. was found to be a stunningly poor fit to the left hemisphere data, and could not be tested on the right hemisphere due to the lack of right-sided homologous ROIs. Also of note is that the postulated semantic association area, while not contributing significantly to fitting the synonym generation data, did make a significant contribution to the fit of the repetition 
data. A major reason for the extraordinarily bad fit of the Petersen et al. model might well be the result of postulating a direct connection between PAC_L and B39_L. The insertion of intermediate areas, such as B42_L and PT_L, might result in a much better fit of the model, since it seems as if the insertion of a particularly bad link into a model may have the effect of propagating that resulting error throughout the entire model.

\section{Future Directions}

A number of avenues still remain available with regard to using SEM to model language processes. Of greatest significance is that the problems encountered in attempting to map models onto left hemisphere data be identified and solved. The most promising and easily implemented solution involves sampling the left hemisphere using larger sampling spheres. The correction of this problem, will then allow us to refit the models and perhaps to suggest an alternative to both models. Also, the rather positive results that were obtained for the right hemisphere repetition data leads us to believe that any future model of low level language processing must include inter-hemispheric paths. Further, in an effort to develop new models to be validated with subsequent datasets, datasets could be analyzed for the existence of task-specific networks via techniques such as partial least squares (PLS) and the use of reference voxel-based techniques.

More generally, although the area of modeling language via SEM appears to be quite promising, it would seem as if the greatest strides are to be achieved by the combination of PET and SEM with other technologies. For example, although SEM can provide some information with regard to causality via the imposition of an underlying anatomical model, meaningful temporal information can best be gained by incorporating other techniques such as $\mathrm{fMRI}$ and ERPs into a test battery. Also, techniques such as transcranial magnetic stimulation can be used at the level of the individual to test whether postulated language networks are valid at the level of specific individuals, and if not, how these networks may change between individuals. The ability to make such determinations could have a profound impact in the areas of clinical neurology, neuropsychology, and aphasiology. 


\section{References}

Alexander, M. P., Benson, D. F., \& Stuss, D. T. (1989). Frontal lobes and language. Brain and Language, 37, 656-691.

Alexander, M. P., Naeser, M. A., \& Palumbo, C. L. (1987). Correlations of subcortical CT lesion sites and aphasia profiles. Brain, 110, 961-991.

Arbuckle, J. (1992). AMOS 3.1 Documentation package. Philadelphia, Penn.: Temple University.

Azari, N. P., Rapoport, S. I., Salemo, J. A., Grady, C. L., Gonzalez-Aviles, A., Schapiro, M. B., \& Horwitz, B. (1992). Interregional correlations of resting cerebral metabolism in old and young women. Brain Research, 589(2), 279-290.

Benson, D. F., \& Geschwind, N. (1969). The alexias. In P. J. Vinken and G. W. Bruyn (Eds.), Handbook of Clinical Neurology, Volume 4 (pp. 112-140). Amsterdam: NorthHolland.

Bentler, P.M. (1985). Theory and implementation of EQS, A structured equations program. Los Angeles: BMDP Statistical Software.

Binder, J. R., Rao, S. M., Hammeke, T. A., Yetkin, F. Z., Wong, E. C., Mueller, W. M., Morris, G. L. \& Hyde, J. S. (1993). Functional magnetic resonance imaging (FMRI) of auditory semantic processing. Neurology (Suppl. 2), 43, 189.

Binder, J. R., Rao, S. M., Hammeke, T. A., Frost, J. A., Bandettini, P. A., \& Hyde, J. S. (19??). Effects of stimulus rate on signal response during functional magnetic resonance imaging of auditory cortex. Cognitive Brain Research.

Binder, J. R., Rao, S. M., Hammeke, T. A., Yetkin, F. Z., Jesmanowicz, A., Bandettini, P. A., Wong, E. C., Estkowski, L. D., Goldstein, M. D., Haughton, V. M., \& Hyde, J. S. (1994a). Functional magnetic resonance imaging of human auditory cortex. Annals of Neurology, 35, 662-672.

Binder, J. R., \& Rao, S. M. (1994b). Human brain mapping with functional magnetic resonance imaging. In Andrew Kertesz (Ed.), Localization and Neuroimaging in Neuropsychology (pp. 185-212). San Diego: Academic Press. 
Bogen, J. E. \& Bogen, G. M. (1976). Wernicke's region - where is it? Annals of the New York Academy of Sciences, 280, 834-843.

Broca, P. (1861). Perte de la parole. Ramollissement chronique et destruction partielle du lobe antérieur gauche du cerveau. Bulletin de la Société d'Anthropologie, 2, 235.

Broca, P. (1865). Sur la faculté du langage articulé. Bulletin de la Société d'Anthropologie, 6, 337-393.

Brodmann, K. (1909). Vergleichende Lokalisationslehre der Grosshirnrinde in ihren Prinzipien dargestellt auf Grund des Zellenbaues. Leipzig: Barth.

Caplan, D., Gow, D., \& Makris, N. (1995). Analysis of lesions by MRI in stroke patients with acoustic-phonetic processing deficits. Neurology, 45, 293-298.

Caramazza, A. (1984). The logic of neuropsychological research and the problem of patient classification in aphasia. Brain and Language, 21, 9-20.

Carpenter, M., Sutin, J. (1983). Human Neuroanatomy. Baltimore: Williams \& Wilkins, 680-684.

Chavis, D. A., \& Pandya, D. N. (1976). Further observation on corticofrontal connections in the rhesus monkey. Brain Research, 117, 369-386.

Cipolloni, P. B., \& Pandya, D. N. (1989). Connectional analysis of the ipsilateral and contralateral afferent neurons of the superior temporal region in the rhesus monkey. The Journal of Comparative Neurology, 281, 567-585.

Crosson, B. (1985). Subcortical functions in language: A working model. Brain and Language, 25, 257-292.

Crosson, B. (1992). Subcortical functions in language and memory. New York: The Guilford Press.

Deacon, T. W. (1992). Cortical connections of the inferior arcuate sulcus cortex in the macaque brain. Brain Research, $\underline{573}$, 8-26.

Dejerine, J. (1891). Sur un cas de cécité verbale avec agraphie, suivi d'autopsie. Comptes Rendus Hebdomadaires des Séances et Mémoires de la Société de Biologie, Ninth series, $\underline{3}, 197-201$. 
Demonet, J-F., Chollet, F., Ramsay, S., Cardebat, D., Nespoulous, J-L., Wise, R., Rascol, A., \& Frackowiak, R. (i992). The anatomy of phonological and semantic processing in normal subjects. Brain, $\underline{115}, 1753-1768$.

Demonet, J-F., Wise, R., \& Frackowiak, R. S. J. (1993). Involvement of the right angular gyrus in semantic processes and of the left supra-marginal gyrus in phonological processes: An activation study using PET in normal subjects. Joumal of Cerebral Blood Fow and Metabolism, 13(suppl. 1), S547.

Demonet, J. F., Price, C., Wise, R., \& Frackowiak, R. S. (1994). Differential activation of right and left posterior sylvian regions by semantic and phonological tasks: a positron-emission tomography study in normal human subjects. Neuroscience Letters, $182,25-28$.

Desmond, J. E., Sum, J. M., Wagner, A. D., Demb, J. B., Shear, P. K., Glover, G. H., Gabrieli, J. D. E., \& Morrell, M. J. (1995). Functional MRI measurement of language lateralization in Wada-tested patients. Brain, $\underline{118}, 1411-1419$.

Drevets, W. C., Videen, T. O., MacLeod, A. K., Haller, J. W. \& Raichle, M. E. (1992). PET images of blood flow changes during anxiety: Correction. Science, 256, 1696. Efron (1981). Nonparametric estimates of standard error: the jackknife, the bootstrap, and other methods. Biometrika, $\underline{68}, 589-599$.

Ellis, A. W., \& Young, A. W. (1988). Human Cognitive Neuropsychology. Hillsdale, NJ: Lawrence Erlbaum.

Evans, A. C., Thompson, C. J., Marrett, S., Meyer, E., \& Mazza, M. (1991a). Performance evaluation of the PC-2048: a new 15-slice encoded-crystal PET scanner for neurological studies. IEEE Trans. Med. Imaging, 10, 90-98.

Evans, A. C., Marrett, S., Torrescorzo, J., Ku, S., \& Collins, L. (1991b). MRI-PET correlation in three dimensions using a volume-of-interest (VOD) atlas. Journal of Cerebral Blood Flow and Metabolism, 11, A69-A78.

Evans, A. C., Marrett, S., Neelin, P., Collins, L., Worsley, K., Dai, W., Milot, S., Meyer, E., \& Bub, D. (1992b). Anatomical mapping of functional activation in stereotactic coordinate space. Neuroimage, $1,43-53$. 
Evans, A. C., Collins, D. L., \& Milner, B. (1992a). An MRI-based brain atlas from 300 young normal subjects. Society for Neuroscience Abstracts, $\underline{18}, 408$.

Fair, C. M. (1992). Cortical memory functions. Boston: Birkhauser.

Fiez, J. A., Tallal, P., Miezin, F. M., Dobmeyer, S., Raichle, M. E., \& Petersen, S. E. (1992). PET studies of auditory processing: Passive presentation and active detection. Society for Neuroscience Abstracts, 18, 932.

Fiez, J. A., Raichle, M. E., Tallal, P. A., \& Petersen, S. E. (1993). Activation of a left frontal area near Broca's area during auditory detection and phonological access tasks. Journal of Cerebral Blood Flow and Metabolism, 13, S504.

Fiez, J. A., Raichle, M. E., Balota, D. A., Tallal, P., \& Petersen, S. E. (1996a). PET activation of posterior temporal regions during auditory word presentation and verb generation. Cerebral Cortex, $\underline{6}, 1-10$.

Fiez, J. A., Raife, E. A., Balota, D. A., Schwarz, J. P., Raichle, M. E., \& Petersen, S. E. (1996b). A positron emission tomography study of the short-term maintenance of verbal information. The Journal of Neuroscience, 16, 808-822.

Flechsig, P. (1920). Anatomie des menschlichen Gehirns und Rückenmarks. Leipzig: Georg Thieme.

Fletcher, P. C., Frith, C. D., Grasby, P. M., Shallice, T., Frackowiak, R. S. J., \& Dolan, R. J. (1995). Brain systems for encoding and retrieval of auditory-verbal memory. Brain, $118,401-416$.

Foundas, A. L., Leonard, C. M., Gilmore, R. L., Fennell, E. B., \& Heilman, K. M. (1996). Pars triangularis asymmetry and language dominance. Proceedings of the National Academy of Science of the USA, 93, 719-722.

Fox, P.T., Mintun, M.A., Reiman, E.M., \& Raichle, M.E. (1988). Enhanced detection of focal brain responses using intersubject averaging and change-distribution analysis of subtracted PET images. Joumal of Cerebral Blood Flow and Metabolism, $\underline{8}, 642$ 653.

Fox PT. (1991). Physiological ROI definition by image subtraction. [Review]. Journal of Cerebral Blood Flow \& Metabolism, 11(2), A79-82. 
Friston, K. J., Frith, C. D., Liddle, P. F., \& Frackowiak, R. S. J. (1993). Functional connectivity: The principal-component analysis of large (PET) data sets. Journal of Cerebral Blood Flow and Metabolism, 13, 5-14

Friston, K. J., Frith, C. D., Liddle, P. F., \& Frackowiak, R. S. J. (1991). Comparing functional (PET) images: the assessment of significant change. Journal of Cerebral Blood Flow and Metabolism, 11, 690-699.

Friston, K. J., Liddle, P. F., Frith, C. D., Hirsch, SR., Frackowiak, R. S. (1992a). The left medial temporal region and schizophrenia. A PET study. Brain, 115 (Pt 2), 367-82.

Frith, C. D., Friston, K., Liddle, P. F., Frackowiak, R. S. J. (1991a). A PET study of word finding. Neuropsychologia, 29, 1137-1148.

Frith, C. D., Friston, K., Liddle, P. F., Frackowiak, R. S. J. (1991b). Willed action and the prefrontal cortex in man: a study with PET. Proc. R. Soc. London Ser. B, 244, 241-246.

Galaburda, A. M. (1980). La région de Broca: Observations anatomiques faites un siècle après la mort de son découvrer. Revue Neurologiques (Paris), 136, 609-616.

Galaburda, A. M. \& Pandya, D. N. (1983). The intrinsic architectonic and connectional organization of the superior temporal region of the rhesus monkey. Journal of Comparative Neurology, 221, 169-184.

Galaburda, A., \& Sanides, F. (1980). Cytoarchitectonic organization of the human auditory cortex. Journal of Comparative Neurology, 190, 597-610.

Geschwind, N. (1965). Disconnection syndromes in animals and man. Part I-II. Brain, 88, 273-294, 585-644.

Geschwind, N. (1969). Problems in the anatomical understanding of aphasias. In A. L. Benton (Ed.), Contributions to Clinical Neuropsychology (pp. 107-128). Chicago: Aldine Publishing Co.

Geschwind, N., \& Levitsky, W. (1968). Human brain: left-right asymmetries in temporal speech region. Science, 161, 186-187.

Gold, M., Adair, J. C., Jacobs, D. H., \& Heilman, K. M. (1995). Right-left confusion in Gerstmann's syndrome: a model of body centered spatial orientation. Cortex, $\underline{31}$, 267283. 
Goldman-Rakic, P. S., Selemon, L. D., \& Schwartz, M. L. (1984). Dual pathways connecting the dorsolateral prefrontal cortex with the hippocampal formation and parahippocampal cortex in the rhesus monkey. Neuroscience, 12, 719-743.

Goldman-Rakic, P. S. (1987). Circuitry of primate prefrontal cortex and regulation of behavior by representational knowledge. Handb. Physiol., $\underline{5}$, 374-417.

Goldman-Rakic, P. S. (1988). Topography of cognition: parallel distributed networks in primate association cortex. Annual Review of Neuroscience, 11, 137-156.

Hart, J. \& Gordon, B. (1990). Delineation of single-word semantic comprehension deficits in aphasia, with anatomical correlation. Annals of Neurology, 27, 226-231.

Hayes, T. L., \& Lewis, D. A. (1995). Anatomical specialization of the anterior motor speech area: hemispheric differences in magnopyramidal neurons. Brain and Language, $\underline{49}, 289-308$.

Henderson, V. W. (1986). Anatomy of posterior pathways in reading: A reassessment. Brain and Language, 29, 119-133.

Horwitz, B., Duara, R., \& Rapoport, S. I. (1984). Intercorrelations of glucose metabolic rates between brain regions: Application to healthy males in a state of reduced sensory input. Journal of Cerebral Blood Flow and Metabolism, 4, 484-499.

Horwitz, B., Duara, R., \& Rapoport, S. I. (1986). Age differences in intercorrelations between regional cerebral metabolic rates for glucose. Annals of Neurology, 19, 6067.

Horwitz, B., \& McIntosh, A. R. (1993). Network modeling and functional neuroimaging for mapping brain cognitive function. Ann. Nucl. Med., 7, S90-S91.

Horwitz, B., Rumsey, J., Grady, C., \& Rapoport, S. I. (1988). The cerebral metabolic landscape in autism: Intercorrelations of regional glucose utilization. Arch. Neurol., $\underline{45}, 749-755$.

Horwitz, B., Soncrant, T.T., \& Haxby, J.V. (1992). Covariance analysis of functional interactions in the brain using metabolic and blood flow data. In F. Gonzalez-Lima, et al. (Eds.), Advances in metabolic mapping techniques for brain imaging of behavioral and learning functions, pp. 189-217. Netherlands. 
Howard, D., Patterson, K., Wise, R., Brown, W. D., Friston, K., Weiller, C., \&

Frackowiak, R. (1992). The cortical localization of the lexicons. Brain, $\underline{115}, 1769$ 1782.

Jöreskog, K.G., \& Sörbom, D. (1984). LISREL-VI User's Reference Guide (3rd ed.).

Mooresville IN: Scientific Software Inc.

Jöreskog, K.G., \& Sörbom, D. (1989). LISREL-7 User's Reference Guide. Mooresville

IN: Scientific Software Inc.

Kapur, S., Rose, R., Liddle, P. F., Zipursky, R. B., Brown, G. M., Stuss, D., Houle, S., \& Tulving, E. (1994). The role of the left prefrontal cortex in verbal processing: semantic processing or willed action? Neuroreport, $\underline{5}, 2193-2196$.

Klein, D., Milner, B., Zatorre, R. J., Meyer, E., \& Evans, A. C. (1995). The neural substrates underlying word generation: A bilingual functional-imaging study. Proceedings of the National Academy of Science, USA, 92, 2899-2903.

Klein, D., Zatorre, R.J., Milner, B., Johnsrude, I.S., Nikelski, J., Meyer, E., \& Evans, A.C. (1996). CBF patterns during synonym generation: group vs. individual study. In A.W. Toga, R.S.J. Frackowiak, and J. C. Mazziotta (Eds.), Neuroimage, 3, S444. Boston: Academic Press.

Lichtheim, O. (1884). On aphasia. Brain, $\underline{7}, 443-484$.

McIntosh, A. R., \& Gonzalez-Lima, F. (1992). The application of structural modeling to metabolic mapping of functional neural systems. In: NATO ASI series: Advances in metabolic mapping techniques for brain imaging of behavioral and learning functions (Gonzalez-Lima F, Finkenstaedt T, Scheich H, eds), pp 219-255. Dordrecht: Kluwer Academic.

McIntosh, A. R., \& Gonzalez-Lima, F. (1993). Network analysis of functional auditory pathways mapped with fluorodeoxyglucose: associative effects of a tone conditioned as a Pavlovian excitor or inhibitor. Brain Research, $\underline{627}$, 129-140.

McIntosh, A. R., \& Gonzalez-Lima, F. (1994). Structural equation modeling and its application to network analysis in functional brain imaging. Human Brain Mapping, 2, 2-22. 
McIntosh, A. R., Grady, C. L., Ungerleider, L. G., Haxby, J. V., Rapoport, S. I., \& Horwitz, B. (1994). Network analysis of cortical visual pathways mapped with PET. The Journal of Neuroscience, 14(2), 655-666.

Menard, M. T., Kosslyn, S. M., Thompson, W. L., Alpert, N. M., \& Rauch, S. L. (1996). Encoding words and pictures: A positron emission tomography study. Neuropsychologia, 34, 185-194.

Mesulam, M-M. (1990). Large-scale neurocognitive networks and distributed processing for attention, language, and memory. Annals of Neurology, 28, 597-613.

Mohr, J. P. (1976). Broca's area and Broca's aphasia. In H. Whitaker and N. A. Whitaker (Eds.), Studies in Neurolinguistics (pp. 201-235). New York: Academic Press Muri, R. M., Iba-Zizen, M. T., Derosier, C., Cabanis, E. A., Pierrot-Deseilligny, C. (1996). Location of the human posterior eye field with functional magnetic resonance imaging. Journal of Neurology, Neurosurgery \& Psychiatry, 60, 445-448.

Musiek, F. E., \& Reeves, A. G. (1990). Asymmetries of the auditory areas of the cerebrum. Journal of the American Academy of Audiology, 1, 240-245.

Ojemann, G. A., Fried. I., Lettich, E. (1989). Electrocorticographic (ECoG) correlates of language. I. Desynchronization in temporal language cortex during object naming. Electroencephalogr Clin Neurophysiol, 73, 453-463.

Pandya, D. N., Rosene, D. L., \& Doolittle, A. M. (1994). Corticothalamic connections of auditory-related areas of the temporal lobe in the rhesus monkey. The Joumal of Comparative Neurology, $\underline{345}, 447-471$.

Pandya, D. N., \& Sanides, F. (1973). Architectonic parcellation of the temporal operculum in rhesus monkey and its projection pattern. Zeitschrift fuir Anatomie und Entwicklungsgeschichte, 139, 127-161.

Pandya, D. N., Seltzer, B., \& Barbas, H. (1988). Input-output organization of the primate cerebral cortex. In Horst D. Steklis and J. Erwin (Eds.), Comparative Primate Biology, Volume 4, (pp. 39-80). New York: Alan R. Liss.

Pandya, D. N., Van Hoesen, G. W., \& Mesulam, M. M. (1981). Efferent connections of the cingulate gyrus in the rhesus monkey. Experimental Brain Research, 42, 319-330. 
Pandya, D. N., \& Yeterian, E. H. (1990). Prefrontal cortex in relation to other cortical areas in rhesus monkey: Architecture and connections. In H. B. M. Uylings, C. G. Van Eden, J. P. C. De Bruin, M. A. Corner, \& M. G. P. Feenstra (Eds.), Progress in Brain Research, Vol. 85 (pp. 63-94. Amsterdam: Elsevier Science Publishers B. V.

Patterson, K., Graham, N., \& Hodges, J. R. (1994). The impact of semantic memory loss on phonological representations. Journal of Cognitive Neuroscience, $\underline{6}(1), 57-69$.

Paulesu, E., Frith, C. D., \& Frackowiak, R. S. J. (1993). The neural correlates of the verbal component of working memory. Nature, $362,342-345$.

Paulesu, E., Frith, U., Snowling, M., Gallagher, A., Morton, J., Frackowiak, R. S. J., \& Frith, C. D. (1996). Is developmental dyslexia a disconnection syndrome? Evidence from PET scanning. Brain, 119, 143-157.

Paus, T., Perry., D. W., Zatorre, R. J., Worsley, K. J., \& Evans, A. C. (in press). Modulation of cerebral blood-flow in human auditory cortex during speech: role of motor-to-sensory discharges. European Joumal of Neuroscience.

Penfield, W., \& Roberts, L. (1959). Speech and Brain Mechanisms. Princeton, NJ: Princeton University Press.

Penhune, V. B., Zatorre, R. J., MacDonald, J. D., \& Evans, A. C. (1996). Interhemispheric anatomical differences in human primary auditory cortex: Probabilistic mapping and volume measurement from magnetic resonance scans. Cerebral Cortex, 6(5), 661-672.

Petersen, S. E., Fox, P. T., Posner, M. I., Mintun, M., \& Raichle, M. E. (1988). Positron emission tomographic studies of the cortical anatomy of single-word processing. Nature, $331,585-589$.

Petersen, S. E., Fox, P. T., Posner, M. I., Raichle, M. E. \& Mintun, M. (1989). Positron emission tomographic studies of the processing of single words. Journal of Cognitive Neuroscience, $1,153-170$.

Petersen, S. E., Fox, P. T., Snyder, A. Z., \& Raichle, M. E. (1990). Activation of extrastriate and frontal cortical areas by visual words and word-like stimuli. Science, 249, 1041-1044. 
Petrides, M., \& Pandya, D. N. (1988). Association fiber pathways to the frontal cortex from the superior temporal region in the Rhesus monkey. The Journal of Comparative Neurloogy, 273, 52-66.

Petrides, M., \& Pandya, D. N. (1994). Comparative architectonic analysis of the human and the macaque frontal cortex. In F. Boller and J. Grafman (Eds.), Handbook of Neuropsychology, Volume 9 (pp. 17-58). Amsterdam: Elsevier Science B.V.

Posner, M. I., \& Petersen, S. E. (1990). The attention system of the human brain. Annual Review of Neuroscience, $13,25-42$.

Posner, M. I., \& Snyder, C. R. R. (1975). Attention and cognitive control. In R. Solso (Ed.), Information Processing and cognition: The Loyola Symposium. Hillsdale, NJ: LEA.

Posner, M. I., Petersen, S. E., Fox, P. T., \& Raichle, M. E. (1988). Localization of cognitive operations in the human brain. Science, 240, 1627-1631.

Price, C. J., Wise, R. J. S., \& Frackowiak, R. S. J. (1996). Demonstrating the implicit processing of visually presented words and pseudowords. Cerebral Cortex, $\underline{6}, 62-70$.

Prieto, J. J., Peterson, B. A., \& Winer, J. A. (1994). Morphology and spatial distribution of GABAergic neurons in cat primary auditory cortex (AI). The Journal of Comparative Neurology, 344, 349-382.

Rademacher, J., Caviness, V.S., Steinmetz, H., \& Galaburda, A. M. (1993). Topographical variation of the human primary cortices: Implications for neuroimaging, brain mapping, and neurobiology. Cerebral Cortex, 3 , 313-329.

Raichle, M. E., Fiez, J., Videen, T. O., MacLeod, A. K., Pardo, J. V., Fox, P. T., \& Petersen, S. E. (1994). Practice-related changes in human brain functional anatomy during nonmotor learning. Cerebral Cortex, 4, 8-26.

Raichle, M. E., Martin, W. R. W., Herscovitch, P., Mintun, M. A., Markham, J. (1983). Brain blood flow measured with intravenous $\mathrm{H}_{2} \mathrm{O}^{15}$. II. Implementation and validation. Journal of Nuclear Medicine, 24, 790-798.

Rasmussen, T., \& Milner, B. (1975). Clinical and surgical studies of the cerebral speech areas in man. In K. J. Zulch, O. Creutzfeldt, \& G. C. Galbraith (Eds.), Cerebral Localization (pp. 238-257). New York: Springer-Verlag. 
Rasmussen, T., \& Milner, B. (1977). The role of early left-brain injury in determining lateralization of cerebral speech functions. Annals of the New York Academy of Sciences, 299, 355-369.

Rueckert, L., Appollonio, I., Grafman, J., Jezzard, P., Johnson, R. Jr., Le Bihan, D., \& Turner, R. (1994). Magnetic resonance imaging functional activation of left frontal cortex during covert word production. Journal of Neuroimaging, 4, 67-70.

Schaeffler, L., Lueders, H. O., \& Beck G. J. (1996). Quantitative comparison of language deficits produced by extraoperative electrical stimulation of Broca's, Wernicke's, and basal temporal language areas. Epilepsia, 37, 463-475.

Scheibel, A. B. (1984). A dendritic correlate of human speech. In N. Geschwind and A. M. Galaburda (Eds.), Cerebral Dominance: The Biological Foundations (pp. 43-52). Cambridge (Ma): Harvard University Press.

Schwartz, M. F. (1984). What the classical aphasia categories can't do for us, and why. Brain and Language, 21, 3-8.

Seltzer, B., \& Pandya, D. N. (1978). Afferent cortical connections and architectonics of the superior temporal sulcus and surrounding cortex in the rhesus monkey. Brain Research, 149, 1-24.

Shallice, T. (1989). From Neuropsychology to Mental Structure. Cambridge: Cambridge University Press.

Steinmetz, H., \& Seitz, R. J. (1991). Functional anatomy of language processing: neuroimaging and the problem of individual variability. Neuropsychologia, $29,1149-$ 1161.

Swinney, D., Zurif, E. (1995). Syntactic processing in aphasia. Brain \& Language, $\underline{50}$, 225-239.

Talairach, J., \& Tournoux, P. (1988). A Co-planar Stereotaxic Atlas of the Human Brain. Stuttgart: Thieme.

Vogt, B. A., Nimchinsky, E. A., Vogt, L. J., Hof, P. R. (1995). Human cingulate cortex: surface features, flat maps, and cytoarchitecture. Journal of Comparative Neurology, 359, 490-506. 
von Economo, C., \& Koskinas, G. N. (1925). Die Cytoarchitektonik der Hirnrinde des erwachsenen Menschen. Wien: Springer.

Wallesch, C. W., \& Papagno, C. (1988). Subcortical aphasia. In F. C. Rose, R. Whurr, \& M. A. Wyke (Eds.), Aphasia (pp. 256-287). London: Whurr Publishers.

Warburton, E., Wise, R. J. S., Price, C. J., Weiller, C., hadar, U., Ramsay, S., \&

Frackowiak, R. S. J. (1996). Noun and verb retrieval by normal subjects: Studies with PET. Brain, 119, 159-179.

Wernicke, C. (1874). Der aphasische Syptomencomplex. Breslau: Cohn \& Weigert.

Westbury, C., Zatorre, R. J., Evans, A. C. \& Klein, D. (1996). The planum temporale: A re-assessment of its boundaries, area, \& volume using 3D in-vivo morphometric techniques. Society for Neuroscience Abstracts, $\underline{3}, 1858$.

Williams, S. M., \& Goldman-Rakic, P. S. (1993). Characterization of the dopaminergic innervation of the primate frontal cortex using a dopamine-specific antibody. Cerebral Cortex, $\underline{3}$, 199-222.

Wise, R., Chollet, F., Hadar, U., Friston, K., Hoffner, E., \& Frackowiak, R. (1991).

Distribution of cortical neural networks involved in word comprehension and word retrieval. Brain, 114, 1803-1817.

Worsley, K. J., Evans, A. C., Marrett, S., \& Neelin, P. (1992). A three-dimensional statistical analysis for CBF activation studies in human brain. Journal of Cerebral Blood Flow and Metabolism, 12, 900-918.

Zatorre, R. J., Evans, A. C., Meyer, E. \& Gjedde, A. (1992). Lateralization of phonetic and pitch discrimination in speech processing. Science, 256, 846-849.

Zatorre. R. J., Meyer, E., Gjedde, A., \& Evans, A. C. (1996). PET studies of phonetic processing of speech: Review, replication, and reanalysis. Cerebral Cortex, $\underline{6}, 21-30$.

Zeki, S., Watson, J. D., Lueck, C. J., Friston, K. J., Kennard, C., Frackowiak, R. S. (1991). A direct demonstration of functional specialization in human visual cortex. Journal of Neuroscience, 11(3), 641-9.

Zurif, E., Swinney, D., Prather, P., Solomon, J., \& Bushell, C. (1993). An on-line analysis of syntactic processing in Broca's and Wernicke's aphasia. Brain \& Language, $\underline{45}, 448-464$. 
Zurif, E., Swinney, D., Prather, P., \& Love, T. (1994). Functional localization in the brain with respect to syntactic processing. Journal of Psycholinguistic Research, $\underline{23}$, 487-497. 


\section{Appendix A}

\section{Significance testing and the correlation coefficient}

When performing interregional correlational analyses, one is concerned with being able to judge the significance of two types of comparisons. Firstly, one is concerned with being able to detect whether a given ROI correlation pair can be said to differ significantly from zero, i.e. to be able to state that, within a given probability, these two areas appear to be correlated in the population. Secondly, when either the magnitude or the direction of a correlation between two ROIs varies from one condition to the other, one needs to be able to ascertain whether the size of the difference between coefficients is above that that one would normally expect, i.e. whether the size of the difference reflects a different underlying population correlation. How these two types of differences can be tested, is detailed below.

\section{Testing the difference between a correlation coefficient and zero}

We shall first consider testing for a significant difference between a given correlation coefficient and zero. The test is quite simple and is given in equation (1), as presented by Howell (1992).

$$
t=\frac{r-\rho}{s_{r}}
$$

Since we are testing whether our correlation coefficient is significantly different from a population value of zero, the value of rho in the equation can be set to zero. The Pearson's $r$ is calculated in the usual way. The remaining term in the equation, $s_{r}$ ,representing the standard error associated with the correlation, can be calculated as shown in equation (2).

$$
s_{r}=\sqrt{\frac{1-r^{2}}{N-2}}
$$

We would now, thus, be in a position to generate a t-value, distributed on $\mathrm{N}-2$ degrees of freedom. Unfortunately, this is not quite possible, because the estimate of standard error given in equation (2) assumes $\mathrm{N}$ to be quite large, and therefore as $\mathrm{N}$ decreases in size, the estimate of the standard error becomes less and less valid. A 
solution to this problem inyolves using the sample itself to generate an estimate of the standard error, and will be discussed shortly.

\section{Testing the difference between two correlation coefficients}

Tests for a significant difference between two correlation coefficients use a z-score rather than the t-statistic used in the previous section (Howell, 1992). The computation to generate the $z$-score is given in equation (3).

$$
z=\frac{r_{1}-r_{2}}{\sqrt{s_{r_{1}}+s_{r_{2}}}}
$$

The numerator of the calculation contains the two correlation coefficients, that have been transformed via the Fisher transform in order to make the distribution of $\underline{\mathbf{r}}$ approximately normal. The computation for the Fisher transform is shown in equation (4).

$$
r^{\prime}=(0.5) \log _{e}\left|\frac{1+r}{1-r}\right|
$$

The denominator of equation (3) contains the estimates of standard error for both correlations. As one might suspect, these estimates are also unstable when computed on a small sample size. However, for completeness, the standard computation used to estimate standard error is given in equation (5).

$$
s_{r}=\frac{1}{\sqrt{N-3}}
$$

\section{Estimation of standard error}

The previous two sections attempted to make clear that the estimation of standard error for correlation coefficients is profoundly effected by sample size. Horwitz et al. (1992) addressed this problem by using a bootstrap procedure (Efron, 1981) to estimate the standard error based on the actual sample itself. The bootstrap procedure works as follows: (1) given a sample of size $\mathrm{N}$, randomly select, with replacement, a subsample, also of size $\mathrm{N},(2)$ calculate the correlation coefficient for the subsample and apply the Fisher transform, (3) perform steps (1) and (2) X number of times, and (4) the standard 
error for the correlation is given by computing the standard deviation of the $\mathrm{Xr}^{\prime}$ values. We are now free to enter the bootstrapped standard error into equations (1) or (3).

The only question that remains to be addressed is with regard to how many subsamples (X) should be taken during the bootstrap procedure. Horwitz et al. (1992) state that they were able to obtain stable results with 100 subsamples, when applying this method to regional metabolic data. Since it was unknown whether this recommended value was particular to metabolic data, or perhaps influenced by the size of the sample used by Horwitz et al., it was decided to perform a test in order to confirm the recommended use of 100 subsamples. The test was carried out by performing bootstraps, using numbers of subsamples varying from 10 to 500, selected from the repetition rCBF data. Figure A-1, which is comprised of the correlations that were found to be the most labile across the number of subsamples used, illustrates the results of the test. Of note are: (1) all correlations show a great deal of instability within the 10-60 subsamples range, and (2) estimates of standard error do not stabilize until we reach the 200 subsamples point. In particular, it should be noted that a number of estimates shift dramatically between 100 and 200 subsamples. All estimates appear to be quite stable for all subsamples greater than 200. As such, it was decided to use the bootstrapped estimates based upon 200 subsamples, rather than the 100 figure suggested by Horwitz et al. Since the reasons for the discrepancy between the findings of Horwitz et al. and the current study are unclear, it would seem wise for researchers to perform similar tests when attempting to arrive at an optimal value for the number of subsamples. 

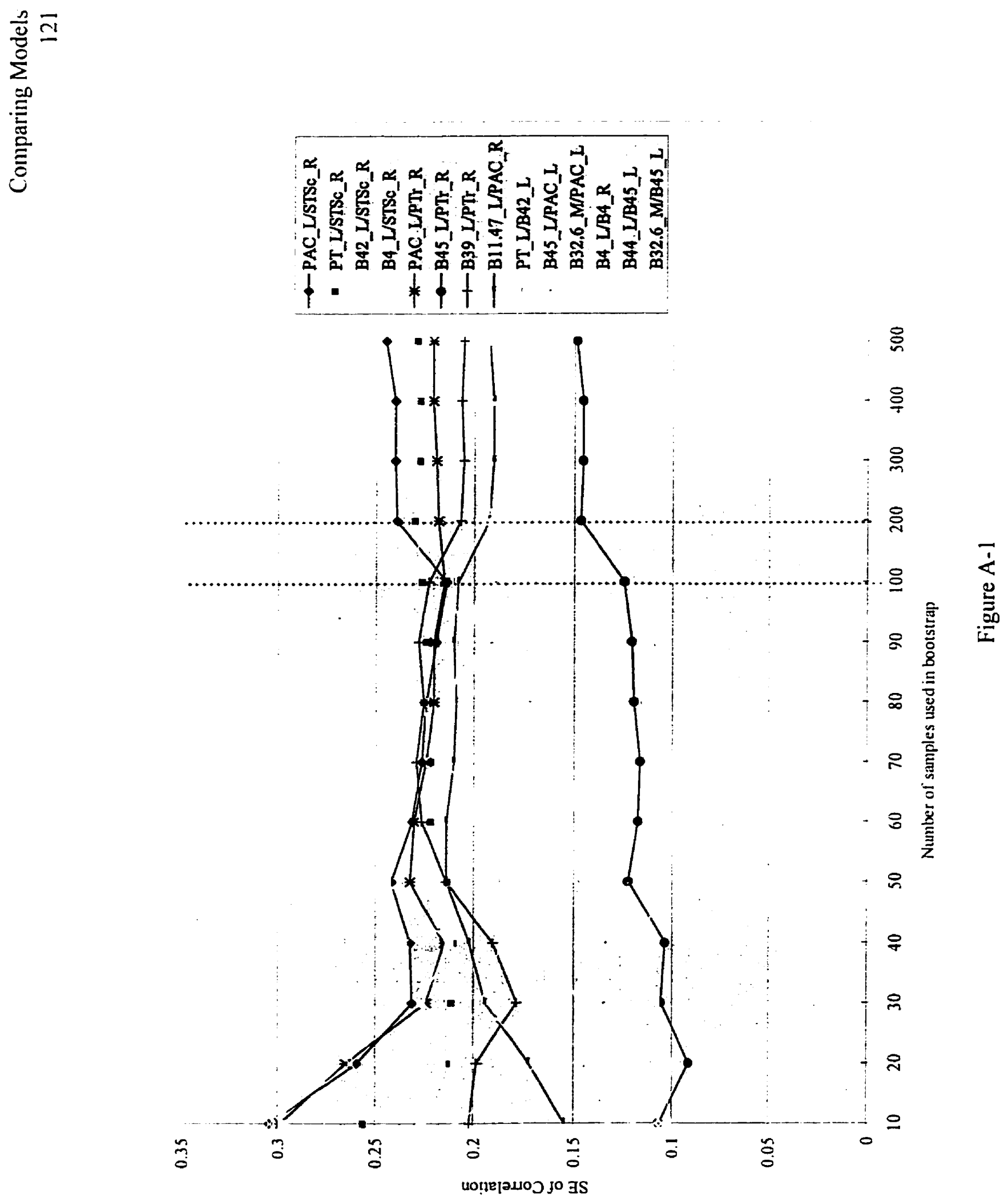
IMAGE EVALUATION

TEST TARGET (QA-3)
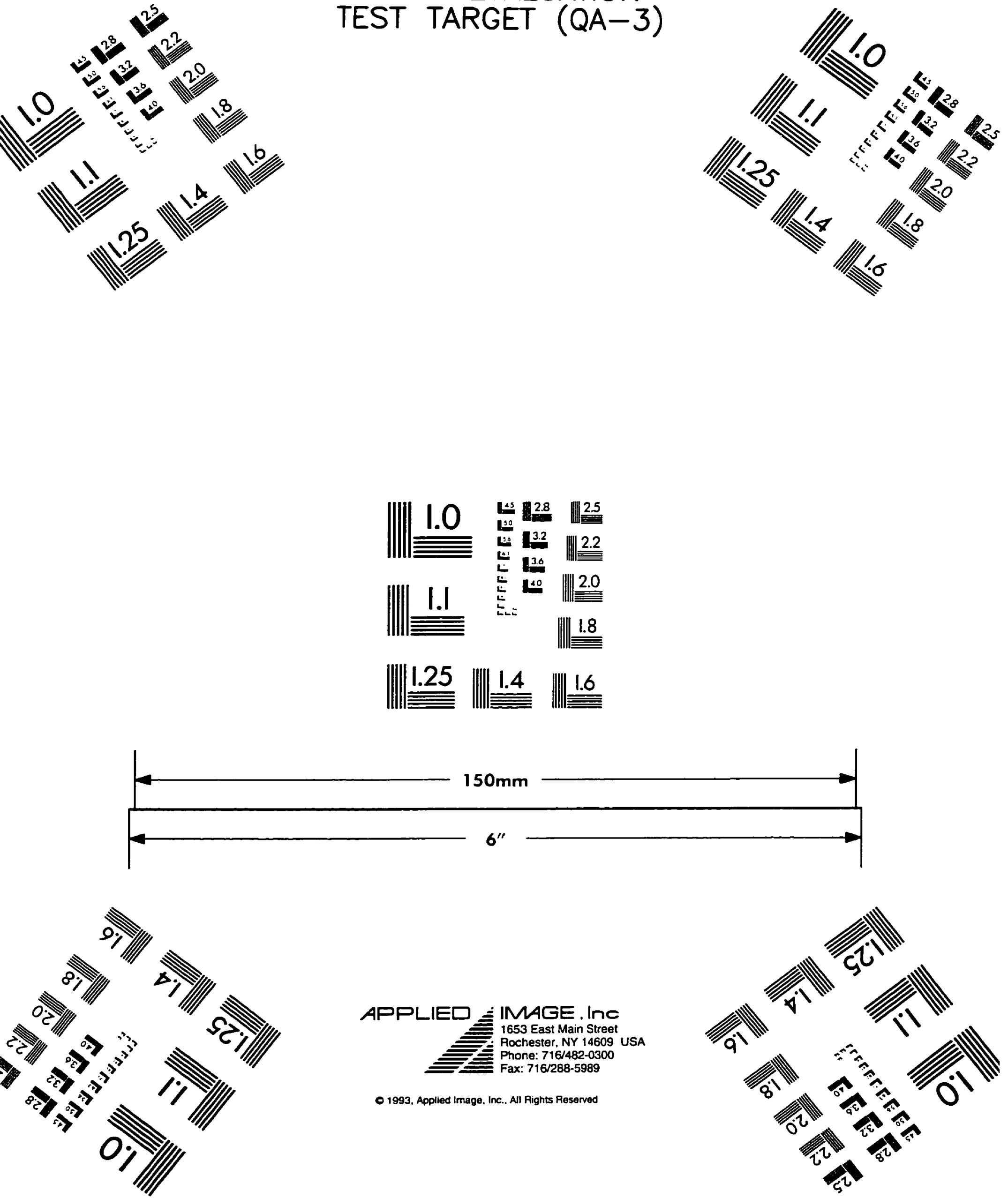